\title{
Reaction of Initially Distant Scalars in a Cylinder Wake by
}

\section{Tanaya R. Kawakami}

A.S., Western Nevada College, 2007

B.S., University of Nevada, Reno, 2010

\author{
A thesis submitted to the \\ Faculty of the Graduate School of the \\ University of Colorado in partial fulfillment \\ of the requirements for the degree of
}

Master of Science

Department of Civil, Environmental, \& Architectural Engineering 
This thesis entitled:

Reaction of Initially Distant Scalars in a Cylinder Wake written by Tanaya R. Kawakami

has been approved for the Department of Civil, Environmental, \& Architectural Engineering

John P. Crimaldi

Roseanna M. Neupauer

Harihar Rajaram

Date

The final copy of this thesis has been examined by the signatories, and we find that both the content and the form meet acceptable presentation standards of scholarly work in the above mentioned discipline. 
Kawakami, Tanaya R. (M.S., Civil Engineering)

Reaction of Initially Distant Scalars in a Cylinder Wake

Thesis directed by Associate Professor John P. Crimaldi

We used analytical and numerical techniques to examine reaction rates of two initially distant scalars released into a cylinder wake at a Reynolds number of 97.4. Two scalars, $C_{1}$ and $C_{2}$, are released into the flow upstream of the cylinder with an initial lateral separation. As the scalars advect in the flow, the plumes coalesce in the well-developed vortex street where the plumes are stretched and folded by vortex interaction. The scalars diffuse along the elongated interface and mix together, or react.

The finite element analysis software, COMSOL, is used to solve the Navier-Stokes equations for the cylinder wake flow field, and a pair of coupled advection-diffusion-reaction equations are solved for the transport and reaction of two initially distant scalar concentrations. In a series of simulations, we examine the reaction rates for various scalar release geometries, reaction speeds, and scalar diffusivities. For each model, the reaction rate is calculated by lateral integrating the $C_{1} C_{2}$ product and phase averaging over one shedding period.

A model without the cylinder is created as a baseline case for direct comparisons to the cylinder wake case. With no stirring mechanism in the flow, scalar reaction only occurs by diffusion. Comparing the cylinder wake case with the no-cylinder case shows the effect on reaction due directly to the wake.

The analytical solution is derived for the limiting case of integrated reaction rate and is found to be a function of a single parameter. The analytical solution, numerically integrated solution, and the numerical simulation show good agreement, validating the numerical models.

The cylinder wake cases showed significant increase in reaction rate compared to the nocylinder cases for all parameters tested, particularly at large separations, in which, the cylinder wake increased the reaction rate an order of magnitude. 


\section{Dedication}

I dedicate this thesis to my sisters

Kyra Blaize and Chase Meridian 


\section{Acknowledgements}

This accomplishment would not have been possible without the support and encouragement of others. I would like to give special thanks to those who have shown me endless patience, taught me more than just fluid mechanics, and have been by my side throughout this journey.

Professor John Crimaldi - I wish to express to you my deepest appreciation and gratitude. Without your support and guidance none of this would have been possible.

My Committee - Thank you for teaching me to never accept less than excellence, and for your kindness and patience. Your teaching is truly an inspiration, both in the classroom and in life. Each of you have had a great influence on me. Thank you for preparing me, professionally and academically, for a successful career in an exciting field.

Crimaldi Research Group and Office Mates - Thank you all for your support and friendships.

Allison Bell - Thank you for always being here to listen and "talk it through" with me.

Mike Soltys - Thank you for your generous assistance with coding and sharing key concepts in new ways.

My family - Thank you for encouraging me to do my best and teaching me that if something is worth doing, it is worth doing well; to take pride in everything I do and have no regrets.

Andrew Bell - Thank you for being my biggest supporter. Your love, support, and encouragement has brought me though some of the toughest times in this journey. 


\section{Contents}

\section{Chapter}

1 Introduction 2

1.1 Problem Description . . . . . . . . . . . . . . . . . . . . . 2

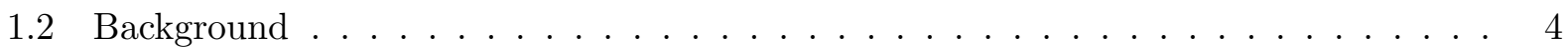

1.2 .1 Cylinder Wakes . . . . . . . . . . . . . . . . . . . . 4

1.2 .2 Vortex Mixing and Reaction . . . . . . . . . . . . . . . 8

1.3 Brief Thesis Outline . . . . . . . . . . . . . . . . . 10

2 Analytical Analysis 11

2.1 Navier-Stokes . . . . . . . . . . . . . . . . . . . . . . . . 12

2.2 Advection Diffusion Reaction . . . . . . . . . . . . . . . . . . 12

2.2 .1 Concentration Equations . . . . . . . . . . . . . . . 13

2.2 .2 Reaction Equation . . . . . . . . . . . . . . . . . 18

2.3 Dimensional Analysis . . . . . . . . . . . . . . . . . . . . . . . . . . 19

3 Numerical Model and Postprocessing 22

3.1 Numerical Model . . . . . . . . . . . . . . . . . . . . . . . . 22

3.1 .1 Cylinder Model . . . . . . . . . . . . . . . . . . . . 22

$3.1 .2 \quad$ No-Cylinder Model . . . . . . . . . . . . . . . . . . . 33

3.2 Postprocessing Analyisis . . . . . . . . . . . . . . . . 35 
4 Reactions Without Cylinder Wake $\quad 36$

4.1 Limiting Reaction Rate Maximum . . . . . . . . . . . . . 36

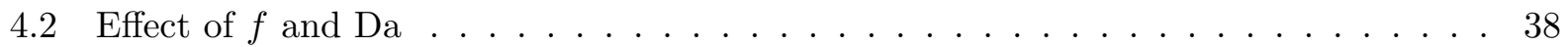

5 Reactions With Cylinder Wake 42

5.1 Effect of Scalar Separation . . . . . . . . . . . . . . . . . 43

5.2 Effect of Filament Width . . . . . . . . . . . . . . . . . . 45

5.3 Effect of Schmidt Number . . . . . . . . . . . . . . . . . . . . 47

5.4 Effect of Damköhler Number . . . . . . . . . . . . . . . . . . . . . . . 49

5.5 Effect of Cylinder Wake . . . . . . . . . . . . . . . . . . 51

$5.5 .1 \quad$ Exception Case . . . . . . . . . . . . . . . . . . . 55

5.5 .2 Total Product . . . . . . . . . . . . . . . . . 57

6 Conclusions $\quad 60$

6.1 No-Cylinder Case . . . . . . . . . . . . . . . . . . . . . . . 60

6.2 Cylinder Case . . . . . . . . . . . . . . . . . . . . . . . 61

6.3 Future Work . . . . . . . . . . . . . . . . . . . 62

6.3 .1 Discrete Release . . . . . . . . . . . . . . . . . . . . . 62

6.3 .2 Asymmetrical Release . . . . . . . . . . . . . . . . . . . . . 62

6.3 .3 Asymmetrical Investigation $1 \ldots \ldots . \ldots \ldots$

6.3 .4 Asymmetrical Investigation $2 \ldots \ldots \ldots \ldots \ldots$

$\begin{array}{ll}\text { Bibliography } & 67\end{array}$

\section{Appendix}

A Model Parameters 
$\begin{array}{ll}\text { B MATLAB Code } & 75\end{array}$

B.1 Numerical Integration Solution $($ Eqn. 2.21) $\ldots \ldots \ldots \ldots$

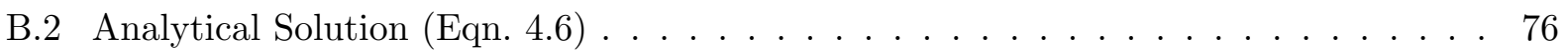

B.3 Laterally integrated, phased averaged reaction rate processed from data exported

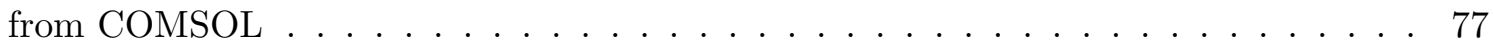

C Proof for integral identity Eqn. 4.5 


\section{Tables}

\section{Table}

A.1 Model Variables . . . . . . . . . . . . . . . . . . 73

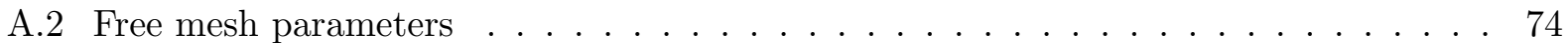




\section{Figures}

\section{Figure}

2.1 Release of two scalars, $C_{1}$ and $C_{2}$, into a left-to-right flow. Scalars are released with a small lateral separation. . . . . . . . . . . . . . . . . . . . 11

2.2 Diffusion evolution of two scalars, $C_{1}$ and $C_{2}$, initially in contact with each other, shown as the concentration profiles at three locations. . . . . . . . . . 16

2.3 Scalar concentration profiles at three downstream locations showing diffusion evolution for the $C_{1}$ and $C_{2}$ scalars with separation, $s$, and filament width, $f \ldots \ldots 17$

2.4 $C_{1}$ and $C_{2}$ scalar profiles at some downstream location and the associated reaction

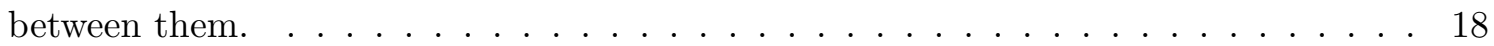

3.1 Geometry and dimensions of the model where the origin of the coordinate system is at the cylinder center. . . . . . . . . . . . . . . . . 23

3.2 Model mesh grid generated using Comsol free mesher. Regions 1 and 3 have very refined mesh and regions 2 and 4 have a coarse mesh. . . . . . . . . . . . . . 24

3.3 (a) Velocity streamlines for the flow field, and (b) velocity streamlines shown with most the mean velocity in the streamwise direction removed revealing the vortex

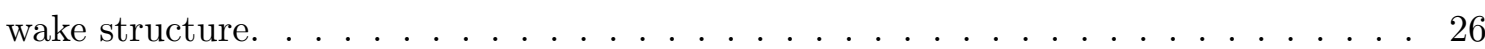

3.4 Time history of the velocity at a point in the cylinder wake shown for three consecutive 30 -second simulations overlaid to show convergence. . . . . . . . . . . . . 27

3.5 Scalar release geometry and dimensions for two scalar concentrations, $C_{1}$ and $C_{2}$, released into the flow with scalar separation, $s$, and filament width, $f . \ldots . \ldots 28$ 
3.6 Virtual and actual scalar release locations in model domain. . . . . . . . . . . . . . 29

3.7 Concentration profiles at virtual and actual scalar release locations. . . . . . . . . 30

3.8 (a)Instantaneous $C_{1}$ and $C_{2}$ scalars at quasi-steady-state. Scalars highlight the welldeveloped vortex street, and (b)associated reaction rate. . . . . . . . . 31

3.9 Reaction concentration profiles along the centerline for models with varying number of total mesh elements. . . . . . . . . . . . . . . . . . . . . 32

3.10 Reaction concentration profiles along the centerline for models with varying number of total mesh elements. . . . . . . . . . . . . . . . . . . 32

3.11 (a) Scalar concentrations $C_{1}$ and $C_{2}$ and (b) $R / k$ reaction images for the no-cylinder model at steady-state. . . . . . . . . . . . . . . . . . . . 33

$3.12 C_{1}$ and $C_{2}$ scalar transport model at steady-state with three locations marked. Concentration profiles at these location are shown in Fig. $3.13 \ldots \ldots$. . . . . . 34

$3.13 C_{1}$ and $C_{2}$ scalar concentration profiles of Comsol data and analytical solution for three $x$-locations. The agreement between the analytical solution and the numerical simulation validates the model. $\ldots \ldots \ldots \ldots \ldots \ldots$

4.1 Analytical solution for reaction rate as a function of $\lambda$ on (a) linear scale, and (b) $\log -\log$ scale, showing the limiting case for the maximum reaction rate. . . . . . . . 37

4.2 Integrated reaction rate for the no-cylinder case for $f^{*}=14.7,2.94$, and 1.47 , where, the analytical solution is the solid line, the dashed lines are the numerically integrated solution, and the symbols are the simulation results. . . . . . . . . . . 39

4.3 Integrated reaction rate results for the no-cylinder case for $\mathrm{Da}=0.0018,0.18$, and 1.8, where, the analytical solution is the solid line and the symbols are the simulation

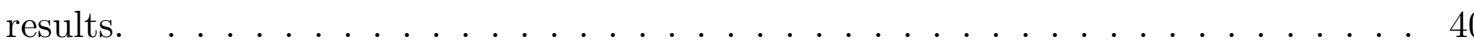

5.1 Instantaneous scalar concentrations, $C_{1}$ and $C_{2}$ (left column) and associated reaction rate (right column) for large separation, $s^{*}=2$ (top row), and small separation,

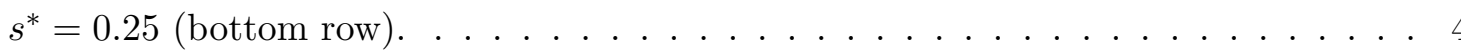


5.2 Color scale for all scalar concentration fields (left) and logarithmic color scale for all reaction rate images $($ right $) \ldots \ldots \ldots \ldots \ldots \ldots \ldots \ldots$

5.3 Integrated reaction rates for cylinder wake simulations of various scalar separations, where $s^{*}=0,0.25,1,2$ and $f^{*}=3, \mathrm{Sc}=10$, and $\mathrm{Da}=0.01$, plotted with downstream distance scaled by cylinder diameter. . . . . . . . . . . . . . . . 45

5.4 Instantaneous scalar concentrations, $C_{1}$ and $C_{2}$ (left column) and associated reaction rate (right column) for the smaller $f^{*}=0.25$ (top row) and larger $f^{*}=3$ (bottom row)

5.5 Integrated reaction rates of cylinder wake simulations for various scalar filament widths, where $f^{*}=0.25,0.5,1,3$, and $s^{*}=0.25, \mathrm{Sc}=10$, and $\mathrm{Da}=0.01 . \ldots 47$

5.6 Instantaneous scalar concentrations, $C_{1}$ and $C_{2}$ (left column) and associated reaction rate (right column) for $\mathrm{Sc}=1$ (top row) and $\mathrm{Sc}=20$ (bottom row) . . . . . . . 48

5.7 Integrated reaction rates for cylinder wake simulations of $\mathrm{Sc}=1,10,20$ with constant $s^{*}=0.25, f^{*}=3$, and $\mathrm{Da}=0.01$ plotted with downstream distance scaled by cylinder diameter. . . . . . . . . . . . . . . . . . . . . . . 49

5.8 Integrated reaction rates for cylinder wake simulations of $\mathrm{Da}=0.01,10,100$ with constant $s^{*}=0.25, f^{*}=3$, and $\mathrm{Sc}=10$, plotted with downstream distance scaled by cylinder diameter. . . . . . . . . . . . . . . . . . 50

5.9 Integrated reaction rates for the cylinder wake case and the no-cylinder case for (a) $s^{*}=0.25$ and (b) $s^{*}=1$, while $f^{*}=3, \mathrm{Sc}=10$, and $\mathrm{Da}=0.01$, plotted with downstream distance scaled by cylinder diameter. . . . . . . . . . . . . . . . . 51

5.10 Integrated reaction rate results for cylinder models with various values of $s / \phi$, compared to the analytical solution for no-cylinder wake. Broken lines are results from the cylinder model, solid line is for the no-cylinder model.

5.11 Reaction rates for the cylinder wake case and the no-cylinder case for (a) $f^{*}=1$ and (b) $f^{*}=3$, while $s^{*}=0.25, \mathrm{Sc}=10$, and $\mathrm{Da}=0.01$, plotted with downstream distance scaled by cylinder diameter. . . . . . . . . . . . . . . . 53 
5.12 Integrated reaction rate results for cylinder models with various values of Sc, compared to the analytical solution for no-cylinder wake. Broken lines are results from the cylinder model, solid line is for the no-cylinder model. . . . . . . . . . . . . 54

5.13 Reaction rates for the cylinder wake case and the no-cylinder case for (a) $\mathrm{Da}=10$ and (b) $\mathrm{Da}=100$, while $s^{*}=0.25, f^{*}=3$, and $\mathrm{Sc}=10$, plotted with downstream distance scaled by cylinder diameter. . . . . . . . . . . . . . . 55

5.14 Instantaneous scalar concentrations, $C_{1}$ and $C_{2}$ (left column) and associated reaction rate (right column) for large release geometry of $s^{*}=2$ and $f^{*}=3$ (top row) and small release geometry of $s^{*}=0.25$ and $f^{*}=0.25$ (bottom row). . . . . . . 56

5.15 Reaction rates for the cylinder wake case and the no-cylinder case for simulations with (a) $f^{*}=0.25$ and (b) $f^{*}=0.5$ while $s^{*}=0.25, \mathrm{Sc}=10$, and Da $=0.01$, plotted with downstream distance scaled by cylinder diameter. . . . . . . . . . . . 57

5.16 Normalized $C_{1}$ flux for models with various Da values. The plot shows the $C_{1}$ mass that passes across an $x$-location per unit width out of the page per unit time. . . . 58

5.17 Total product flux for models with various values of Da plotted with downstream distance scaled by cylinder diameter. The plot shows the mass of product that crosses an $x$-location per unit width out of the page per unit time. . . . . . . . 59

6.1 Eccentricity, $e$, is the distance, in terms of cylinder diameters, from the stagnation streamline to the centerline between the scalar concentrations at the release. . . . . .

6.2 Scalar concentrations, $C_{1}$ and $C_{2}$, shown for an asymmetric release geometry (left) where $e=0.4$ cylinder diameters, and the corresponding reaction rate between the $C_{1}$ and $C_{2}$ scalar concentrations (right). $\ldots \ldots \ldots \ldots$

6.3 Laterally integrated phase averaged reaction rate for a cylinder model with small release geometry, comparing the symmetrical and asymmetrical release geometries,

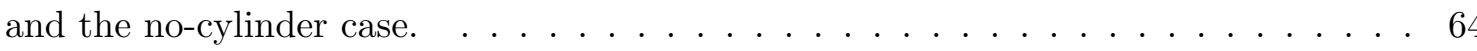


6.4 Scalar concentrations, $C_{1}$ and $C_{2}$, shown for an asymmetric release geometry (left) where $e=0.4$ cylinder diameters and the corresponding reaction rate between the

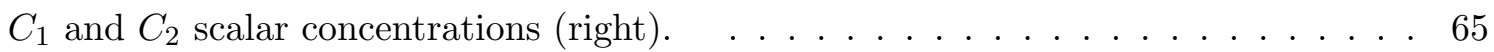

6.5 Laterally integrated phase averaged reaction rate for a cylinder model with $s^{*}=0.25$ and $f^{*}=3$, comparing the symmetrical and asymmetrical release geometries, and

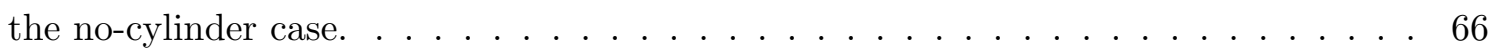




\section{Nomenclature}

\begin{tabular}{|c|c|c|c|}
\hline $\operatorname{Re}$ & Reynolds number & $R^{*}$ & non-dim. reaction rate \\
\hline St & Strouhal number & $f$ & filament width \\
\hline$f_{q}$ & vortex shedding frequency & $\lambda$ & $\frac{\text { lateral dist. have diffused }}{\text { lateral dist. need to diffuse }}$ \\
\hline$\phi$ & cylinder diameter & $x_{s}^{*}$ & non-dim. (by $s) x$ \\
\hline $\mathbf{u}$ & velocity field & $y_{s}^{*}$ & non-dim. (by $s$ ) $y$ \\
\hline$u$ & $x$-component of velocity & $f_{s}^{*}$ & non-dim. (by $s$ ) $f$ filament width \\
\hline$v$ & $y$-component of velocity & $\mathrm{R}$ & reaction rate \\
\hline$D$ & diffusion coefficient & $T_{0}$ & shedding period \\
\hline$t$ & time & $\mathcal{R}^{*}$ & non-dim. reaction rate (integrated) \\
\hline $\mathrm{Sc}$ & Schmidt number & $y^{*}$ & non-dim. (by $\phi) y$ \\
\hline$\nu$ & kinematic viscosity & $s^{*}$ & non-dim. (by $\phi) s$ separation \\
\hline$\mu, \eta$ & dynamic viscosity & $f^{*}$ & non-dim. (by $\phi) f$ filament width \\
\hline$\rho$ & density & $\mathbf{n}$ & normal vector \\
\hline $\mathrm{Da}$ & Damköhler number & $p$ & pressure \\
\hline$l$ & length scale & $p_{0}$ & initial pressure \\
\hline$k$ & reaction rate constant & $\mathbf{t}$ & tangential vector \\
\hline$C_{0}$ & initial scalar concentration & g & gravity \\
\hline $\mathrm{Pe}$ & Péclet number & $\Delta t$ & time step \\
\hline$u_{0}$ & initial velocity & $\Delta x$ & length of mesh element \\
\hline$s$ & scalar separation & $C$ & scalar concentration \\
\hline $\mathbf{u}^{*}$ & nondimensional velocity field & $\mathbf{N}_{1}, \mathbf{N}_{2}$ & scalar flux \\
\hline$p^{*}$ & non-dim. pressure & $\mathcal{R}$ & dimensional reaction rate (integrated) \\
\hline$C^{*}$ & non-dim. scalar concentration & $\xi$ & variable of integration \\
\hline
\end{tabular}




\section{Chapter 1}

\section{Introduction}

\subsection{Problem Description}

The Kármán vortex street, developed in the wake of a cylinder, is the quintessential case of the vortex shedding phenomenon, the beauty and significance of which has been recognized not only by scientists, but artists alike[1,2,3]. Cylinder wake has been studied in the context of many different disciplines in both natural sciences $[4,5,6]$ and engineering sciences[7, 8, 9] and can span a wide range of length scales, from droplet[10] and particle[11] dispersion to flow around an ocean island $[12,13,14]$.

In aquatic ecosystems, diffusion and stirring facilitated by structured flow, such as cylinder wake, enhances population growth of the system[15] by bringing together species and nutrients[16]. Furthermore, structured flow plays a key role in the coexistence of species competing for a resource[17]. As currents advect past benthic topology, such as sea-mounts, the flow can become distorted and structured whereby, under certain conditions, the flow will be dominated by a vortex street causing mixing[18]. This mechanism can be applied to reproduction. Many benthic invertebrates reproduce by broadcast spawning, a process by which plumes of gametes are released, at separate locations, into the ambient water and must coalesce for fertilization to occur[19, 20]. In the case of corals, gametes are typically released into the flow in the presence of neighboring coral heads which act as obstacles that add structure to the flow and subsequently stir the gametes. Using broadcast spawning as motivation, the current study investigates how the laminar wake behind a circular cylinder (e.g. neighboring coral head, or other benthic topology) can promote coalescence between 
initially distant scalars (sperm and egg gametes) to facilitate reactions (gamete fertilization).

To study this problem, we use a finite element numerical model to simulate the two-dimensional laminar unsteady wake behind a smooth circular cylinder. Much is known about the flow field enabling this study to focus on the coalescence and reaction enhancement of passive reactive scalar concentrations. Using the results of the flow field simulation, we release two scalar concentrations into the flow with some initial lateral separation, upstream of the cylinder. The interaction of the vortices stretch the scalars into long filaments which are swirled and folded around the vortices. This process of stretching and folding brings filaments together that contain scalar particles that were once quite distant. Once two filaments are in close proximity of each other, the diffusing scalars react together with second order reaction kinetics. In a single vortex, stirring accelerates the coalescence of two initially separated scalars and enhances the resulting reaction rates[21]. The current study investigates if similar effects exist in the more complicated structure of a vortex street in a cylinder wake.

To elucidate the role of the cylinder wake on reaction enhancement, we run a simulation without the cylinder, however with the same model set-up and parameters as the model with the cylinder. In this case, the passive scalar concentrations advect with the undisturbed flow and diffuse laterally until the scalars are in close proximity to mix together and react. This no-cylinder simulation reaches a steady-state and is solved analytically. The simple simulation is compared to the analytical solution as a way to validate the simulated cylinder model. The analytical solution and the simulated solution are in good agreement and is one measure to show that the simulation is accurate.

In a series of simulations, several parameters are varied to investigate the subsequent effects, including different scalar release geometries, reaction rates, and scalar diffusivities. The final reaction quantities for each case are plotted against the case with no cylinder to see the increase in reaction rate. 


\subsection{Background}

\subsubsection{Cylinder Wakes}

The flow around a smooth circular cylinder in an infinite domain is controlled by a single parameter, the Reynolds number $(\operatorname{Re})$. For $5<\operatorname{Re}<40$, boundary layers separate from the cylinder and roll up in opposite directions into two symmetrical vortices that stay attached in the steady flow [22]. At all $\operatorname{Re}<40$, instabilities develop in the recirculation region[23] causing vortices to shed[22]. The instabilities cause the two vortices, which make up the recirculation region, to form asymmetrically, one growing in size and strength more quickly than the other[22]. The larger vortex, still fed by the vorticity in the free shear layer, will eventually grow strong enough to pull the weaker vortex across the center wake. The weaker vortex has opposing vorticity which quickly negates the vorticity feeding the larger vortex thus causing the larger vortex to break free and detach from the recirculation region, or formation region. The freed vortex advects down stream with the ambient flow. The former weaker vortex is now the larger vortex and continues to grow until it is strong enough to pull the other vortex across the wake, and the entire process continues and repeats to form the Kármán vortex street[24].

The formation region, also known as the chaotic region at larger Re, is the region immediately behind the cylinder where vortices are formed. The length of this region is determined by balancing the incoming free shear layer with the reversed flow circulating in the formation region[24]. The end of the formation region is the location at which the vortex has reached its maximum strength and has detached[25, 26]. At $\operatorname{Re} \sim 8000$, the formation region can be as much 2.5 cylinder diameters in length and decreases with $\operatorname{Re}[27]$. The formation region length has been found to affect the shedding frequency[24].

The flow oscillation behind a cylinder can be considered a main characteristic of bluff-body wakes, with the wake frequency being a fundamental parameter[28]. The formation region length and the diffusion width of the free shear layers are two characteristic length scales that determine the vortex shedding frequency[24]. As Re increases, the formation region decreases in length such 
that the shear layers are brought closer together, facilitating their interaction and shortening the shedding period[24]. Conversely, as Re increases, the shear layers become more diffuse (increasing the diffusion length), which then increases the time for vorticity to entrain and accumulate to a sufficient concentration, thus increasing the time to initiate shedding and lengthening the period[24]. Normalizing the vortex shedding frequency by the flow velocity and the cylinder diameter gives the Strouhal number, or the nondimensional shedding frequency, which is a function of the Reynolds number. The Strouhal number, St, can be defined as

$$
\mathrm{St}=\frac{f_{q} \phi}{u}
$$

where, $f_{q}$ is vortex shedding frequency, $\phi$ is cylinder diameter, and $u$ is velocity. The StrouhalReynolds number relationship is fairly well defined. Low Strouhal values start around 0.12 at $\operatorname{Re} \sim$ 50 and monotonically increase, asymptotically approaching $\mathrm{St}=0.21$ at $\mathrm{Re} \sim 250$. The material porosity of the cylinder has been found to affect shedding frequency.

Increasing the permeability of a cylinder will dramatically reduce the shedding frequency and the strength of the shed vortex[29]. The relative surface roughness of the cylinder has a significant impact on the Strouhal number[22] and the scale of disturbances in the water[28]. Surface roughness will transition a flow to turbulent much faster than for a smooth surface[22]. At high Reynolds number, Strouhal number increases with cylinder roughness[30]. Superhydrophobic cylinders significantly decrease drag in both laminar and turbulent flows, and the length of the recirculation region increases which delays the onset of vortex shedding[31].

The circular cylinder is the most commonly investigated cylinder, considered the quintessential bluff body[3]. The square cylinder has been found to have a significantly lower shedding frequency than the circular cylinder, while the diamond cylinder has a shedding frequency just slightly higher than the circular cylinder[28]. The angle of incidence of the square cylinder also affects the location at which the shear layers separate, which then determines the downstream flow field[32]. Other studies have investigated the range of Strouhal numbers for the triangular prism cylinder and the elliptical cylinder aligned at various angle of incidence[33] and it was found that 
the angle and the Reynolds number have a significant impact on the wake size[34].

Many studies have investigated the wake formed behind an array of cylinders. The wake behind a row of square cylinders transitions from steady to unsteady at a decreased Reynolds number as the gap ratio between the cylinders decreases creating a jet-like flow between the cylinders[35]. For $150<\operatorname{Re}<14300$, the vortex street formed by two cylinders side-by-side changed to two vortex streets[36].

Experiments on a slightly tapered cone in a uniform flow revealed spanwise cells shed regularly and periodically, though frequency changes from cell to cell spanwise along the cylinder due to a modulated motion[37]. A wavy cylinder, with various combinations of spanwise length and wave amplitude, in a laminar flow has a significant influence on the development of the free shear layer and the formation of three-dimensional vortices[38]. For $152<\operatorname{Re}<674$, a cylinder with a stepwise change in diameter was found to have a lower shedding frequency behind the larger cylinder near the step, while neighboring cells had higher frequencies[39].

The phenomenon of vortex shedding occurs when there is interaction between the two shear layers[22]. Preventing this interaction with use of a one diameter length splitter plate attached to the cylinder reduces the shedding frequency[28] since the free shear layers must advect the length of the plate before rolling up into vortices[40]. As the length of the splitter plate is increased, a secondary vortex forms around the trailing edge of the plate and the occurrence of shear layer rollup begins closer to the trailing edge, while for longer plates, the shear layer reattaches to the splitter plate[40]. An interesting alteration to the splitter plate set-up is placing the splitter plate such that there is a gap between the cylinder and the plate. A one diameter splitter plate with a variable gap between the cylinder and the splitter plate has the same effect on the wake at all Reynolds numbers examined, and, varying the gap size has a greater effect on the shedding frequency than on the vortex speed[28].

The characteristics of a cylinder wake is affected by the proximity of solid boundaries and free surfaces. A wall near a cylinder will cause the shear layers to develop asymmetrically where the shear layer on the wall side develops more weakly than the other shear layer causing the interaction 
between the shear layers to be weak[22]. A cylinder near a wall causes various other changes in the flow, including: suppressed vortex shedding for small gap-ratios, a lower angular stagnation point, a change in the separation points, and there is less suction on the wall side of the cylinder[22]. In the case of a cylinder near the free surface, a vorticity layer generates adjacent to a layer from the cylinder which, together form a jet-like flow[41].

The incoming flow upstream of a cylinder may exhibit shear in two directions: cross-stream or spanwise. In the case of cross-stream shear with small and moderate shear steepness values, vortex shedding is minimally affected, while for larger shear steepness values, shedding is significantly influenced[22, 42]. For the case of spanwise shear, vortices shed with constant frequency in spanwise cells where there is a correlation between the cellular structure and the degree of shear[22]. Increasing the steepness factor for a constant Reynolds number increases the wake width and decreases the base pressure and the Strouhal number[43]. Spanwise shear flow around a cylinder with a stepwise change in diameter was found to influence the length of the low frequency cell near the step, as well as the difference in frequency of that call with neighboring cells[39].

The Reynolds number describes the flow regime. A flow with $\operatorname{Re}<1$ is a Stokes flow in which the velocity of the fluid typically is very slow, or the length scale is very small. The shear layers do not separate as this flow passes around a cylinder. As Re increases, a wake begins to develop behind the cylinder, more quickly in the lateral direction than streamwise[44] and a pair of fixed vortices develop immediately behind the cylinder[22]. At $\operatorname{Re} \sim 40$, instabilities in the wake cause the attached vortices to alternately detach and advect downstream forming a vortex street[45]. At $\operatorname{Re} \sim 100$, the vortices begin to shed periodically[45]. Diffusion of vorticity is no longer the primary factor in determining vortex strength and frequency, and there are no standing vortices as convection begins to overwhelm diffusion near the cylinder[28]. The flow transitions to turbulent at the critical Re, $200<\operatorname{Re}<300$, and as Re increases, the formation region length decreases[27]. In the turbulent regime, $\operatorname{Re}>300$, turbulence in the incoming flow has a significant effect on the variation of Strouhal number with Reynolds number[22].

As flow transitions to turbulent, two-dimensional shedding takes on a third dimensionality as 
vortices shed in cells, which are out of phase, in the spanwise direction[22]. Shedding patterns in the third dimension have provoked inquiry while advancements in technology and computation power have allowed more thorough investigations into dimensionality with 3D models becoming more detailed. More specifically, direct numerical simulation solutions can provide an understanding into the physic of the flow field[23].

Oblique shedding occurs as vortices shed from the cylinder at some angle that can be controlled by modifying the end conditions of the cylinder to induce parallel shedding[23]. Alternatively, two upstream cylinders can be used to induce oblique, curved, or parallel shedding off of a third cylinder, from which measurements of static pressure and velocity show that oblique shedding is caused by a non-symmetric pressure distribution and the angle of oblique shedding can be predicted to with 2 degrees using the ratio of the streamwise vorticity component to the spanwise vorticity component[46].

Cylinder oscillation in the cross flow direction substantially increases the correlation length, or average length of the spanwise cells, and increases more so with an increase in the oscillation amplitude[22]. In the low Re flow, as Re increases, the rotational speed of a freely rotating cylinder decreases as two symmetrical wakes form on either side of the cylinder[47].

In general, the cylinder wake phenomenon of vortex shedding is well studied and understood. Comprehensive books describe in great detail the fundamental mechanics and equations, and other diverse aspects of cylinder flow $[22,48]$. Other articles review old and new techniques in experimental[49, 23] and modeling methods[50].

\subsubsection{Vortex Mixing and Reaction}

Early investigations identify three stages of stirring and mixing of two scalars initially in contact: the initial condition, induced motion, and homogenization[51]. From the initial condition, mixing by diffusion occurs where the random motion of molecules across the interface between the fluids slowly smooths out the non-uniformity $[52,53]$. The diffusion length scale is defined as $\sqrt{4 D t}$, where, $D$ is the diffusion coefficient, and $t$ is time. Using this relationship, it is clear that diffusion 
alone is a relatively slow process for any given length scale and, in most cases, an inadequate mixing mechanism. Consequently, there has been much interest in accelerating mixing time by stirring. Mixing is a process of homogenizing concentrations quickly and efficiently by imposing a velocity field as a mode of stirring[54], which will stretch and fold the initially distinct scalars into filaments throughout the space [55]. These stretched scalar striations exhibit a much greater concentration gradient and has the potential to significantly accelerate the onset of mixing $[51,56]$. The interface between the striated scalar filaments is lengthened so that the diffusive mixing is significantly accelerated $[53,57,58]$ which closely links the mixing rate with the interface stretching rate[59].

Mechanical mixing, or stirring, can exist as a structured flow, such as a vortex, in which, eddy motion will increase the mean gradient between two initially distinct scalars in the vortex and increase the interfacial area of the scalars while decreasing the distance between the scalars[51]. Vortex stirring accelerates molecular diffusion[58,60] by accelerating the coalescence of the scalars relative to pure diffusion and enhances the resulting reaction rates between the two initially separated reactive scalars[21, 61]. Vortex strength and the elapsed time since the vortex initiation are factors on which vortex mixing enhancement is linearly dependent; mixedness and dissipation both follow this dependence[62]. The time to mix scalars in a vortex scales as $\mathrm{Pe}^{1 / 3}[21,53,58,63]$. Near the vortex core, however, dissipation occurs more slowly, and mixing scales only as $\mathrm{Pe}^{1 / 2}[63]$.

The Schmidt number, Sc, is the ratio of kinematic viscosity, or momentum diffusion to molecular, or mass diffusion and is defined as

$$
\mathrm{Sc}=\frac{\nu}{D}=\frac{\mu}{\rho D}
$$

where, $\nu$ is kinematic viscosity, $D$ is molecular diffusivity, $\mu$ is dynamic viscosity, and $\rho$ is density. Scalars that are weakly diffusive relative to the diffusivity of momentum are characterized by a high Sc. When these weakly diffusive scalars are stirred, though the interfacial length is increased, there is little diffusion to spread and mix the scalars, resulting is less reaction. Whereas, a more diffuse scalar with a lower Sc disperses more readily increasing the reaction when provoked by stirring.

In the current study, second order reaction kinetics are used to quantify the reaction between 
two passive reactive scalar concentrations. Reaction between the two scalars is the product of the concentrations scaled by the reaction rate constant. This means that for reaction to take place, both scalars must exist at a location at the same time. The type II Damköhler number, Da, is the ratio of the diffusivity timescale to the chemical timescale. In this study, Da is defined as

$$
\mathrm{Da}=\frac{l^{2} k C_{0}}{D}
$$

where, $l$ is a length scale (either scalar separation $s$ or cylinder diameter $\phi$ ), $k$ is the reaction rate constant, and $C_{0}$ is initial concentration. The Damköhler number creates a relationship between the diffusion time scale with the reaction time scale. An increasing Da, with a constant diffusion coefficient, corresponds to an increasing reaction rate. The Péclet number, Pe, is another relevant ratio that compares the advection rate to the diffusion rate which has the form $\mathrm{Pe}=l u / D$, where, $l$ is the characteristic length, and $u$ is velocity. In this study, Pe is defines as

$$
\mathrm{Pe}=\frac{u_{0} s}{16 D}
$$

where, $u_{0}$ is initial velocity, and $s$ is scalar separation. For very small Pe, diffusion dominates, however, as Pe increases, advection dominates and the reactants can have large gradients. For the case of low Da and high Pe, reactants mixed in a vortex have a nondimensional reaction rate that grows as $\mathrm{Pe}^{1 / 3}$, while the time to the peak reaction decreases as $\mathrm{Pe}^{-2 / 3}[21]$.

\subsection{Brief Thesis Outline}

Chapter 1 gives an introductory explanation and describes the motivation of the work done in this study. Previous studies on cylinder wake, vortex mixing, and reaction are briefly reviewed as background. Chapter 2 gives the analytical analysis of the governing equations of the problem, including the Navier-Stokes equations, the advection-diffusion-reaction equations, and the dimensional analysis giving the meaningful parameters of the problem. Chapter 3 explains the set up of the numerical model and post processing techniques. Chapter 4 gives the results of the no-cylinder cases. Chapter 5 gives the results for the cylinder wake cases. Chapter 6 gives concluding remarks. 


\section{Chapter 2}

\section{Analytical Analysis}

Fluid transport occurs as some combination of advection and diffusion[64]. Transport in nature can be difficult to model due to complicated flow fields. Transport in an idealized onedimensional flow field, such as the no-cylinder model in this study, is much simpler to model, and is solved analytically. The equations that govern the transport of $C_{1}$ and $C_{2}$ in this simple model and the reaction and dimensional analysis for the problem are covered in this chapter. In Fig. 2.1, two scalars are released with a small lateral separation into a velocity field with left-to-right flow.

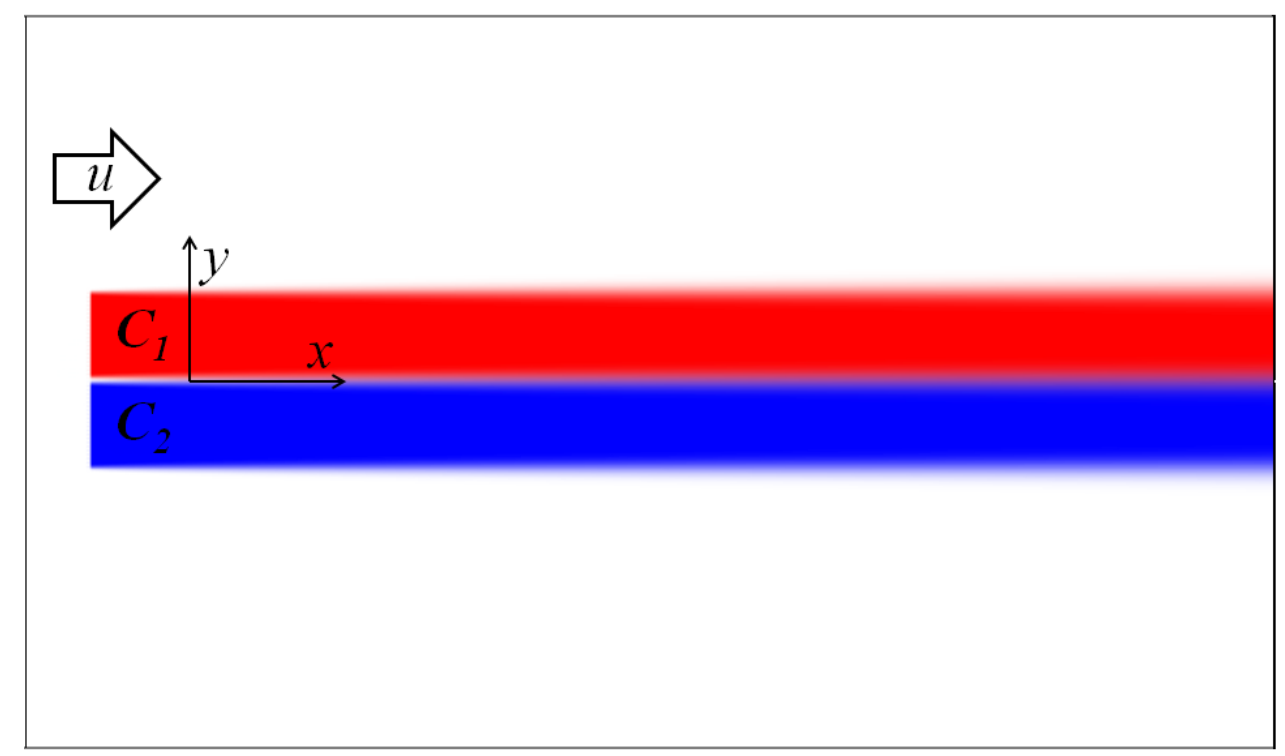

Figure 2.1: Release of two scalars, $C_{1}$ and $C_{2}$, into a left-to-right flow. Scalars are released with a small lateral separation. 


\subsection{Navier-Stokes}

The governing equations for fluid flow are the Navier-Stokes equations. For the two-dimensional cylinder flow, the equations are

$$
\begin{gathered}
\partial_{t} \mathbf{u}^{*}+\mathbf{u}^{*} \cdot \nabla \mathbf{u}^{*}=-\nabla p^{*}+\operatorname{Re}^{-1} \nabla^{2} \mathbf{u}^{*} \\
\nabla \cdot \mathbf{u}^{*}=0
\end{gathered}
$$

where, $\mathbf{u}^{*}$ is the velocity field, $p^{*}$ is pressure, and length, time, velocity, and pressure have been scaled by $\phi, \phi / u_{0}, u_{0}$, and $\rho u_{0}^{2}$, respectively. The Reynolds number, Re, is based on the cylinder diameter,

$$
\operatorname{Re}=\frac{u_{0} \phi}{\nu}
$$

where, $\phi$ is cylinder diameter, $u_{0}$ is initial velocity, $\rho$ is density, and $\nu$ is kinematic viscosity. Equation 2.1 solves momentum transport over time by balancing the local and advective accelerations with forces, such as pressure, viscosity, and gravity, and Eqn. 2.2 is the equation of continuity for incompressible fluids. The complicated unsteady cylinder wake flow field is left to be solved in the numerical simulation. The no-cylinder case has a simple one-dimensional steady-state flow field $\mathbf{u}=(1,0)$

\subsection{Advection Diffusion Reaction}

The governing equation for mass transport in a fluid is the advection-diffusion (AD) equation. For a reactive scalar mass, the transport and reaction is governed by the advection-diffusion-reaction (ADR) equation, given as

$$
16 \mathrm{Pe}\left(\partial_{t} C^{*}+\mathbf{u}^{*} \cdot \nabla C^{*}\right)=\nabla^{2} C^{*}-\mathrm{Da} R^{*}
$$

where, $C^{*}$ is scalar concentration, $\mathbf{u}^{*}$ is the velocity field, $R^{*}$ is reaction rate, and $\mathrm{Pe}$ and $\mathrm{Da}$ are the Péclet number and Damköhler number, respectively, and where velocity is still scaled by $u_{0}$, but length, time, concentration, and reaction rate are scaled by $s, s / u_{0}, C_{0}$, and $k C_{0}^{2}$, respectively, 
where $s$ is scalar separation, $u_{0}$ is initial velocity, $C_{0}=C_{1}^{0}=C_{2}^{0}$ is the initial concentration of the scalars, and $k$ is reaction rate constant. Péclet number is the ratio of the advection rate to the diffusion rate, and can be defined as

$$
\mathrm{Pe}=\frac{u_{0} s}{16 D}
$$

where, $D$ is molecular diffusivity. The Damköhler number is the ratio of the diffusive timescale to the reaction timescale, and can be defined as

$$
\mathrm{Da}=\frac{l^{2} k C_{0}}{D}
$$

where, $l$ is a length scale, either scalar separation $s$ or cylinder diameter $\phi$. The transport and reaction between two interacting scalars, $C_{1}$ and $C_{2}$, can be written as a pair of coupled ADR equations,

$$
\begin{aligned}
& 16 \operatorname{Pe}\left(\partial_{t} C_{1}^{*}+\mathbf{u}^{*} \cdot \nabla C_{1}^{*}\right)=\nabla^{2} C_{1}^{*}-\mathrm{Da} R^{*} \\
& 16 \operatorname{Pe}\left(\partial_{t} C_{2}^{*}+\mathbf{u}^{*} \cdot \nabla C_{2}^{*}\right)=\nabla^{2} C_{2}^{*}-\mathrm{Da} R^{*}
\end{aligned}
$$

where, the equations for $C_{1}$ and $C_{2}$ are coupled by the reaction,

$$
R^{*}=C_{1}^{*} C_{2}^{*}
$$

In the cylinder wake model, the complicated flow field and the coupled ADR equations are left to be solved by the numerical simulation. The model without the cylinder has a simple flow field which is used, with Eqns. 2.5 and 2.6, to solve the reaction rate for the limiting analytical case. Expressions for the concentrations and the reaction rate are developed in the follow sections.

\subsubsection{Concentration Equations}

In this study two reactive scalar concentrations, $C_{1}$ and $C_{2}$, are continuously released into a flow field, and react when they come in contact with each other. In this section, equations are derived that describe the scalar release geometry and the diffusive behavior of the scalars. The 
initial condition for the scalar concentration is

$$
C(y, t=0)=f(y)= \begin{cases}0, & y<0 \\ C_{0}, & y>0\end{cases}
$$

The general steady-state solution (where $t=x / u$ ) to the initial conditions $f(y)$ for a two-dimensional continuous source is

$$
C(x, y)=\int_{-\infty}^{\infty} \frac{f(\xi)}{\sqrt{4 \pi D x / u}} \exp \left[\frac{-(y-\xi)^{2}}{4 D x / u}\right] \mathrm{d} \xi
$$

where, $\xi$ is a variable of integration and the initial distribution is given by

$$
f(\xi)= \begin{cases}0, & \xi<0 \\ C_{0}, & \xi>0\end{cases}
$$

$$
\text { Let } U=\frac{y-\xi}{\sqrt{4 D x / u}} \text {, then } \mathrm{d} U=\frac{-1}{\sqrt{4 D x / u}} \mathrm{~d} \xi \text {, such that } \mathrm{d} \xi=-\sqrt{4 D x / u} \mathrm{~d} U
$$

Convert the limits of integration using the new variable $U$

$$
\begin{gathered}
\text { for } \xi=0, \rightarrow U=\frac{y-0}{\sqrt{4 D x / u}}=\frac{y}{\sqrt{4 D x / u}} \\
\text { for } \xi=\infty \rightarrow U=\frac{y-\infty}{\sqrt{4 D x / u}}=-\infty
\end{gathered}
$$

Substituting in $U, \mathrm{~d} U$ and the new limits of integration into the general concentration equation,

Eqn. 2.9

$$
C(x, y)=\int_{\frac{y}{\sqrt{4 D x / u}}}^{-\infty} \frac{C_{0}}{\sqrt{4 \pi D x / u}} \exp \left[-U^{2}\right] *-\sqrt{4 D x / u} \mathrm{~d} U
$$

Simplifying the expression

$$
C(x, y)=\frac{-C_{0}}{\sqrt{\pi}} \int_{\frac{y}{\sqrt{4 D x / u}}}^{-\infty} \exp \left[-U^{2}\right] \mathrm{d} U
$$

Pull out negative and switch limits

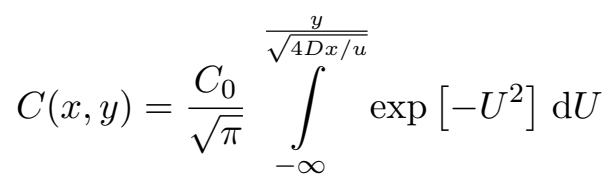


Split up integral

$$
C(x, y)=\frac{C_{0}}{\sqrt{\pi}}\left[\int_{-\infty}^{0} \exp \left[-U^{2}\right] \mathrm{d} U+\int_{0}^{\frac{y}{\sqrt{4 D x / u}}} \exp \left[-U^{2}\right] \mathrm{d} U\right]
$$

Using the Gaussian Integral, the first integral is equivalent to $\sqrt{\pi} / 2$

$$
C(x, y)=\frac{C_{0}}{\sqrt{\pi}}\left[\frac{\sqrt{\pi}}{2}+\int_{0}^{\frac{y}{\sqrt{4 D / u}}} \exp \left[-U^{2}\right] \mathrm{d} U\right]
$$

The second integral can be solved using the Gauss Error Function

$$
C(x, y)=\frac{C_{0}}{\sqrt{\pi}}\left[\frac{\sqrt{\pi}}{2}+\frac{\sqrt{\pi}}{2} \operatorname{erf}\left(\frac{y}{\sqrt{4 D x / u}}\right)\right]
$$

Simplifying the equation gives the one-sided solution for concentration diffusion in a flow

$$
C(x, y)=\frac{C_{0}}{2}\left[1+\operatorname{erf}\left(\frac{y}{\sqrt{4 D x / u}}\right)\right]
$$

Now, say there is a second scalar, $C_{2}$, arranged symmetrically about $y=0$ with the first scalar, $C_{1}$. The expressions for the diffusion of the two concentrations are

$$
\begin{aligned}
& C_{1}(x, y)=\frac{C_{1}^{0}}{2}\left[1+\operatorname{erf}\left(\frac{y}{\sqrt{4 D x / u}}\right)\right] \\
& C_{2}(x, y)=\frac{C_{2}^{0}}{2}\left[1-\operatorname{erf}\left(\frac{y}{\sqrt{4 D x / u}}\right)\right]
\end{aligned}
$$

The diffusion evolution of two scalars initially in contact with each other is shown in Fig. 2.2. The figure shows the concentration profiles at three locations. 


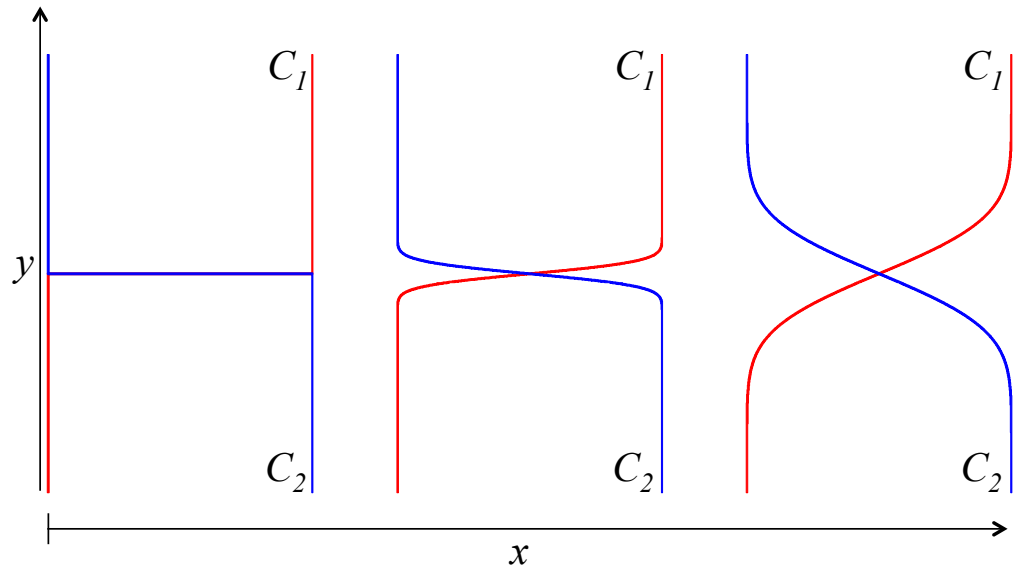

Figure 2.2: Diffusion evolution of two scalars, $C_{1}$ and $C_{2}$, initially in contact with each other, shown as the concentration profiles at three locations.

Equations 2.11 and 2.12 are for the case of two scalars initially in contact with each other, in an infinite domain. The scalars are given a finite width, $f$, and a separation distance, $s$, to define the distance between the two scalars, shown in Fig. 2.3. These dimensions are incorporated into the concentration equations and using a similar approach as before, the concentration solutions become

$$
\begin{aligned}
& C_{1}(x, y)=\frac{C_{1}^{0}}{2}\left[\operatorname{erf}\left(\frac{y-s / 2}{\sqrt{4 D x / u}}\right)-\operatorname{erf}\left(\frac{y-s / 2-f}{\sqrt{4 D x / u}}\right)\right] \\
& C_{2}(x, y)=\frac{C_{2}^{0}}{2}\left[\operatorname{erf}\left(\frac{y+s / 2+f}{\sqrt{4 D x / u}}\right)-\operatorname{erf}\left(\frac{y+s / 2}{\sqrt{4 D x / u}}\right)\right] .
\end{aligned}
$$




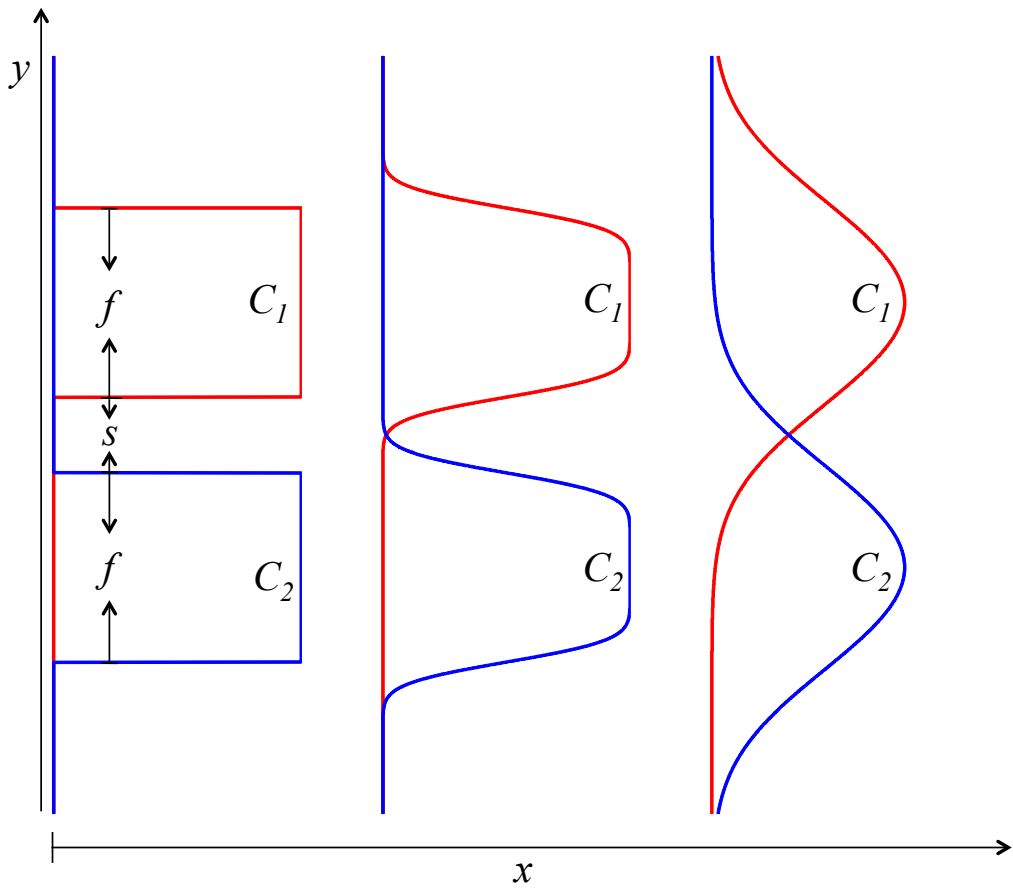

Figure 2.3: Scalar concentration profiles at three downstream locations showing diffusion evolution for the $C_{1}$ and $C_{2}$ scalars with separation, $s$, and filament width, $f$.

Equations 2.13 and 2.14 are the complete dimensional forms of the concentrations and are plotted in Fig. 2.3 showing the concentration profile at three downstream locations. The diffusion length scale in Eqns. 2.13 and 2.14, $\sqrt{4 D x / u}$, is the lateral distance the scalars have diffused at the downstream location $x$. This metric scaled by the distance each scalar must diffuse for reaction to occur, $s / 2$, can be written as the parameter $\lambda$, in terms of Pe (Eqn. 1.4),

$$
\begin{aligned}
\lambda & =\frac{\sqrt{4 D x / u_{0}}}{s / 2} \\
\lambda & =\sqrt{\frac{x_{s}^{*}}{\mathrm{Pe}}}
\end{aligned}
$$

where, $x_{s}^{*}=x / s$. The concentration equations can be nondimensionalized by the initial concentration, $C_{0}$, and written in terms of $\lambda$,

$$
\begin{aligned}
& C_{1}^{*}=\frac{C_{1}}{C_{0}}=\frac{1}{2}\left[\operatorname{erf}\left(\frac{y_{s}^{*}-1 / 2}{\lambda / 2}\right)-\operatorname{erf}\left(\frac{y_{s}^{*}-1 / 2-f_{s}^{*}}{\lambda / 2}\right)\right] \\
& C_{2}^{*}=\frac{C_{2}}{C_{0}}=\frac{1}{2}\left[\operatorname{erf}\left(\frac{y_{s}^{*}+1 / 2+f_{s}^{*}}{\lambda / 2}\right)-\operatorname{erf}\left(\frac{y_{s}^{*}+1 / 2}{\lambda / 2}\right)\right]
\end{aligned}
$$


where, $y_{s}^{*}=y / s, x_{s}^{*}=x / s$, and $f_{s}^{*}=f / s$. Equations 2.17 and 2.18 are the fully nondimensional concentration equations which are used in Chapter 4 to obtain analytical reaction results.

\subsubsection{Reaction Equation}

The reaction rate, $R$, between the two scalar, is the coupling term in the ADR equations. The reaction rate between the $C_{1}$ and $C_{2}$ scalars, can be calculated as the product of the concentrations and the reaction constant, $k$ :

$$
R(x, y)=k C_{1} C_{2}
$$

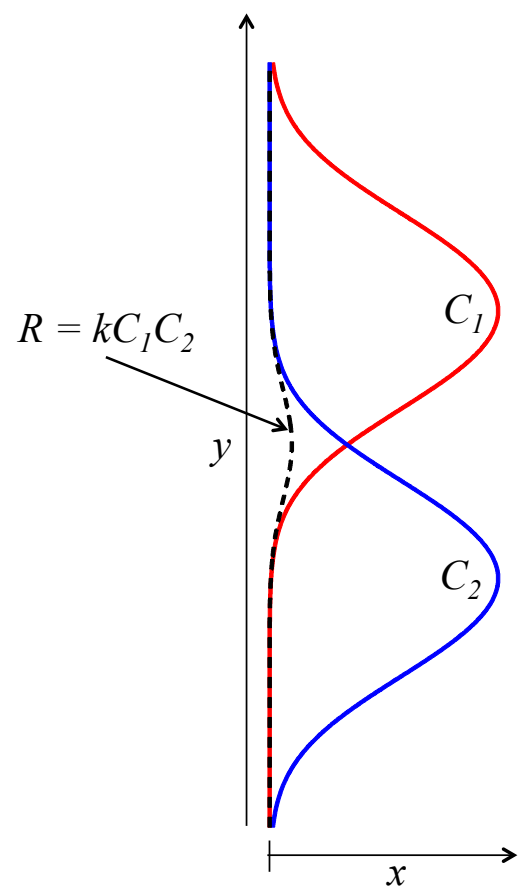

Figure 2.4: $C_{1}$ and $C_{2}$ scalar profiles at some downstream location and the associated reaction between them.

The reaction rate is scaled by the reaction rate constant, $k$, giving just the $C_{1} C_{2}$ product: $R / k=C_{1} C_{2}$. This spatial reaction is nondimensionalized by scaling the reaction by the initial 
scalar concentrations

$$
R^{*}=\frac{R / k}{C_{0}^{2}}=\frac{C_{1} C_{2}}{C_{0}^{2}}=C_{1}^{*} C_{2}^{*}
$$

where, $R^{*}$ is the nondimensional reaction rate and is a function of $x$ - and $y$-location. The expression for the total nondimensional reaction rate at a lateral downstream cross-section can be calculated by laterally integrating the reaction expression. Furthermore, the reaction rate is averaged over one shedding period, $T_{0}$, shown in Eqn. 2.21 giving the total phase-averaged reaction rate as a function of downstream distance

$$
\mathcal{R}^{*}(x)=\frac{1}{T_{0}} \int_{0}^{T_{0}} \int_{-\infty}^{\infty} R^{*} \mathrm{~d} y_{s}^{*} \mathrm{~d} t
$$

where, $y^{*}=y / s$. This nondimensional reaction rate is the metric by which all the cylinder wake models are compared. For the simple case without the cylinder, the nondimensional reaction at steady-state is similar to Eqn. 2.21 without the time averaging,

$$
\mathcal{R}^{*}(x)=\int_{-\infty}^{\infty} R^{*} \mathrm{~d} y_{s}^{*}
$$

\section{$2.3 \quad$ Dimensional Analysis}

The Buckingham-Pi method of dimensional analysis is used to produce dimensionless groups that are independent but not unique. These dimensionless groups are identified as various parameters that the model is dependent on. The relevant dimensional variables are 


\begin{tabular}{llll} 
& Symbol & Description & Units \\
\hline 1 & $\phi$ & Cylinder diameter & $\mathrm{m}$ \\
2 & $u$ & Inflow velocity & $\mathrm{m} / \mathrm{s}$ \\
3 & $R$ & Reaction rate & $\mathrm{mol} /\left(\mathrm{m}^{3} \cdot \mathrm{s}\right)$ \\
4 & $\nu$ & Kinematic viscosity & $\mathrm{m}^{2} / \mathrm{s}$ \\
5 & $D$ & Scalar diffusivity & $\mathrm{m}^{2} / \mathrm{s}$ \\
6 & $k$ & Reaction rate constant & $\mathrm{m}^{3} /(\mathrm{mol} \cdot \mathrm{s})$ \\
7 & $s$ & Scalar separation & $\mathrm{m}$ \\
8 & $f$ & Filament width & $\mathrm{m}$ \\
9 & $C_{0}$ & Initial concentration & $\mathrm{mol} / \mathrm{m}^{3}$
\end{tabular}

There are 9 dimensional variables and 3 primary dimensions: length, mass, and time. The difference, 6 , is the number of dimensionless groups that are produced. The dimensionless groups are listed and identified as

\begin{tabular}{ll} 
Parameter & Equation \\
\hline Reynolds number & $\mathrm{Re}=\frac{\phi u}{\nu}$ \\
Reaction rate & $R^{*}=\frac{R}{k C_{0}^{2}}$ \\
Damköhler number & $\mathrm{Da}=\frac{k \phi^{2} C_{0}}{D}$ \\
Schmidt number & $\mathrm{Sc}=\frac{\nu}{D}$ \\
Filament separation & $s^{*}=\frac{s}{\phi}$ \\
Filament width & $f^{*}=\frac{f}{\phi}$
\end{tabular}

Six dimensionless parameters are identified as significant to the model. The Reynolds number, Re, is constant for all models. Having a constant Re provides a consistent flow field for all models, in which all simulations receive the same amount of mixing, and grounds for fair comparison between simulations. The nondimensional reaction rate, $R^{*}$, is the metric by which all models are compared. The remaining four parameters are the Damköhler number, Da, the separation distance between scalars, $s^{*}$, the scalar filament width, $f^{*}$, and the Schmidt number, Sc. These are the parameters 
that are each varied, in turn, in a series of simulations to determine the effect on the reaction rate. The parameters $s^{*}$ and $f^{*}$ change the scalar release geometry, Sc effectively changes the diffusion, and Da effectively changes the reaction constant. 


\section{Chapter 3}

\section{Numerical Model and Postprocessing}

\subsection{Numerical Model}

The two-dimensional numerical model is created in the finite element analysis software COMSOL Multiphysics which approximates the solution to flow and transport phenomena. The model uses the Navier-Stokes equations to solve the flow field while simultaneously solving a pair of coupled advection-diffusion-reaction equations for the transport and reaction of two scalar concentrations. In this study, two models are created: a base model without a cylinder, and a model with a cylinder and associated wake structure. The geometry and parameters of both models are the same, with the exception of the absence of the cylinder in one model and presence of the cylinder in the other model.

\subsubsection{Cylinder Model}

The model domain is a rectangle with height $0.22 \mathrm{~m}$ and length $0.37 \mathrm{~m}$ shown in Fig. 3.1. The cylinder, of diameter $\phi=0.0127 \mathrm{~m}$, is centered at the origin of the coordinate system. The height of the domain puts the lateral boundaries a distance of 8 cylinder diameters away from the cylinder. This distance is sufficient to minimize the effects of these boundaries on the cylinder wake[65]. 


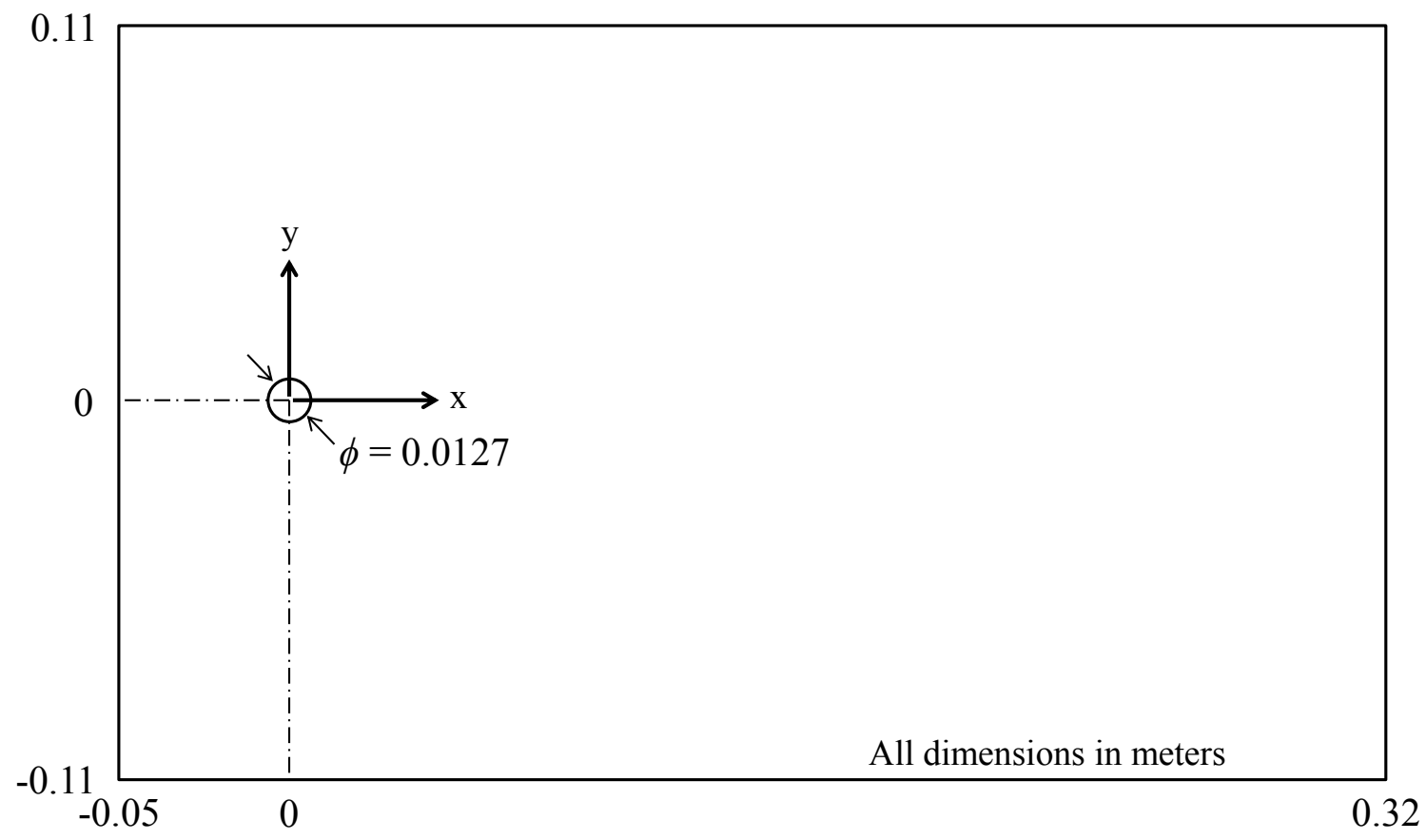

Figure 3.1: Geometry and dimensions of the model where the origin of the coordinate system is at the cylinder center.

Using COMSOL's free mesher with governing parameters listed in Appx. A, an inhomogeneous unstructured mesh of triangular elements is fitted to the domain. The flow field, having smooth gradients, uses a coarse mesh that is made up of less than 10,000 elements. The scalar field is more complicated, requiring the mesh be refined to approximately 85,000 elements. The mesh need only be refined in the cylinder wake region. Therefore, the domain is divided into four subdomains, shown in Fig. 3.2, where, regions 1 and 3 have a very refined mesh (maximum element length of $0.0012 \mathrm{~m}$ ), and regions 2 and 4 have a coarser mesh (maximum element length of $0.009 \mathrm{~m}$ ). The smallest mesh elements are around the cylinder where there is a no slip condition, while the largest elements are near the lateral boundaries. This variable mesh grid optimizes having a refined mesh to improve model accuracy while minimizing the total number of mesh elements to reduce computation time. 


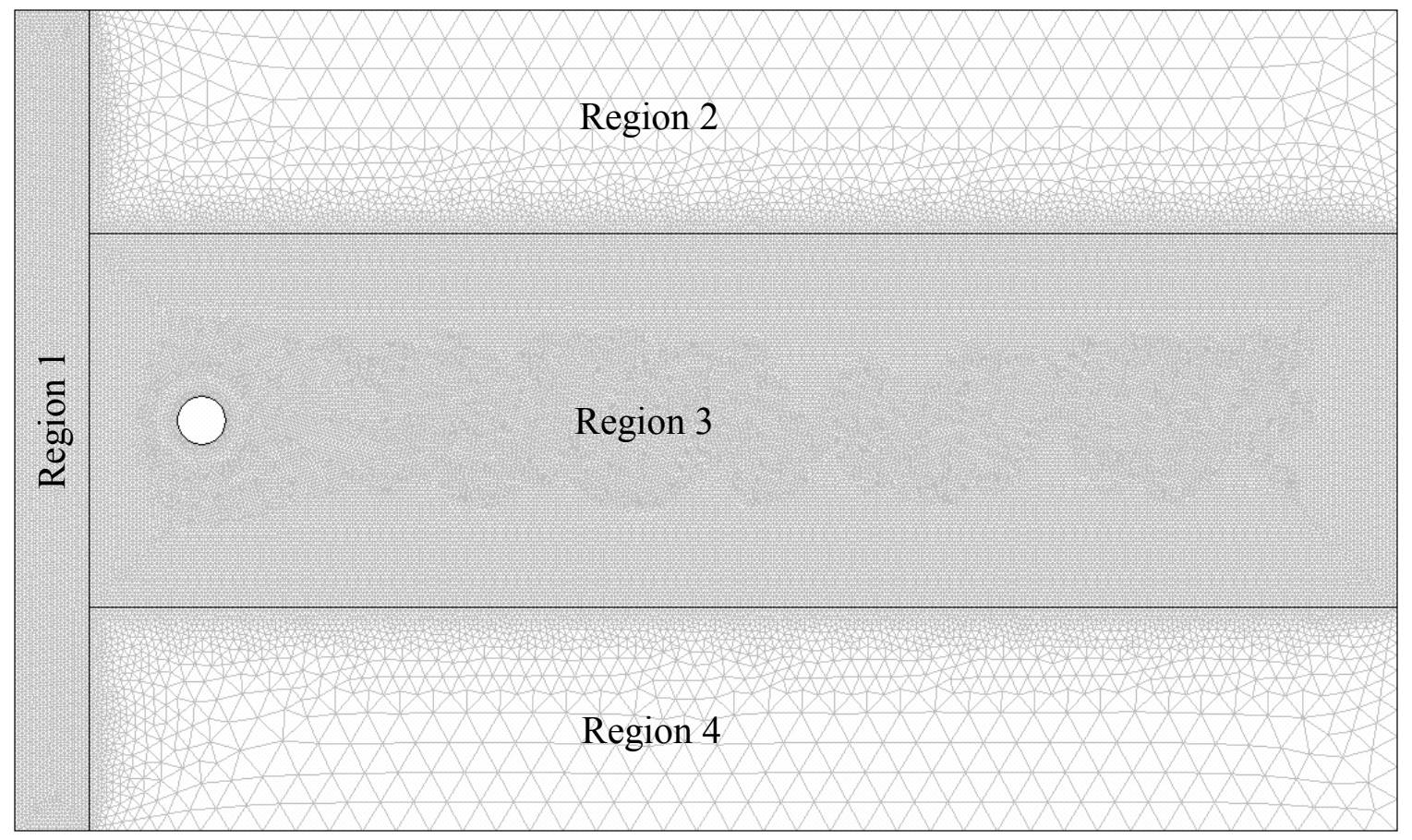

Figure 3.2: Model mesh grid generated using Comsol free mesher. Regions 1 and 3 have very refined mesh and regions 2 and 4 have a coarse mesh.

\subsubsection{Flow Field}

The boundary conditions in the model are set such that flow enters the domain at the left boundary $(x=-0.05 \mathrm{~m})$, flows to the right, and then exits the domain at the right boundary $(x=0.32 \mathrm{~m})$. The inflow boundary condition is defined by the normal velocity equation $\mathbf{u}=-u_{0} \mathbf{n}$, where, $\mathbf{u}$ is the velocity field and $\mathbf{n}$ is normal to the boundary. The outflow boundary condition specifies that viscous stresses vanish according to $\eta\left(\nabla \mathbf{u}+(\nabla \mathbf{u})^{T}\right) \mathbf{n}=\mathbf{0}$, with a Dirichlet condition on the pressure $p=p_{0}$, where $\eta$ is dynamic viscosity, and $p_{0}$ is pressure. The upper and lower lateral boundaries are walls specified with a free slip condition to give the simulation the effect of being run in an infinite domain. The free slip condition on the walls can be defined as $\mathbf{u} \cdot \mathbf{n}=0$ and $\mathbf{t} \cdot\left(-p \mathbf{I}+\eta\left(\nabla \mathbf{u}+(\nabla \mathbf{u})^{T}\right)\right) \mathbf{n}=0$, where, $\mathbf{t}$ is a tangential vector to the boundary. The cylinder is given a no slip boundary condition defined as $\mathbf{u}=\mathbf{0}$.

The initial flow condition is $\mathbf{u}(x, y, t)=(0,0,0)$. As the simulation begins, and time starts 
moving forward, the velocity is ramped up from zero to the maximum velocity over a period of 0.2 seconds, using a smoothed Heaviside function with a continuous first derivative.

The Navier-Stokes equations are used to solve the flow field around the cylinder and are given as

$$
\begin{gathered}
\frac{\partial \mathbf{u}}{\partial t}+\mathbf{u} \cdot \nabla \mathbf{u}=-\frac{1}{\rho} \nabla p+\nu \frac{\partial^{2} \mathbf{u}}{\partial x^{2}}+g \\
\nabla \cdot \mathbf{u}=0
\end{gathered}
$$

where, $\mathbf{u}$ is the velocity field, $\rho$ is density, $\nu$ is kinematic viscosity, and $g$ is gravity. Equation 3.1 is the momentum transport equation and Eqn. 3.2 is the equation of continuity for incompressible fluids. The Reynolds number for the model is $\mathrm{Re}=97.4$, which generates a laminar flow with well developed vortex shedding and facilitates scalar field calculations. Furthermore, the model is used in comparisons with experimental work done at this Re.

In this time-dependent model, a transient analysis is used to solve the developing unsteady flow field from $t=0$. The Direct (PARDISO) linear system solver uses Gaussian elimination to solve the systems of linear equations that are generated in each time step. The Generalized- $\alpha$ timestepping method is used with a time step based on the Courant-Friedrichs-Lewy (CFL) number. The solver uses the CFL number in the form $u \Delta t / \Delta x=1$, where, $\Delta x$ is the length of the mesh element. Using this relationship, the time step is based on the advection time across an element. The dependence on the element size creates a spatial-temporal relationship: directly controlling the element size in the mesh grid, indirectly changes the solver time step such that as the mesh size decreases, the time step decreases.

Streamlines from the resulting flow field $(u, v)$, are shown in Fig. 3.3a. To reveal the underlying structure of the vortex wake, most of the mean velocity in the streamwise direction is removed, $\left(u-0.95 u_{0}, v\right)$, with the resulting streamlines shown in Fig. 3.3b. 
(a)

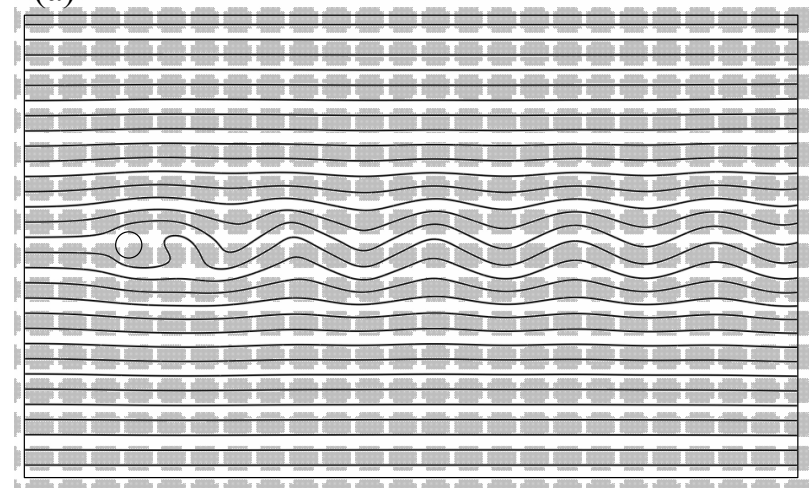

(b)

Figure 3.3: (a) Velocity streamlines for the flow field, and (b) velocity streamlines shown with most the mean velocity in the streamwise direction removed revealing the vortex wake structure.

The flow model is simulated for multiple 30-second runs. Each consecutive run uses the flow field results of the previous run as the starting point for the next run. This gives the end effect of having run the simulation for a few minutes. To verify convergence to a quasi-steady-state, we examine the velocity. A 30-second time history of the velocity at an arbitrary point in the cylinder wake is plotted in Fig. 3.4. On the plot, three consecutive 30-second time histories are overlaid. The three curves align with no visible discrepancies showing that the period is not changing, and the flow field has reached a quasi-steady-state. 


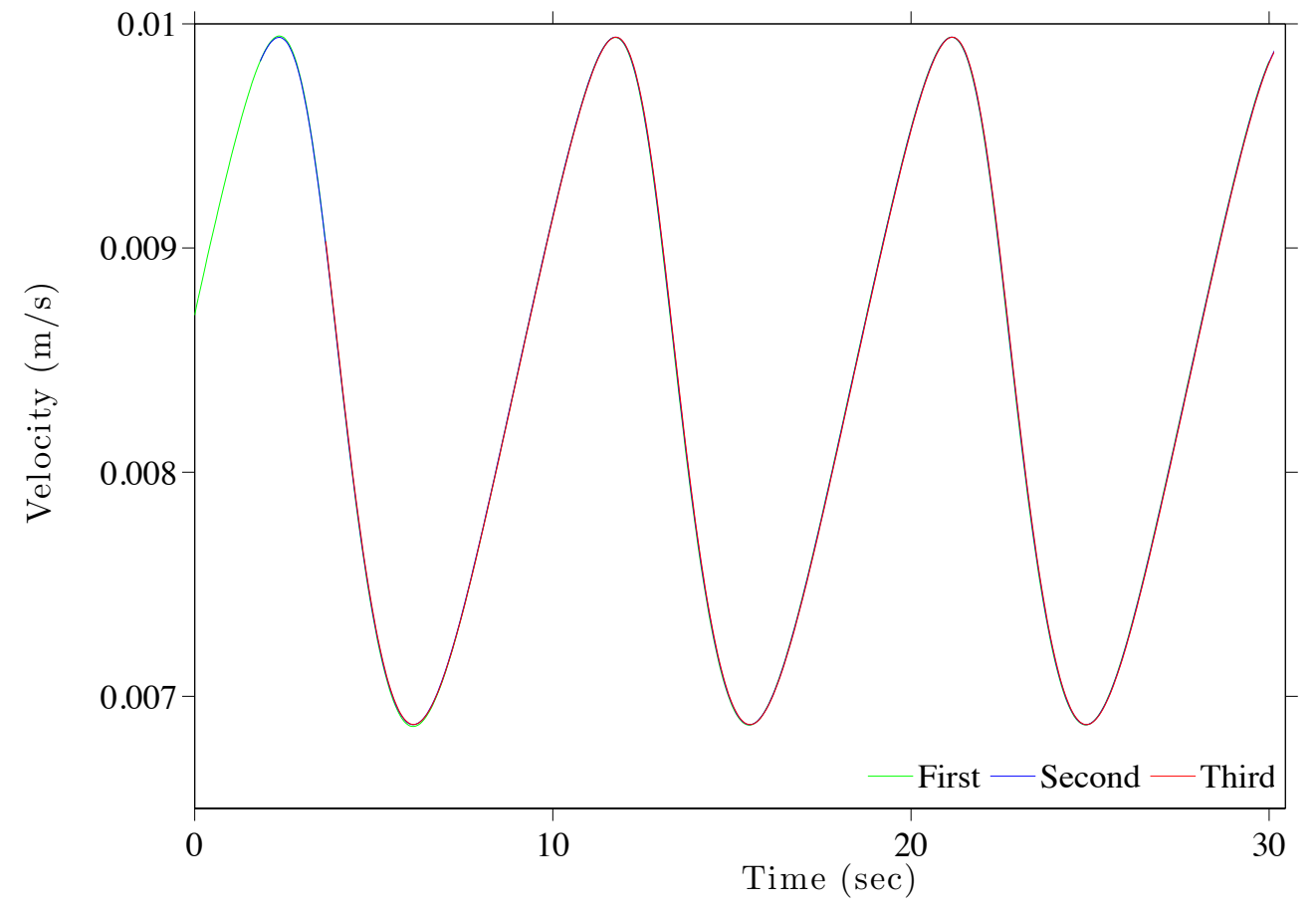

Figure 3.4: Time history of the velocity at a point in the cylinder wake shown for three consecutive 30-second simulations overlaid to show convergence.

With the model running at quasi-steady-state, the shedding period is determined using a velocity plot similar to Fig. 3.4. The shedding period is the time it takes for a pair of vortices to fully form and detach from the cylinder. On the velocity plot, the period is the time to complete one oscillation cycle which is determined to be 9.24 seconds. Using Eqn. 1.1, the nondimensional shedding frequency is calculated as $\mathrm{St}=0.18$. For the well known cylinder flow at $\mathrm{Re}=100$, it is expected that St be closer to $0.16[42,66,67]$. The mild discrepancy may be due to the location of the lateral boundaries which, if placed too close to the cylinder, can cause St to become artificially high[65].

\subsubsection{Scalar Transport}

The scalar concentrations are introduced continuously into the flow upstream of the cylinder at the actual release location of $x=-0.03 \mathrm{~m}$. The $C_{1}$ and $C_{2}$ scalars have a lateral separation 
distance, $s$, which is symmetric about the stagnation streamline. Each concentration has a filament width, $f$. These dimensions are shown in Fig. 3.5

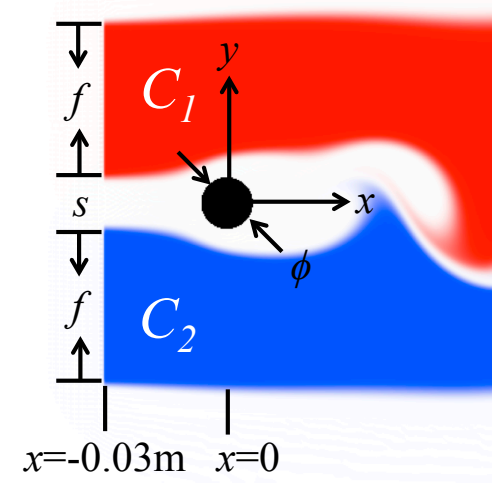

Figure 3.5: Scalar release geometry and dimensions for two scalar concentrations, $C_{1}$ and $C_{2}$, released into the flow with scalar separation, $s$, and filament width, $f$.

The inlet boundary condition is scalar concentration defined as $C=C_{0}$, where, $C_{0}$ is the maximum concentration specified as a model variable. The boundary condition on the outlet is convective flux defined as $\mathbf{n} \cdot(-D \nabla C)=0$. Under this condition, scalars are transported out of the domain by convection only. The lateral boundaries and the boundaries on the cylinder all have the condition of insulation/symmetry, or no concentration flux, defined as $\mathbf{n} \cdot(-D \nabla C+C \mathbf{u})=0$. The internal domain boundary conditions all have the continuity condition defined as $\mathbf{n} \cdot\left(\mathbf{N}_{1}-\mathbf{N}_{2}\right)=0$, where, $\mathbf{N}$ is the scalar flux and is continuous in the normal direction across the boundary.

The initial condition for the scalar concentration is $C_{1}(x, y, t)=C_{2}(x, y, t)=0$. When the scalars are introduced to the flow, the concentration distribution has a "top hat" profile with sharp corners, and must be smoothed before they can be solved in the simulation. We applied diffusion to the scalar concentrations before they are released, effectively creating a virtual release location farther upstream at $x=-0.04795 \mathrm{~m}$, shown in Fig. 3.6. The net effect is that the scalars behave as if they are released at the virtual release location with the "top hat" profile shown in Fig. 3.7a, and advect and diffuse, having a slightly smoother gradient shown in Fig. 3.7b. A set of error function 
equations are developed to define the inlet release of the scalar concentrations, and are given as

$$
\begin{gathered}
C_{1}=\frac{C_{1}^{0}}{2}\left[\operatorname{erf}\left(\frac{y-s / 2}{\sqrt{4 D x / u_{0}}}\right)-\operatorname{erf}\left(\frac{y-f-s / 2}{\sqrt{4 D x / u_{0}}}\right)\right] \\
C_{2}=\frac{C_{2}^{0}}{2}\left[\operatorname{erf}\left(\frac{y+f+s / 2}{\sqrt{4 D x / u_{0}}}\right)-\operatorname{erf}\left(\frac{y+s / 2}{\sqrt{4 D x / u_{0}}}\right)\right] .
\end{gathered}
$$

Using these equations, the scalar concentration profiles are shown at the virtual and actual release locations in Fig. 3.7.

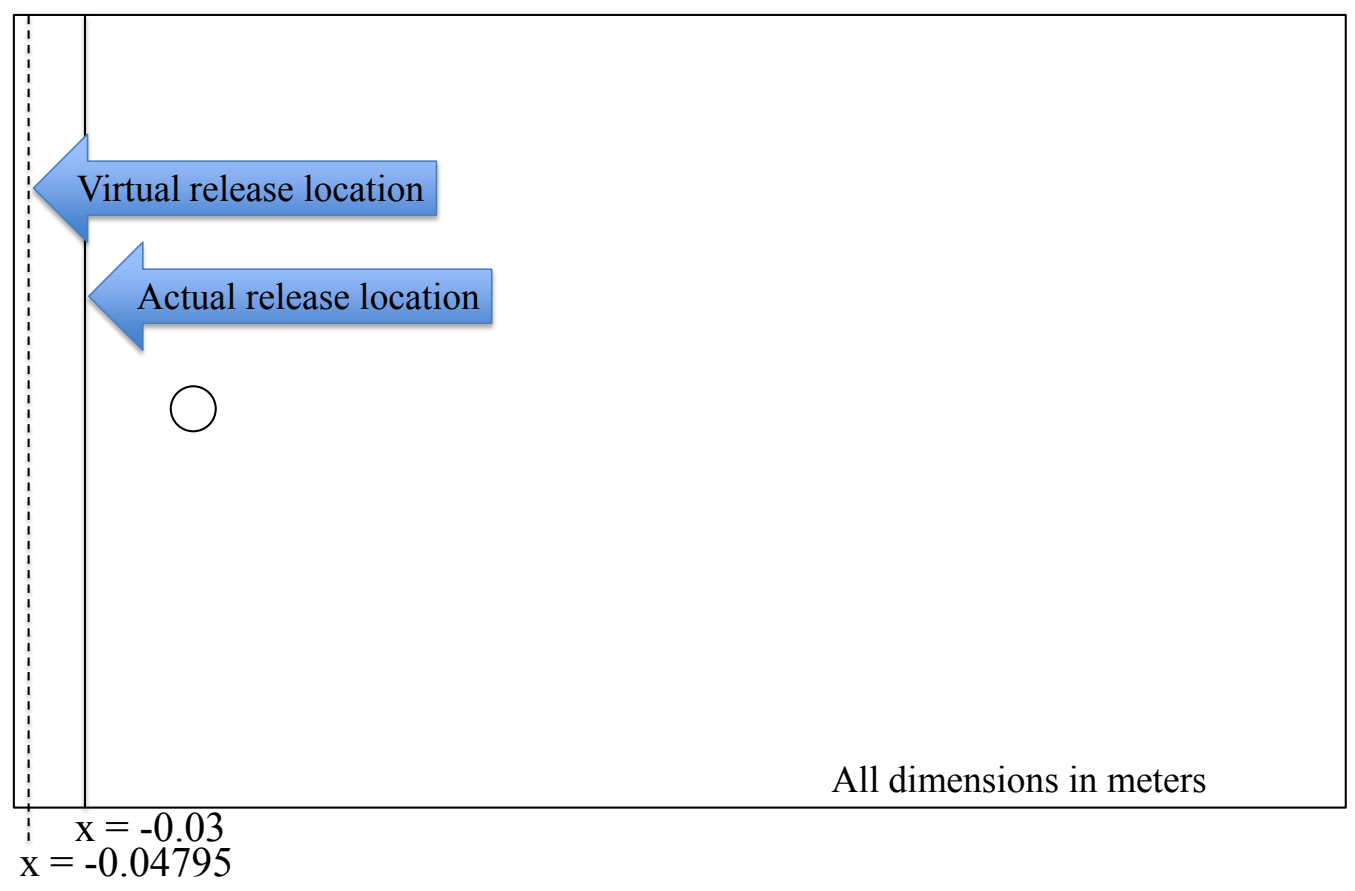

Figure 3.6: Virtual and actual scalar release locations in model domain. 


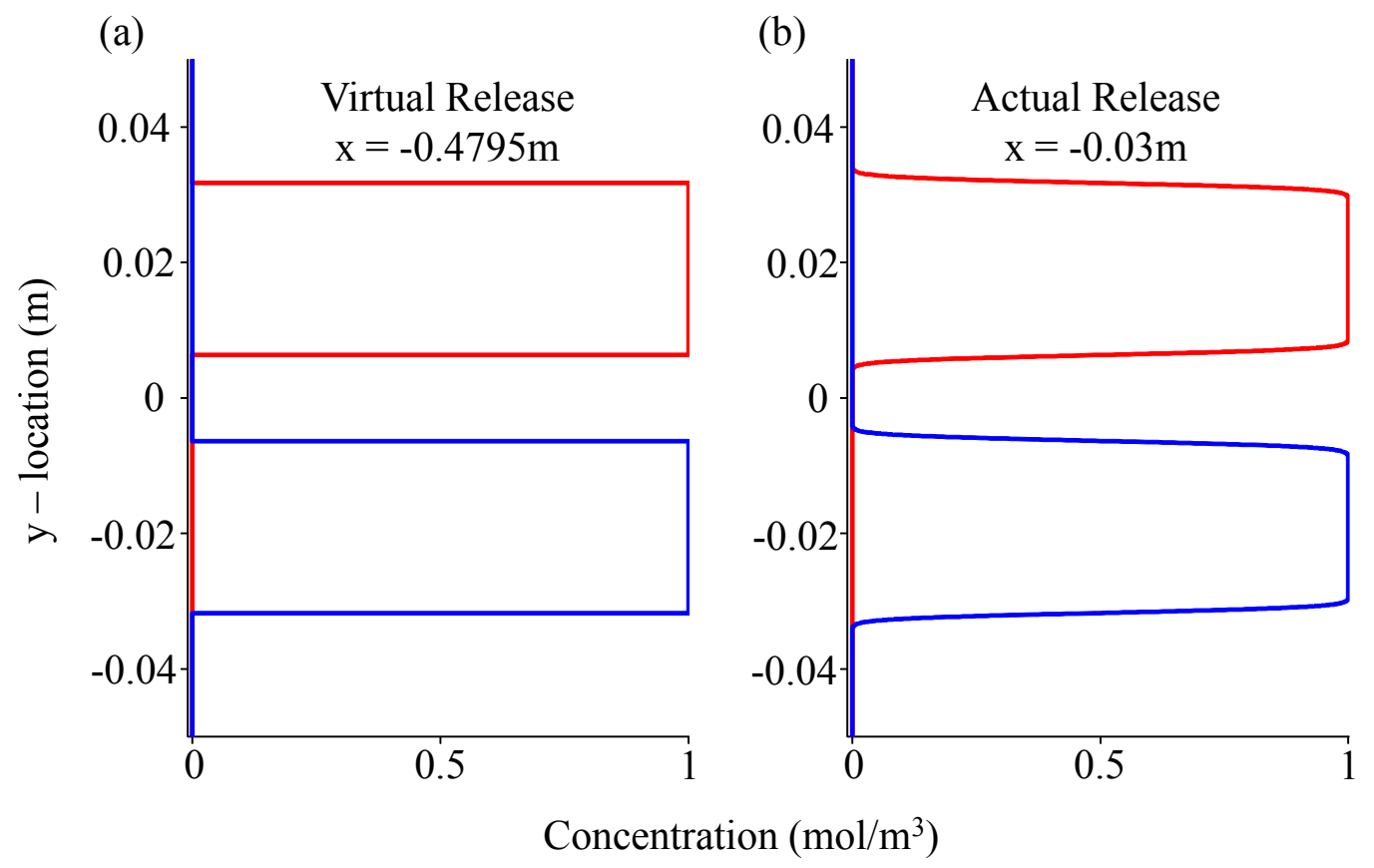

Figure 3.7: Concentration profiles at virtual and actual scalar release locations.

The equation governing the scalar transport of $C_{1}$ and $C_{2}$ is the advection-diffusion-reaction equation defined for both scalars as

$$
\begin{aligned}
& \frac{\partial C_{1}}{\partial t}+\mathbf{u} \cdot \nabla C_{1}-D \nabla^{2} C_{1}=R \\
& \frac{\partial C_{2}}{\partial t}+\mathbf{u} \cdot \nabla C_{2}-D \nabla^{2} C_{2}=R
\end{aligned}
$$

where, $C_{1}$ and $C_{2}$ are the respective scalar concentrations, $\mathbf{u}$ is the velocity field, $D$ is molecular diffusivity, and $R$ is the reaction between the scalars, which couple these two equation together. We specify a second order reaction rate

$$
R=k C_{1} C_{2}
$$

where, $k$ is a reaction rate constant.

Once the scalars are released into the flow, they advect with the developed flow field past the cylinder and into the vortex street, moving downstream as a wave front. The advective time scale is the time it takes for the scalars to advect across the domain and exit at the domain outlet. In 
this model, the advective time scale is about 50 seconds. It takes an additional 10 seconds for the transient scalars to pass out of the domain and reach a quasi-steady-state. The scalars develop into a unsteady, yet stable and period flow, thus called quasi-steady-state. Finally, we allow one more shedding cycle to pass out of the domain. During the last 10 seconds, the flow field and scalar field are at quasi-steady-state. This is the time range from which all data is processed. Figure 3.8 is an instantaneous image of the simulation showing (a) the $C_{1}$ and $C_{2}$ scalar concentrations, and (b) the reaction rate between the scalars, where (a) and (b) are shown at the same time phase.

(a)

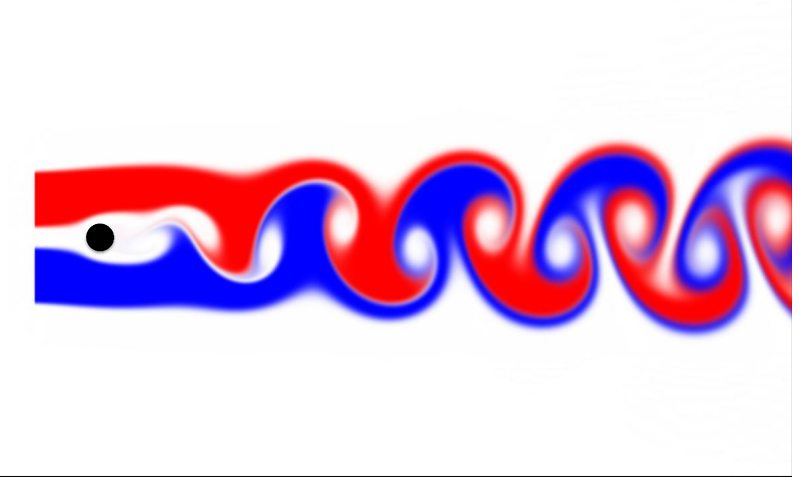

(b)

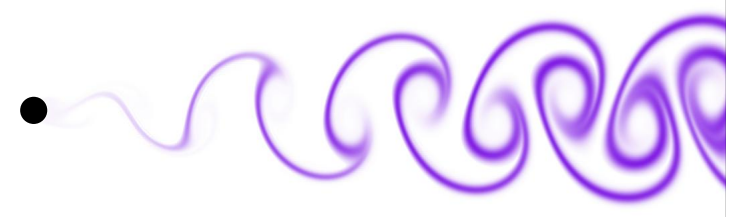

Figure 3.8: (a)Instantaneous $C_{1}$ and $C_{2}$ scalars at quasi-steady-state. Scalars highlight the welldeveloped vortex street, and (b)associated reaction rate.

\subsubsection{Sensitivity Analysis}

We explored the effect of changing the total number of mesh elements by refining the mesh in regions 1 and 3 . The models tested have a total number of elements ranging between approximatley 10,000 and 150,000. The horizontal centerline reaction profiles for four models are plotted in Fig. 3.9, showing that simulation accuracy improves with an increase in total number of elements. The profile for each model is integrated and plotted against the inverse of the number of elements squared in Fig. 3.10. As the total number of elements in the model increases to very large numbers, the integrated reaction converges, suggesting that past a certain large number of elements, the model will become insensitive to mesh refinement. 


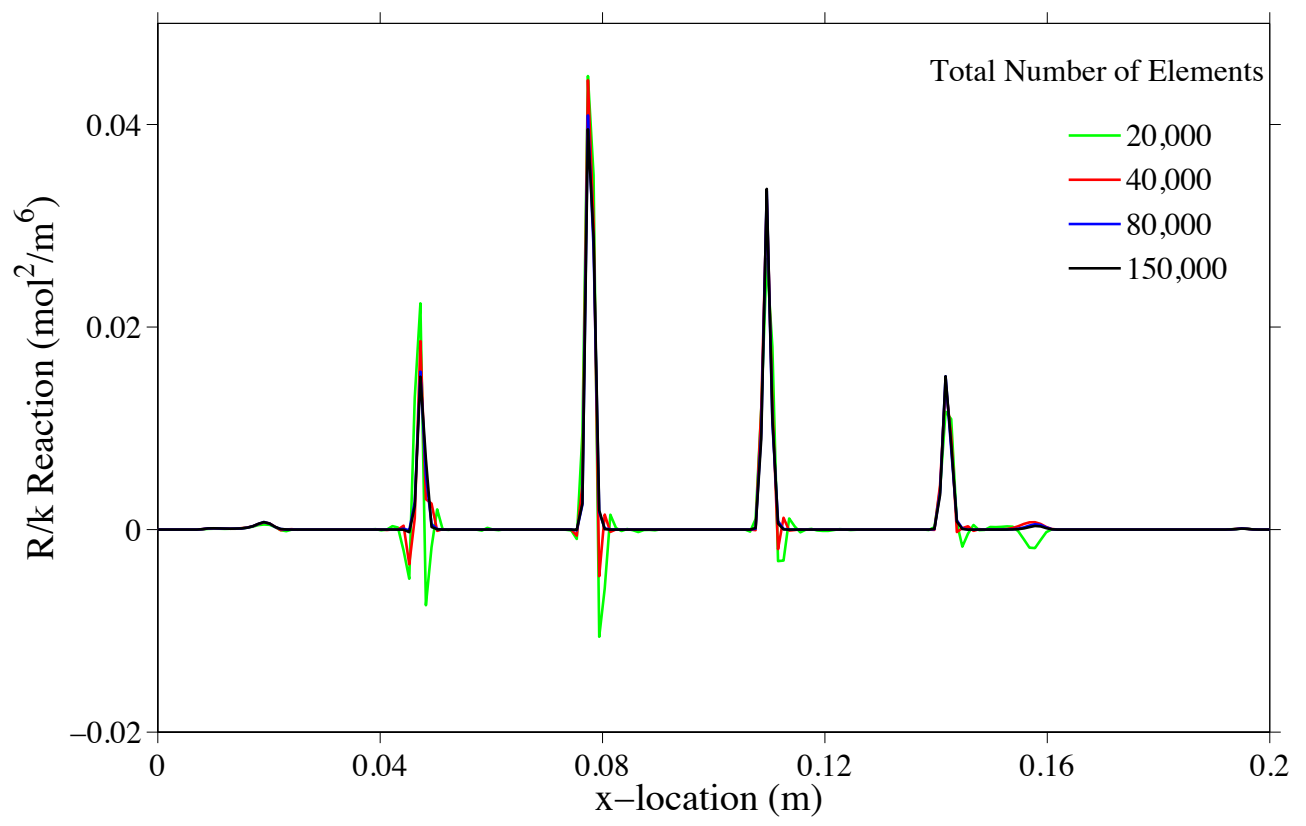

Figure 3.9: Reaction concentration profiles along the centerline for models with varying number of total mesh elements.

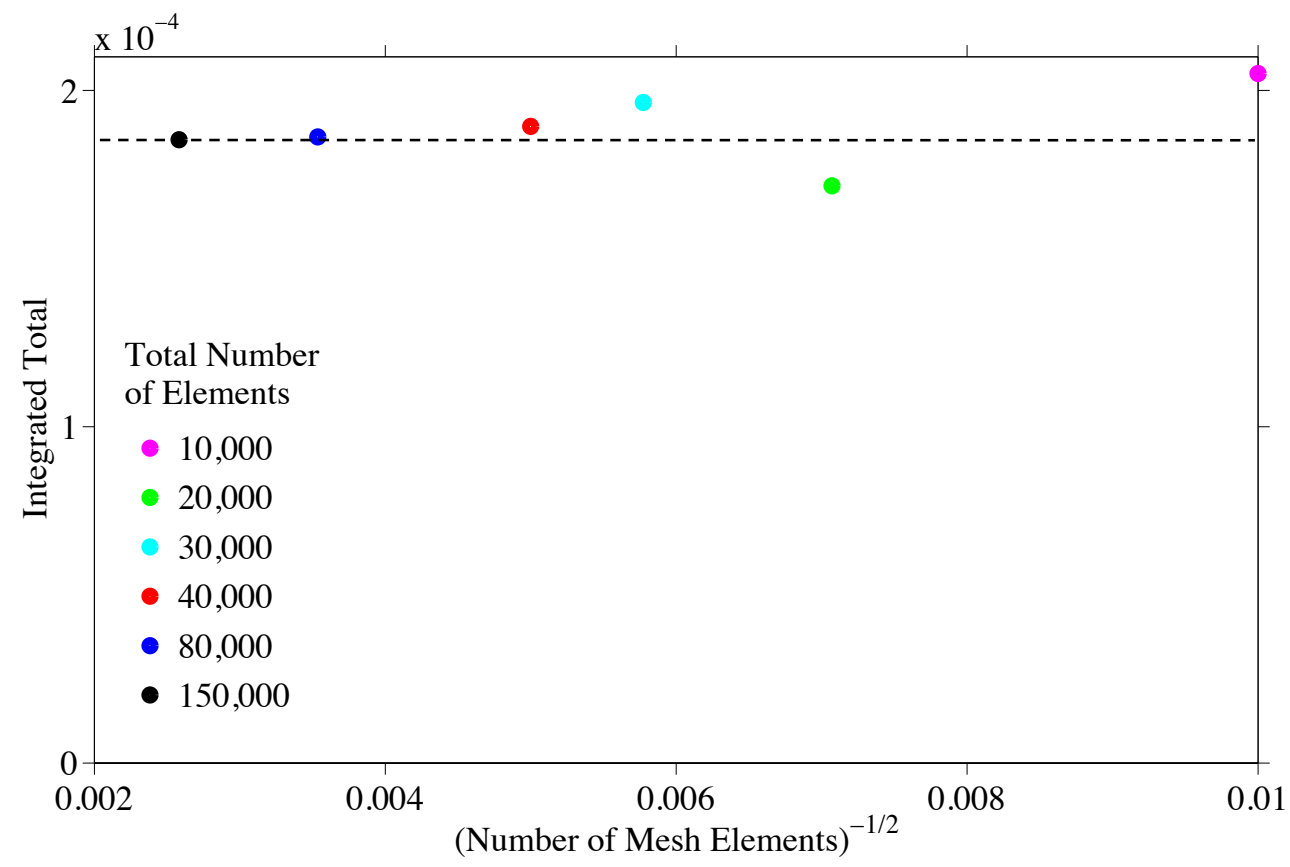

Figure 3.10: Reaction concentration profiles along the centerline for models with varying number of total mesh elements. 


\subsubsection{No-Cylinder Model}

Using the cylinder model, a "no-cylinder" model was created as a base-line case for comparison with the cylinder case. This model is identical to the cylinder model with the exception of the absence of the cylinder. All geometries and parameters are the same in both models. The boundary conditions, initial conditions, and the mesh are the same in both models. With no cylinder to disrupt the flow, the flow field reaches steady-state with a uniform inflow velocity profile producing parallel streamlines across the entire domain. The transport model in Fig 3.11a shows the scalar field at steady-state where simple one-dimensional advection carries the scalars downstream. Molecular diffusion is the only mixing mechanism, as the flow field exhibits no stirring. The initial separation between the scalars is relatively small, however, since there is only diffusion, the resulting reaction is also small, shown in Fig. 3.11b as a thin filament.

(a)

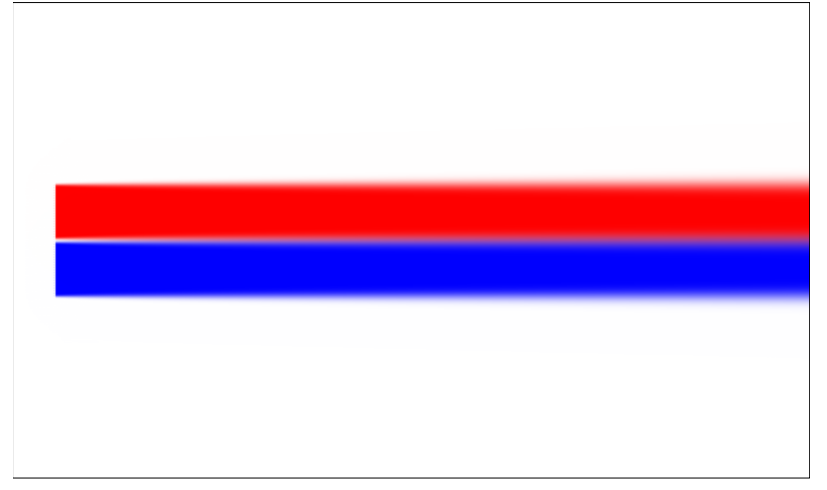

(b)

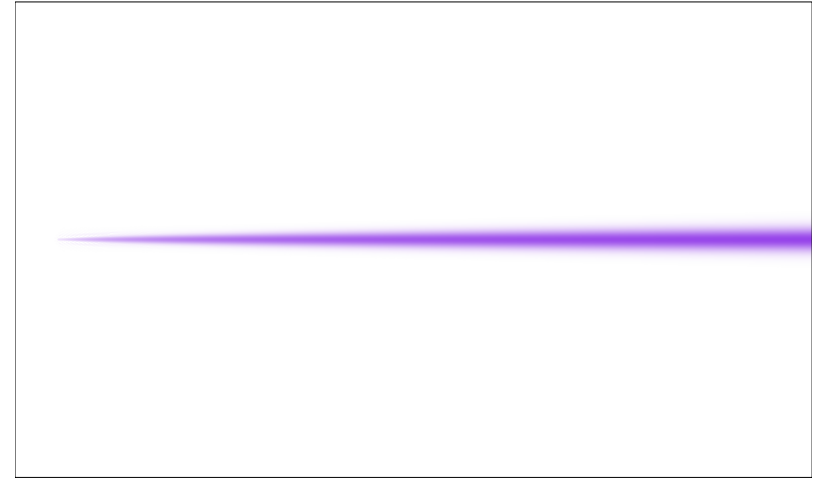

Figure 3.11: (a) Scalar concentrations $C_{1}$ and $C_{2}$ and (b) $R / k$ reaction images for the no-cylinder model at steady-state.

This simple flow field allows for the analytical transport and reaction equations to be solved. Figure 3.12 shows the scalar field and the locations at which three cross-sections are taken: one near the release $(x=-0.029 \mathrm{~m})$, one mid-stream $(x=0.1 \mathrm{~m})$, and one down-stream $(x=0.31 \mathrm{~m})$. The cross-sectional profiles of the scalar concentrations using the analytical solution are shown in Fig. 3.13 as the solid lines. At corresponding locations, concentration profiles determined using the numerical simulation solution are plotted in Fig. 3.13 as the broken lines. The match between the 
analytical solution and the model solution shows that the simulation is accurate, and validates the model for the cylinder case.

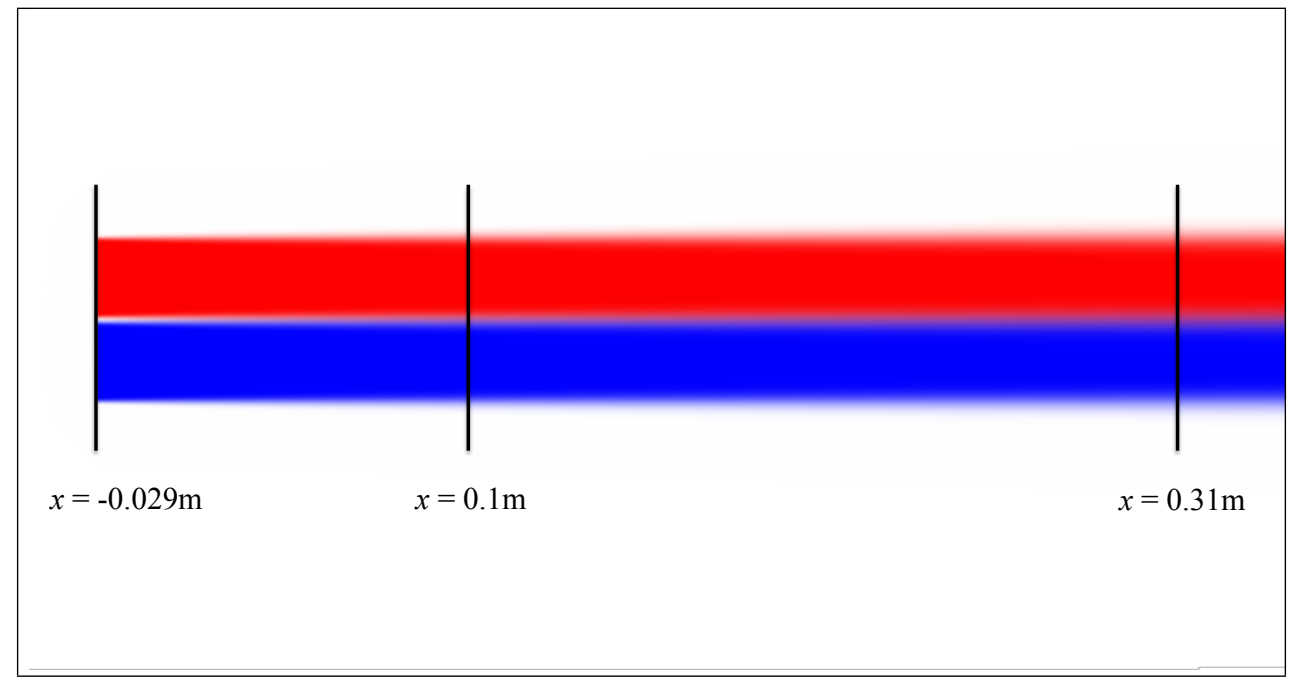

Figure 3.12: $C_{1}$ and $C_{2}$ scalar transport model at steady-state with three locations marked. Concentration profiles at these location are shown in Fig. 3.13 

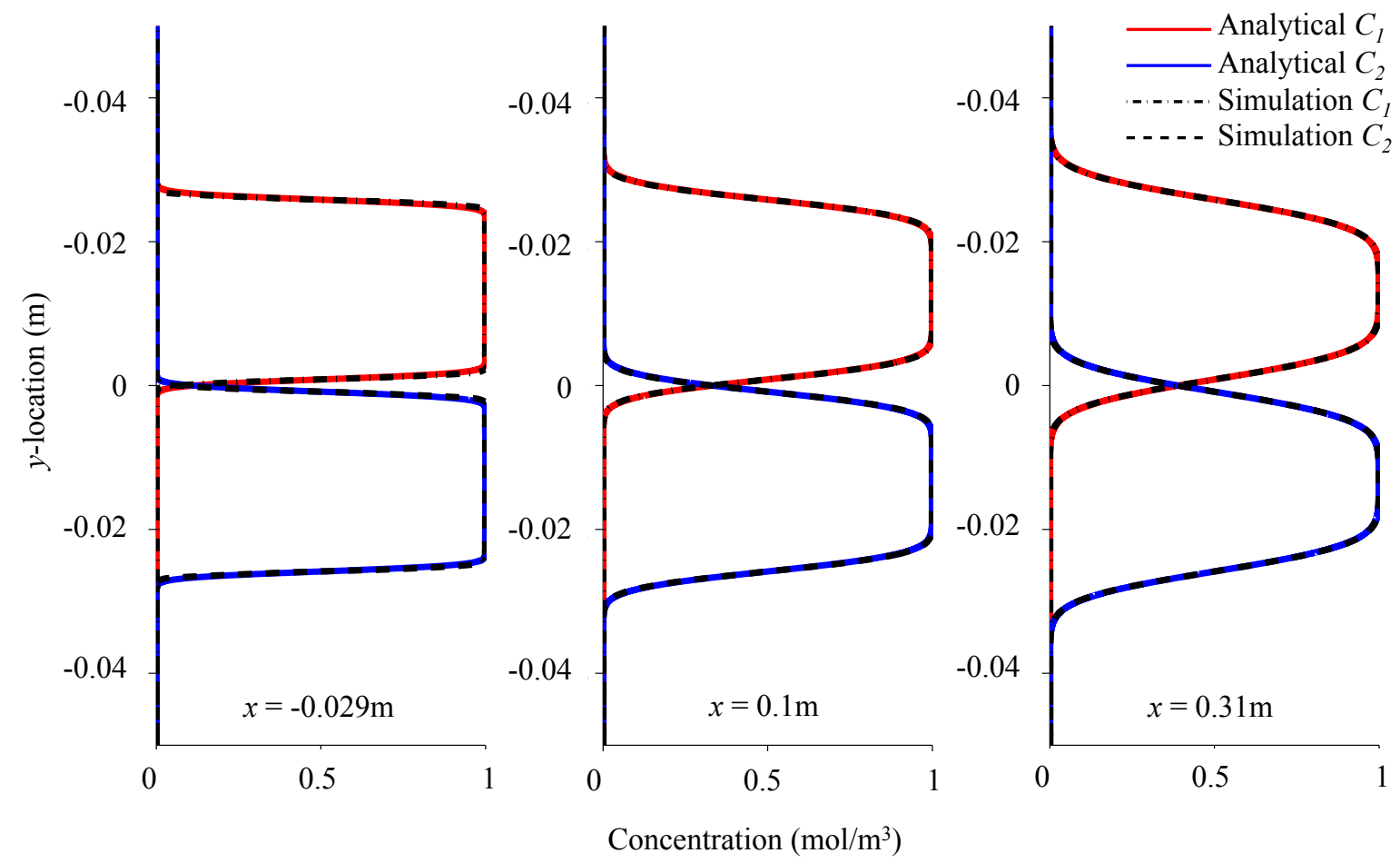

Figure 3.13: $C_{1}$ and $C_{2}$ scalar concentration profiles of Comsol data and analytical solution for three $x$-locations. The agreement between the analytical solution and the numerical simulation validates the model.

\section{2 $\quad$ Postprocessing Analyisis}

The reaction data for one shedding period is scaled by the reaction constant, $k$, giving just the $C_{1} C_{2}$ reaction product. This data is divided into 50 time phases and exported from COMSOL to be processed in MATLAB. At each time phase, the data is integrated laterally to remove the effect of the infinite domain. The data are then averaged over time resulting in the total reaction in one shedding period across the domain. This total reaction quantity, plotted with streamwise location, is the metric by which all the models are compared. The MATLAB codes are in Appx. B. 


\section{Chapter 4}

\section{Reactions Without Cylinder Wake}

The simple no-cylinder model is used to compare model reaction results with the analytical solution as well as the numerically integrated solution, and validate the numerical simulations. The model also supports the scaling relationships that are identified for the reaction between scalars. Additionally, reaction rates from this simple model are used as the baseline case in comparisons with the reaction rates in the cylinder wake simulations.

\subsection{Limiting Reaction Rate Maximum}

The simple model without the cylinder has a rectilinear flow field allowing the exact analytical solution to be solved as the limiting case of large $f$ and small Da, where $f$ is the scalar filament width at the release, and $\mathrm{Da}$ is the Damköhler number. In the limiting case, $f$ is large to ensure there are sufficient scalars available and that reaction is not limited by scalar depletion. Moreover, since reaction will only take place where both scalars exist, the "large $f$ " constraint only must satisfy $f_{s}^{*} \gg \lambda$, which requires that the scalars diffuse a distance greater than their separated distance. The second constraint in this limiting case, Da $\ll 1$, decouples the paired ADR equations allowing them to be solved individually. Applying these constraints to Eqns. 2.17 and 2.18, the concentration equations simplify to

$$
\begin{aligned}
& C_{1}^{*}=\frac{1}{2}\left[1+\operatorname{erf}\left(\frac{y_{s}^{*}-1 / 2}{\lambda / 2}\right)\right] \\
& C_{2}^{*}=\frac{1}{2}\left[1+\operatorname{erf}\left(\frac{-y_{s}^{*}-1 / 2}{\lambda / 2}\right)\right]
\end{aligned}
$$


where, recall from Chapter $2, y_{s}^{*}=y / s$ and $\lambda=\sqrt{x_{s}^{*} / \text { Pe }}$. Using these expressions for concentration, the equation for the integrated nondimensional reaction for the steady-state model, Eqn. 2.22, can be written out as

$$
\mathcal{R}^{*}=\frac{1}{4} \int_{-\infty}^{\infty}\left[1+\operatorname{erf}\left(\frac{y_{s}^{*}-1 / 2}{\lambda / 2}\right)\right]\left[1+\operatorname{erf}\left(\frac{-y_{s}^{*}-1 / 2}{\lambda / 2}\right)\right] \mathrm{d} y_{s}^{*} .
$$

The integral can be rewritten by letting $\xi=2 y_{s}^{*} / \lambda$ and $\alpha=-1 / \lambda$,

$$
\mathcal{R}^{*}=\frac{\lambda}{8} \int_{-\infty}^{\infty}[1+\operatorname{erf}(\xi+\alpha)][1+\operatorname{erf}(-\xi+\alpha)] \mathrm{d} \xi
$$

In this form, the integral has the exact solution (solution is in Appx. C)

$$
\int_{-\infty}^{\infty}[1+\operatorname{erf}(\xi+\alpha)][1+\operatorname{erf}(-\xi+\alpha)] \mathrm{d} \xi=4\left[\frac{1}{\sqrt{2 \pi}} \exp \left(-2 \alpha^{2}\right)+\alpha \operatorname{erf}(\sqrt{2} \alpha)+\alpha\right]
$$

Using the integral identity, Eqn. 4.4 can be written

$$
\mathcal{R}^{*}=\frac{\lambda}{2}\left[\frac{1}{\sqrt{2 \pi}} \exp \left(\frac{-2}{\lambda^{2}}\right)-\frac{1}{\lambda} \operatorname{erf}\left(\frac{-\sqrt{2}}{\lambda}\right)-\frac{1}{\lambda}\right] .
$$

Equation 4.6 is the exact analytical solution for the reaction rate as a function of the single parameter, $\lambda$ (subject to $f_{s}^{*} \gg \lambda$ and $\mathrm{Da} \ll 1$ ). This analytical solution is plotted in Fig. 4.1, on (a) linear axes and (b) log-log axes.

(a)

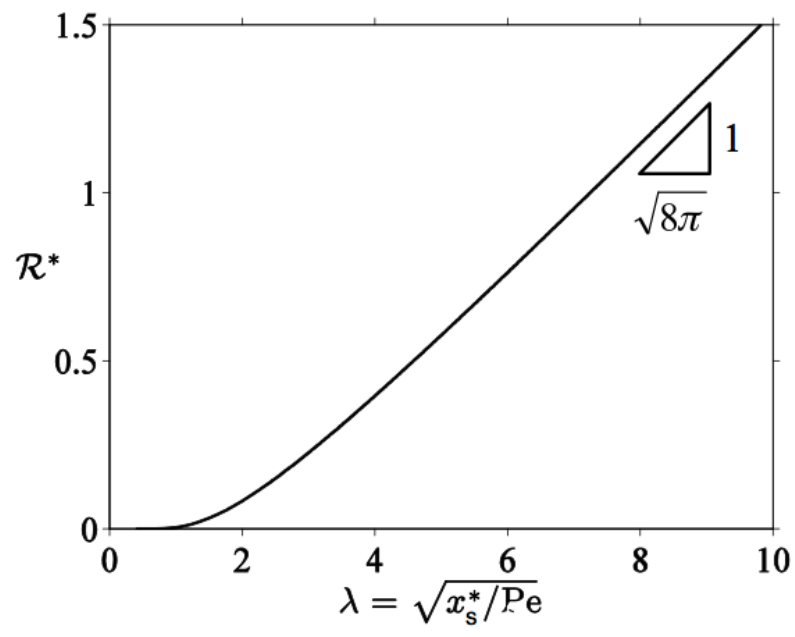

(b)

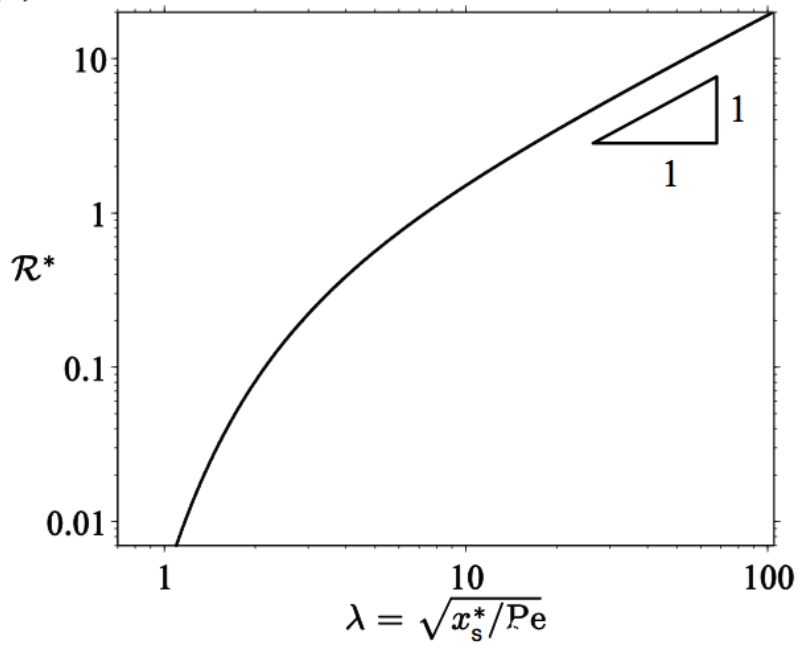

Figure 4.1: Analytical solution for reaction rate as a function of $\lambda$ on (a) linear scale, and (b) log-log scale, showing the limiting case for the maximum reaction rate. 
Recall that $\lambda$ is the ratio of the laterally diffused distance at a downstream location to the separation distance between the scalars. For $\lambda<1$, the scalars have not yet diffused the length of the gap between them and there is no reaction. At $\lambda \approx 1$, the scalars have just reached the centerline between them and begin to react, shown in Fig. 4.1a as the reaction rate diverges from zero. For $\lambda \gg 1$, the scalars have diffused well across the centerline and react at a linearly increasing rate with $\lambda$. Solving Eqn. 4.6 for $\lambda \rightarrow \infty$, gives the limiting reaction rate

$$
\mathcal{R}^{*}=\frac{\lambda}{\sqrt{8 \pi}}=\frac{1}{\sqrt{8 \pi}} \sqrt{\frac{x_{s}^{*}}{\mathrm{Pe}}} .
$$

Figure $4.1 \mathrm{~b}$ shows the same relationship plotted on log-log axes revealing a slope of unity. The use of power laws further reveal that reaction rate scales linearly with $\lambda$. Using the definition of $\lambda$, the reaction rate, more specifically, scales as $x_{s}^{* 1 / 2}$ for the limiting case of $f_{s}^{*} \gg \lambda$ and Da $\ll 1$.

\subsection{Effect of $f$ and Da}

The constraints on the analytical solution are relaxed to investigate the effects of $f_{s}^{*}$ and Da on the reaction rate. The first constraint on the analytical solution is $f_{s}^{*} \gg \lambda$. The effect of varying $f_{s}^{*}$ away from this constraint, (while keeping Da $\ll 1$ ), is determined using the numerically integrated solution and the numerical simulation. Using Eqn. 2.22, the reaction rate equation for the steady-state problem, with the concentration equations 2.17 and 2.18, the integrated reaction rate is calculated for a series of $f$ values, where $f_{s}^{*}=14.7,2.94,1.47$, and plotted as dashed lines in Fig. 4.2. 


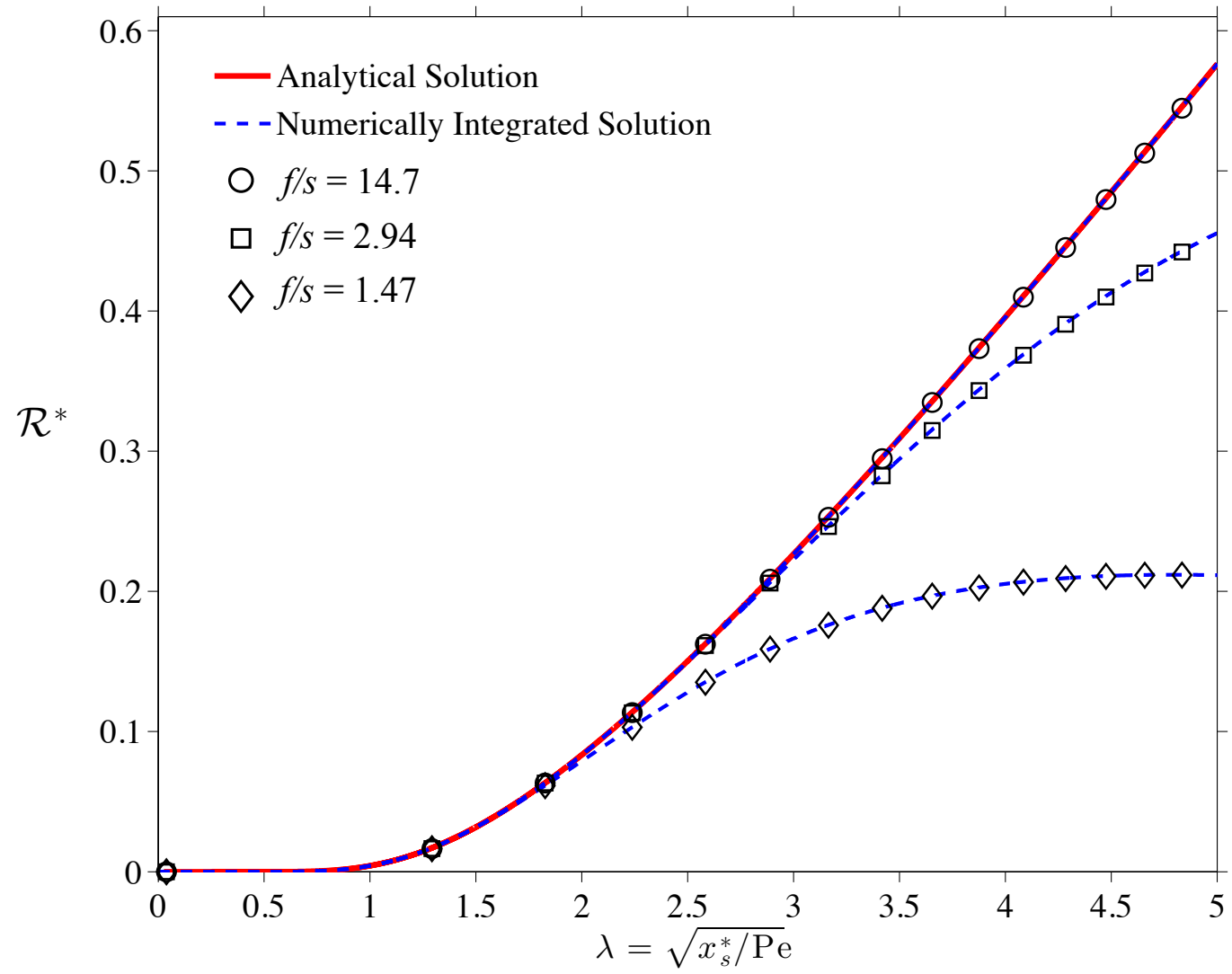

Figure 4.2: Integrated reaction rate for the no-cylinder case for $f^{*}=14.7,2.94$, and 1.47 , where, the analytical solution is the solid line, the dashed lines are the numerically integrated solution, and the symbols are the simulation results.

A series of numerical simulations are solved using the same $f_{s}^{*}$ values. Using the no-cylinder model described in Chapter 3, the steady-state reaction rates are calculated and plotted as symbols in Fig. 4.2. Additionally, the analytical solution for the limiting case, Eqn. 4.6, is plotted in Fig. 4.2 as the solid line. Since the analytical solution is only valid for large $f_{s}^{*}$, it cannot be compared with simulation data for smaller $f_{s}^{*}$. The strong agreement for the maximum reaction rate limiting case validates the three methods. For the cases of smaller $f_{s}^{*}$ values, the reaction rate curves diverge below the analytical curve when $f_{s}^{*} \leq \lambda$.

In Fig. 4.2, as $f_{s}^{*}$ decreases, the reaction rate decreases due to insufficient scalar concentrations. The small filament widths diffuse across each other and react away the scalars. As the 
filament width decreases, the reaction rate maximum decreases since more of the reactants are exhausted farther upstream. There is good agreement for the solutions of numerical integration and the simulation further validating the methods.

The other constraint to be relaxed is $\mathrm{Da} \ll 1$, (while maintaining $f_{s}^{*} \gg \lambda$ ). The effect of increasing Da on reaction rate is investigated using the numerical model. Since the ADR equations are coupled when $\mathrm{Da}>0$, the reaction rate cannot be investigated using the analytical solution, nor the numerically integrated solution. In a series of numerical simulations, the reaction rate is solved for $\mathrm{Da}=0.0018,0.18$, and 1.8, and plotted as symbols in Fig. 4.3.

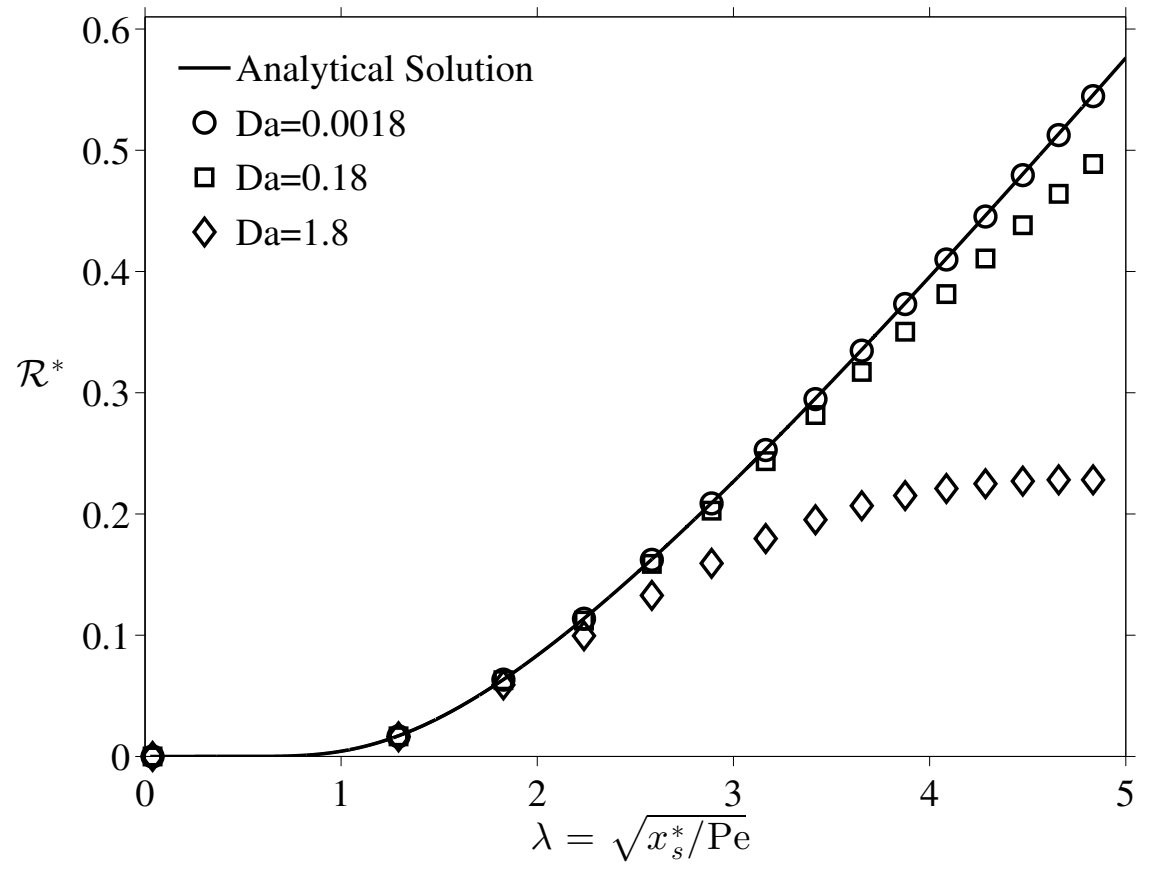

Figure 4.3: Integrated reaction rate results for the no-cylinder case for $\mathrm{Da}=0.0018,0.18$, and 1.8 , where, the analytical solution is the solid line and the symbols are the simulation results.

Damköhler number is the ratio of diffusive and chemical time scales and can be described as the nondimensional reaction rate constant. For $\mathrm{Da} \ll 0$, there is a large quantity of scalars available for reaction which is limited by the reaction rate constant. For $\mathrm{Da} \gg 0$, fast reactions eliminate 
large quantities of scalars, leaving less reactants available. The fast reaction rate will produce more product than with a slower rate, however, production increase is not linear. Fig. 4.3 shows plotted results for reaction scaled by the reaction rate constant, therefore showing the reaction rate due to the fluid mechanics, and not influenced by chemical kinetics. The analytical solution for the limiting case, Eqn. 2.22, is plotted as the solid line in Fig. 4.3. The analytical solution shows the maximum reaction rate for the case without cylinder wake. The simulation solution for Da $=$ 0.002 is a good match to the analytical solution at this range of $\lambda$ values. As Da increases, the reaction rate diverges from the analytical solution farther upstream and then decreases to zero far downstream. 


\section{Chapter 5}

\section{Reactions With Cylinder Wake}

The reactions of separated scalars are investigated in the wake of a cylinder using the numerical model described in Chapter 3. The flow field behind the cylinder is solved using the COMSOL numerical simulation at $\mathrm{Re}=97$. The simulations determine the reaction rate between the two scalars which is investigated for a series of varying parameters. Dimensional analysis (in Chapter 2) determines that there are four parameters that affect the reaction rate between the two scalars, including the separation between the filaments, the width of the filaments, Schmidt number, the Damköhler number. The effect of each parameter is investigated individually by running a series of simulations for a range of a single parameter while holding the three other parameters constant and comparing the resulting reaction rate and reaction product.

Equation 2.21 is not used to plot the reaction rate due to its dependence on $s$. Rather, the dependence on $s$ is removed by laterally integrating the reaction rate over the dimensional coordinate $y$ and phase averaging as

$$
\mathcal{R}(x)=\frac{1}{T_{0}} \int_{0}^{T_{0}} \int_{-\infty}^{\infty} R^{*} \mathrm{~d} y \mathrm{~d} t .
$$

It can be shown that Eqns. 2.21 and 5.1 are related as $\mathcal{R}=s \mathcal{R}^{*}$. Removing the $s$ dependence

allows us to plot the reaction rate for simulations with various separations, but with the effect of the separation removed, only look at the effect of the cylinder wake. The reaction rate is plotted against the downstream length, $x$, scaled by the cylinder diameter, $\phi$; (we do not plot with $\lambda$ as it is also a function of $s$ ). Using these metrics, the reaction data from the simulations can be plotted 
and compared.

\subsection{Effect of Scalar Separation}

Filament separation, $s$, and filament width, $f$, describe the scalar release geometry, where $s^{*}=s / \phi$ and $f^{*}=f / \phi$. A series of simulations are run for varying $s^{*}$ values. Figure 5.1 shows the instantaneous $C_{1}$ and $C_{2}$ scalar concentration fields in the left column, and associated reaction rates in the right column, for simulations with $s^{*}=2$ in the top row and $s^{*}=0.25$ in the bottom row. All other parameters are held constant as $f^{*}=3, \mathrm{Sc}=10$, and $\mathrm{Da}=0.01$. The figure shows that as the separation decreases, the scalars coalesce farther upstream, initiating reaction sooner, resulting in a greater overall reaction rate. The scales for the scalar concentration fields and the reaction rate images are shown in Fig. 5.2.

$$
C_{1} \text { and } C_{2}
$$
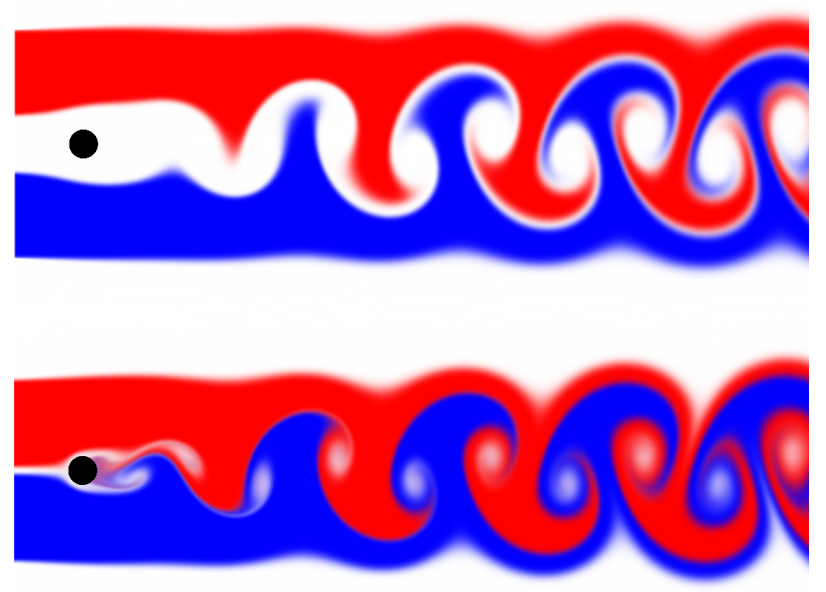

$$
R / k=C_{1} C_{2}
$$
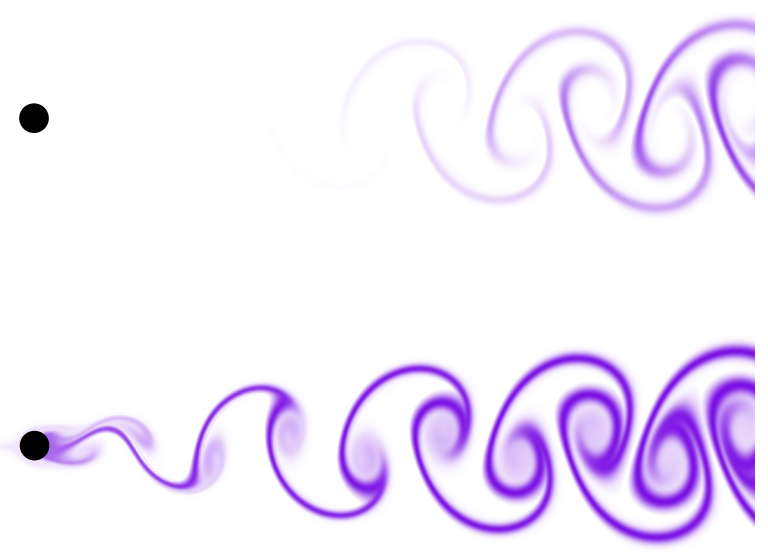

Figure 5.1: Instantaneous scalar concentrations, $C_{1}$ and $C_{2}$ (left column) and associated reaction rate (right column) for large separation, $s^{*}=2$ (top row), and small separation, $s^{*}=0.25$ (bottom row). 

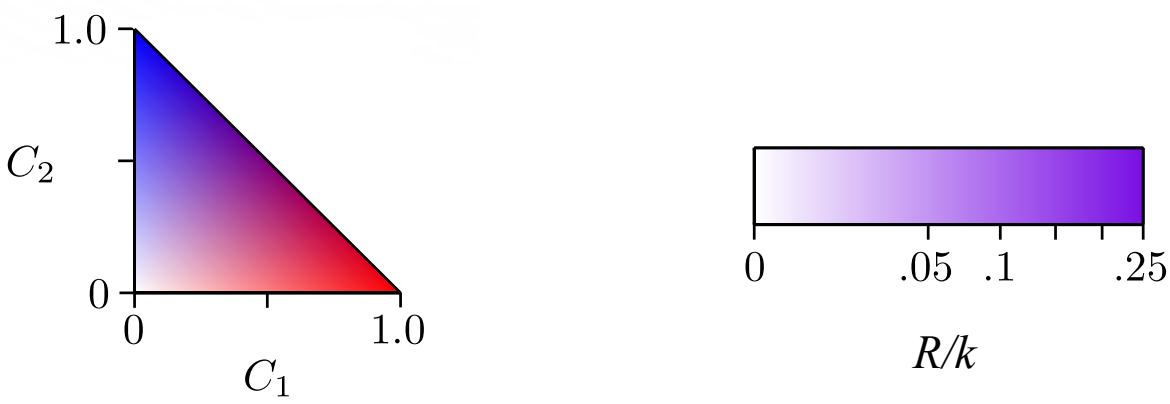

Figure 5.2: Color scale for all scalar concentration fields (left) and logarithmic color scale for all reaction rate images (right).

Using Eqn. 5.1, simulation reaction rates are plotted in Fig. 5.3 for various separation distances. The cylinder is centered at $x / \phi=3.8$. The plot shows the reaction rate results for four separation values, $s^{*}=0,0.25,1$, and 2 . For no separation, $s^{*}=0$, the scalars diffuse and react immediately at the release. For small $s^{*}$ there is a small reaction rate spike just upstream of the cylinder where the scalars momentarily lag in the stagnation streamline. There is a larger spike immediately downstream of the cylinder where the scalars are well mixed in the chaotic region. This is a general feature of all the results. As the vortices shed and advect, the scalars are drawn downstream increasing the interfacial length between the scalars, facilitating diffusion and increasing the reaction rate. As the separation distance is increased, it takes longer for the scalars to coalesce, shown on the plot as the long period of zero reaction rate for the $s^{*}=2$ case. For the separation distances tested, all simulations exhibited the same steady increasing behavior at some distance downstream. As the separation distance increases, it takes longer for the scalars to coalesce which results in lesser reaction rates. 


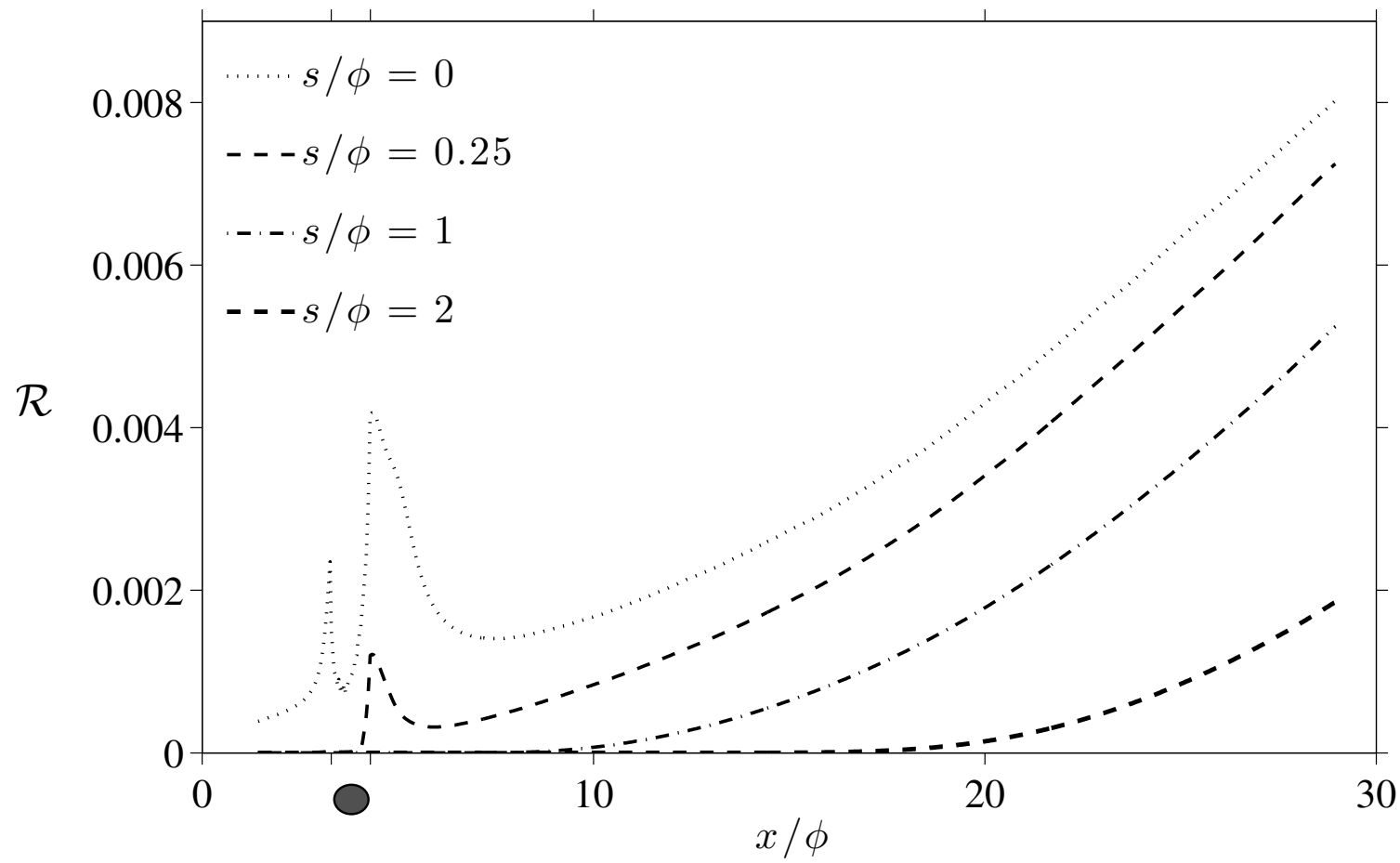

Figure 5.3: Integrated reaction rates for cylinder wake simulations of various scalar separations, where $s^{*}=0,0.25,1,2$ and $f^{*}=3, \mathrm{Sc}=10$, and $\mathrm{Da}=0.01$, plotted with downstream distance scaled by cylinder diameter.

\section{$5.2 \quad$ Effect of Filament Width}

The filament width, $f^{*}$, is varied in a series of simulations to determine the effect on the reaction rate. All other parameters are held constant at $s^{*}=0.25, \mathrm{Sc}=10$, and $\mathrm{Da}=0.01$. Two simulations are compared in Fig. 5.4, showing the instantaneous scalar concentration fields in the left column and the associated reaction rate in the right column, for simulations with $f^{*}=0.25$ in the top row, and the larger $f^{*}=3$ in the bottom row. 

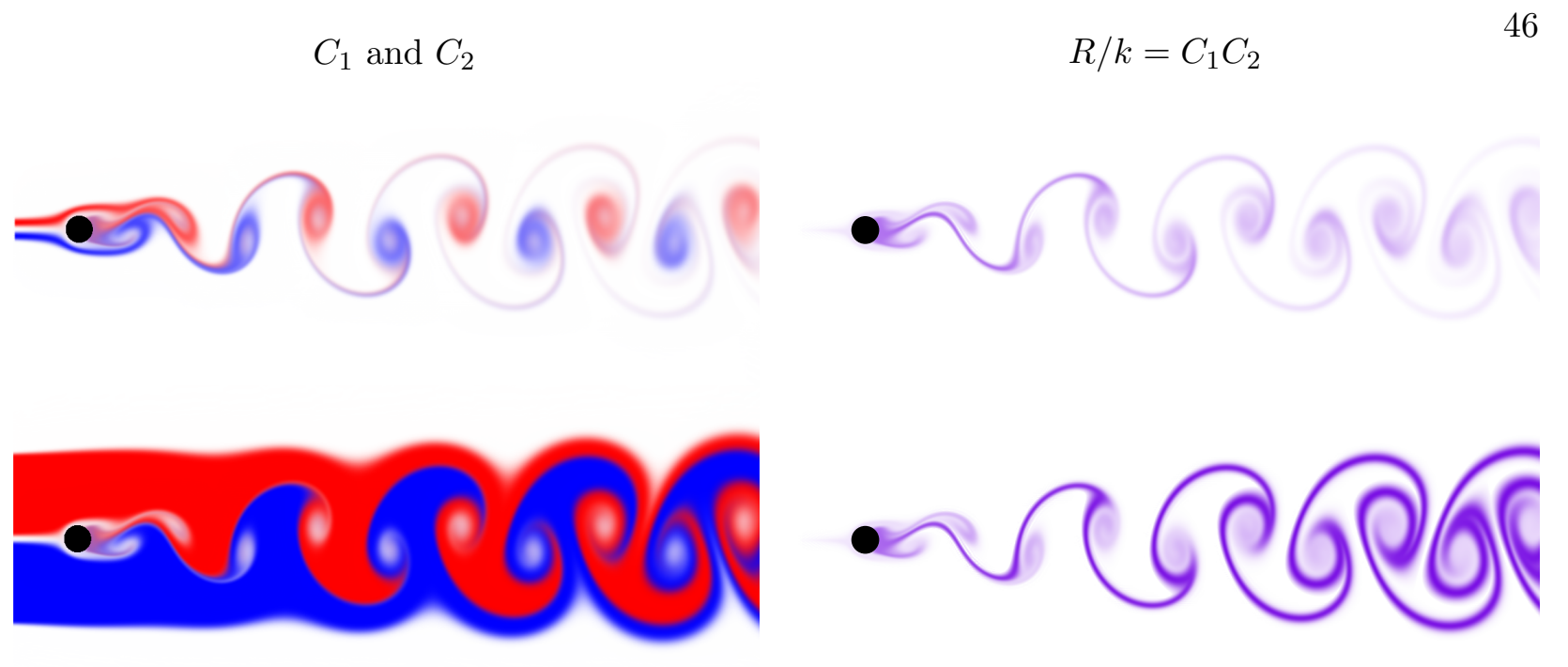

Figure 5.4: Instantaneous scalar concentrations, $C_{1}$ and $C_{2}$ (left column) and associated reaction rate (right column) for the smaller $f^{*}=0.25$ (top row) and larger $f^{*}=3$ (bottom row).

The reaction rate images in Fig. 5.4 show that while reaction does occur for the smaller $f^{*}$ simulation, the reaction rate for the larger $f^{*}$ simulation is more significant, shown by the strong purple color intensity. For the smaller $f^{*}$ simulation, the intensity of the purple color fades downstream illustrating a weaker reaction rate with downstream distance. Eqn. 5.1 is used to quantify the reaction rates for the simulations and is plotted with $x / \phi$ in Fig. 5.5. The cylinder is centered at $x / \phi=3.8$. 


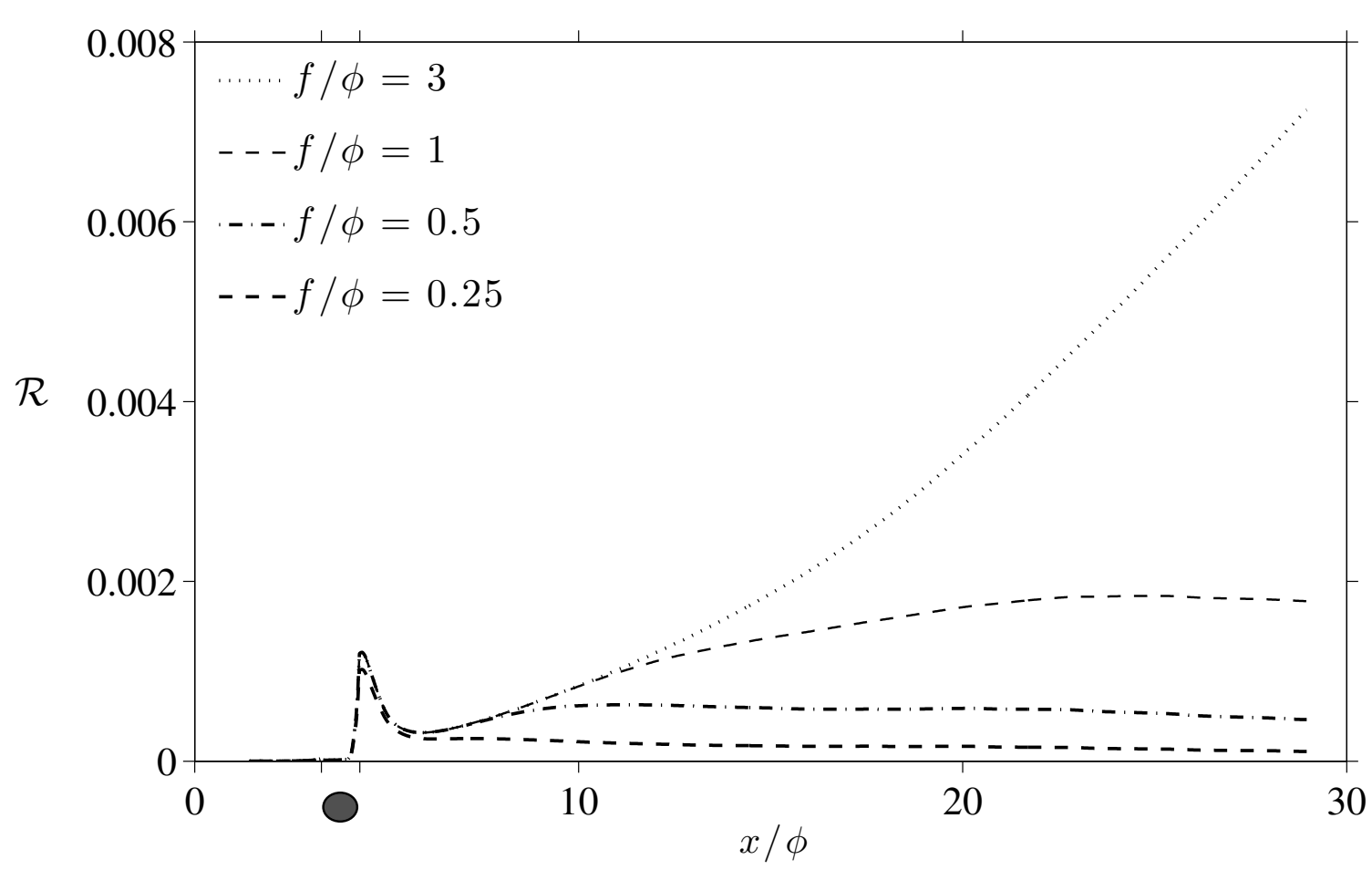

Figure 5.5: Integrated reaction rates of cylinder wake simulations for various scalar filament widths, where $f^{*}=0.25,0.5,1,3$, and $s^{*}=0.25, \mathrm{Sc}=10$, and $\mathrm{Da}=0.01$.

In Fig. 5.5, the integrated reaction rate results are plotted for simulations with $f^{*}=0.25,0.5,1,3$, and $s^{*}=0.25, \mathrm{Sc}=10$, and $\mathrm{Da}=0.01$. Past the chaotic region, scalars advect downstream increasing the interfacial scalar length and facilitating diffusion. In cases of large $f^{*}$, there is an ample supply of scalar to diffuse and react, allowing the reaction rate to continuously increase, being limited only by diffusion. Whereas, in cases of smaller $f^{*}$, a smaller amount of scalar is released into the flow and, although the scalar interface length increases with advection, the reaction rate is limited by insufficient scalar concentrations due to diffusion and reaction that occurs farther upstream. As the filament width increases, the reaction rate increases.

\subsection{Effect of Schmidt Number}

In a series of simulations, the Schmidt number, Sc, is varied while all other parameters are held constant as $s^{*}=0.25, f^{*}=3$, and $\mathrm{Da}=0.01$. The Schmidt number, defined as the ratio 
of momentum diffusion to mass diffusion, describes the diffusive behavior in fluid transport. Two simulation results are compared in Fig. 5.6 showing the instantaneous scalar concentration fields in the left column, and associated reaction rates in the right column, for a model with $\mathrm{Sc}=1$ in the top row, and a model with $\mathrm{Sc}=20$ in the bottom row. The comparison shows that for larger Sc values, the weakly diffusive scalars react only as a thin filament along the scalar interface. However, for smaller Sc values, the scalars diffuse faster and over a broader range, reacting over a wide band across the scalar interface.

$$
C_{1} \text { and } C_{2}
$$

$$
R / k=C_{1} C_{2}
$$
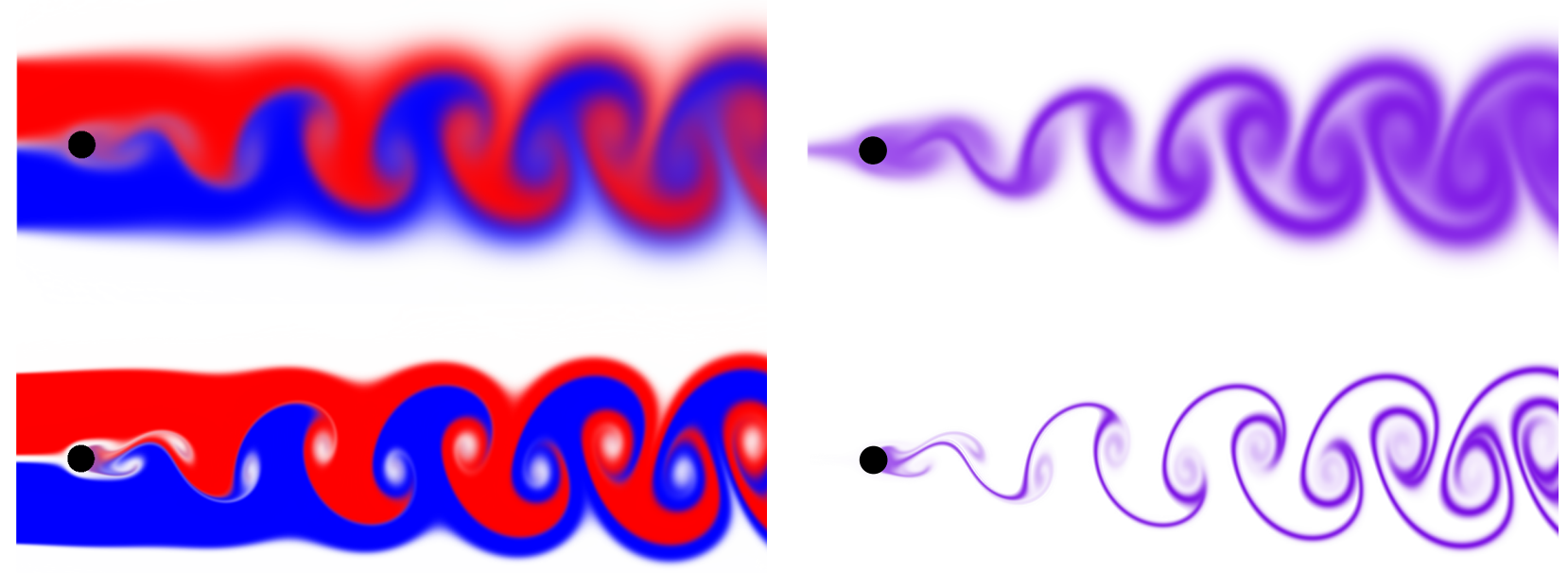

Figure 5.6: Instantaneous scalar concentrations, $C_{1}$ and $C_{2}$ (left column) and associated reaction rate (right column) for $\mathrm{Sc}=1$ (top row) and $\mathrm{Sc}=20$ (bottom row).

Equation 5.1 is used to plot the integrated reaction rate of simulations with varying Sc values in Fig. 5.7. The simulations with larger values of Sc diffuse less along the scalar interface, and although the interface length is increasing with advection downstream, the scalars disperse very little resulting in the mild increase in reaction rate. For smaller values of Sc, the highly diffusive scalars disperse rapidly, overlapping across a relatively wide distance across the interface which promotes the quickly increasing reaction rate. The reaction rate has a small peak upstream of the cylinder for the case of $\mathrm{Sc}=1$, as the highly diffusive scalars react immediately after the release. 


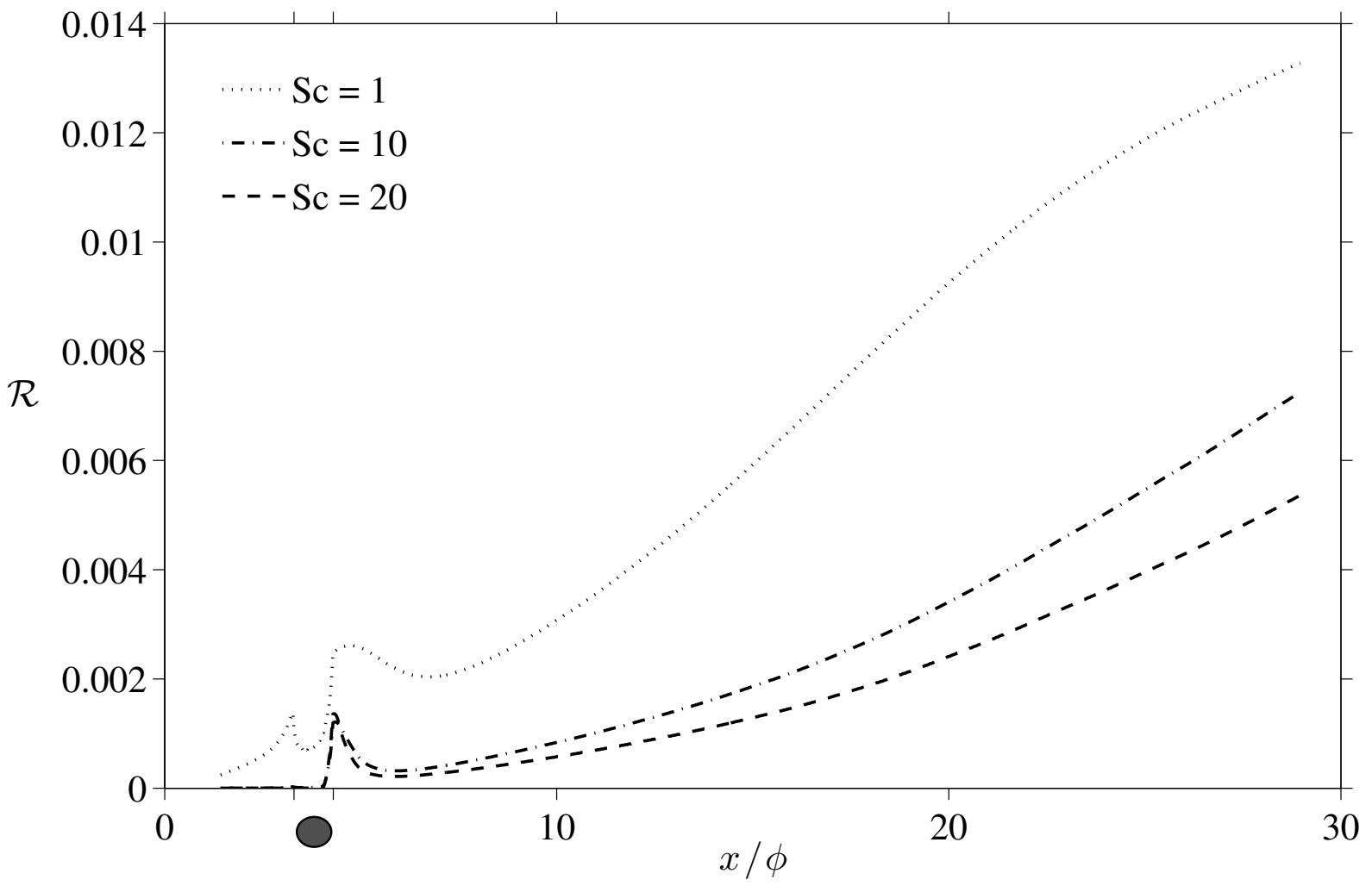

Figure 5.7: Integrated reaction rates for cylinder wake simulations of $\mathrm{Sc}=1,10,20$ with constant $s^{*}=0.25, f^{*}=3$, and $\mathrm{Da}=0.01$ plotted with downstream distance scaled by cylinder diameter.

\subsection{Effect of Damköhler Number}

The Damköhler number is varied in a series of simulations to determine the effects on the reaction rate. The Damköhler number, Da, relates the time scales between diffusion and reaction. A large Da corresponds to a flow in which the scalars react very quickly and the reaction is limited by the speed of the scalar dispersion. Alternatively, a small Da corresponds to a flow with relatively diffusive scalars that disperse over a wide range, but that react slowly. In this case, the reaction is limited by the chemical kinetics. 


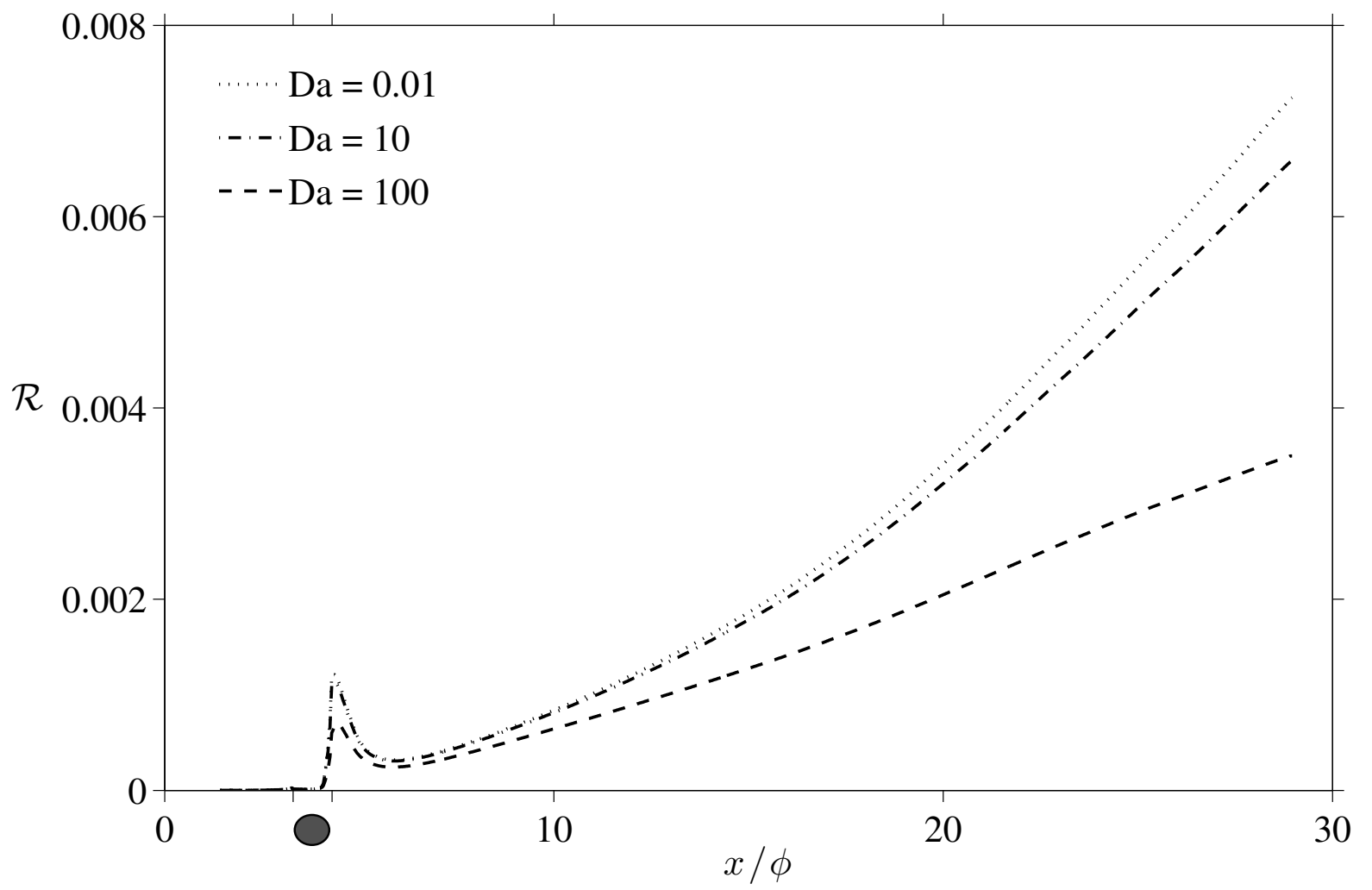

Figure 5.8: Integrated reaction rates for cylinder wake simulations of $\mathrm{Da}=0.01,10$, 100 with constant $s^{*}=0.25, f^{*}=3$, and $\mathrm{Sc}=10$, plotted with downstream distance scaled by cylinder diameter.

In Fig. 5.8, the integrated reaction rates are shown for three simulations of $\mathrm{Da}=0.01,10$, 100 with constant $s^{*}=0.25, f^{*}=3$, and $\mathrm{Sc}=10$, plotted with downstream distance scaled by cylinder diameter. All three models show the general trend of increasing reaction rate after the scalars leave the chaotic region, however, as Da increases, the integrated reaction rate decreases. As the reaction rate constant increases, the reaction product increases, but at a less than linearly rate. Normalizing by the reaction rate constant then reduces the reaction rate more than linearly, therefore the reaction rates plot lower with increasing Da. 


\subsection{Effect of Cylinder Wake}

The reactions from the cylinder model are compared with the reactions from the no-cylinder model. This comparison shows the direct effects that the cylinder wake has on the reaction rates. The filament separation, $s^{*}$, affects the reaction rate in rectilinear flow and in cylinder wake. Figure 5.3 showed the reaction rate results for cylinder wake simulations with various $s^{*}$ values. Figure. 5.9 shows two of those results in addition to reaction rates of the corresponding no-cylinder wake cases, calculated by numerically integrating Eqn. 5.1. Figure 5.9a shows results for $s^{*}=0.25$, and Fig. 5.9b shows results for $s^{*}=1$.
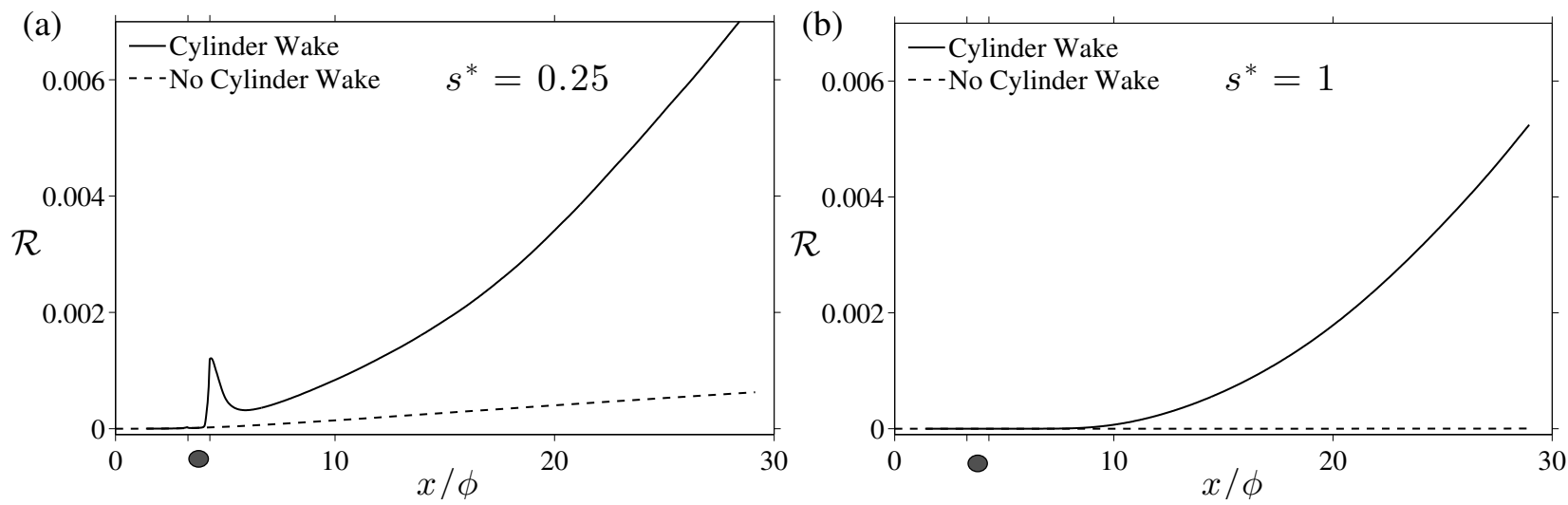

Figure 5.9: Integrated reaction rates for the cylinder wake case and the no-cylinder case for (a) $s^{*}=0.25$ and (b) $s^{*}=1$, while $f^{*}=3, \mathrm{Sc}=10$, and $\mathrm{Da}=0.01$, plotted with downstream distance scaled by cylinder diameter.

In both cases, the reaction rates for the cylinder models increase steeply with downstream distance, within our domain; while the no-cylinder reaction rate ranges from nearly zero to only a mild increase for smaller $s$. In all cases investigated, the cylinder wake reaction rates are significantly larger than reaction rates in the corresponding no-cylinder flow. To determine the reaction rate increase due specifically to the cylinder wake stirring for a range of separation values, the cylinder wake simulation results from Fig. 5.3 are plotted with the analytical solution in Fig. 5.10 on $\log$-log scale. 


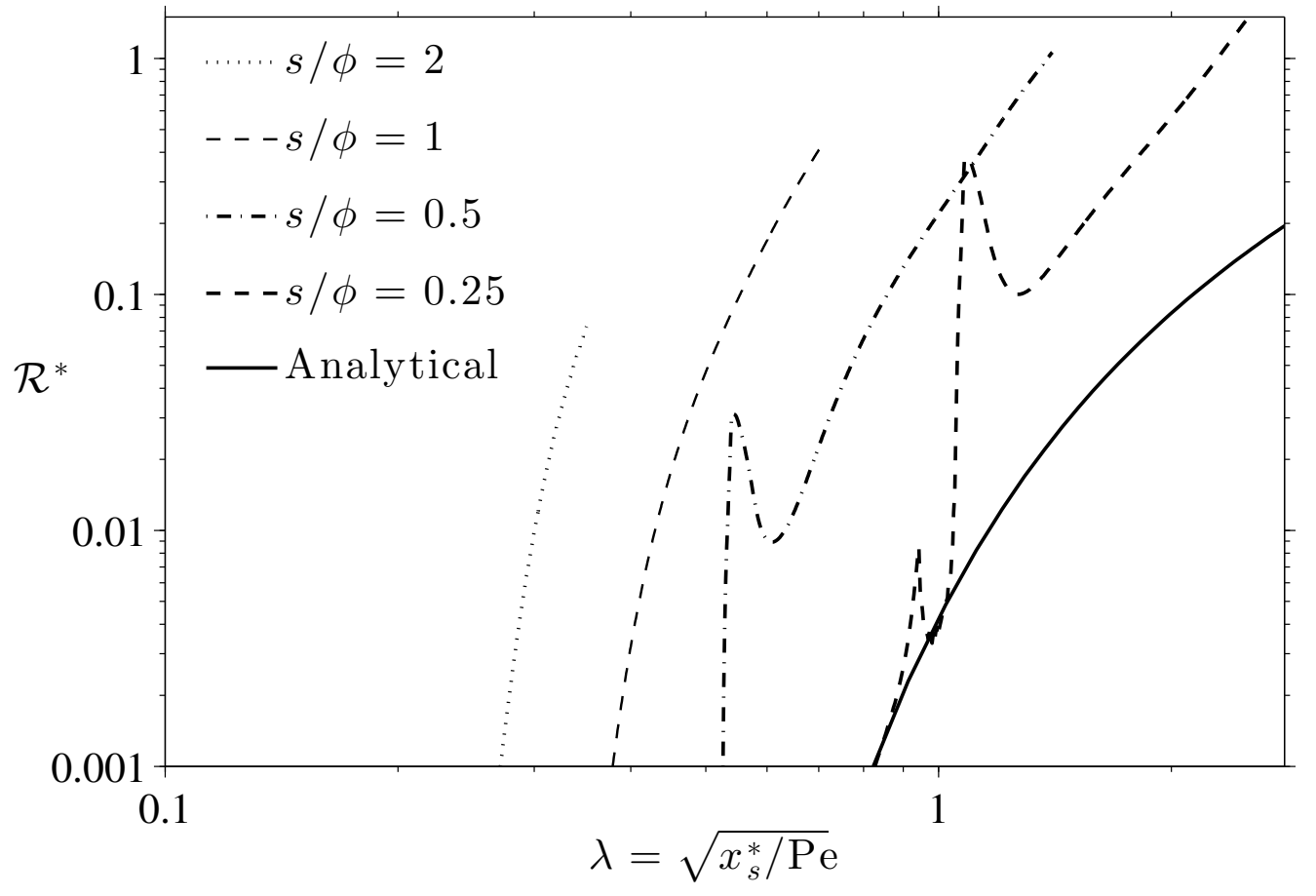

Figure 5.10: Integrated reaction rate results for cylinder models with various values of $s / \phi$, compared to the analytical solution for no-cylinder wake. Broken lines are results from the cylinder model, solid line is for the no-cylinder model.

Recall that the analytical solution, Eqn. 4.6, was normalized by the separation parameter and therefore the curves for all $s^{*}$ values collapse onto a single curve, removing the effect of the separation. So then, Fig. 5.10 shows the reaction rate increase due strictly to the cylinder wake for simulations with various $s^{*}$ values. For simulations with increasing $s^{*}$, the cylinder wake stirring has a more substantial role in bringing the scalars together and produces reaction rates that are significantly larger than the no cylinder case, particularly for cases of large $s^{*}$.

The filament width, $f^{*}$, affects the reaction rate in both rectilinear flow and in cylinder wake. Figure 5.11a shows reaction rate results for a specific cylinder wake simulation directly compared to results from the corresponding no-cylinder simulation, where both models have the same parameters: $s^{*}=0.25, f^{*}=1, \mathrm{Sc}=10$, and $\mathrm{Da}=0.01$. Figure. $5.11 \mathrm{~b}$ shows the comparison between the cylinder and no-cylinder simulations with the filament width changed to $f^{*}=3$, while 
the other parameters remain constant as $s^{*}=0.25, \mathrm{Sc}=10$, and $\mathrm{Da}=0.01$.
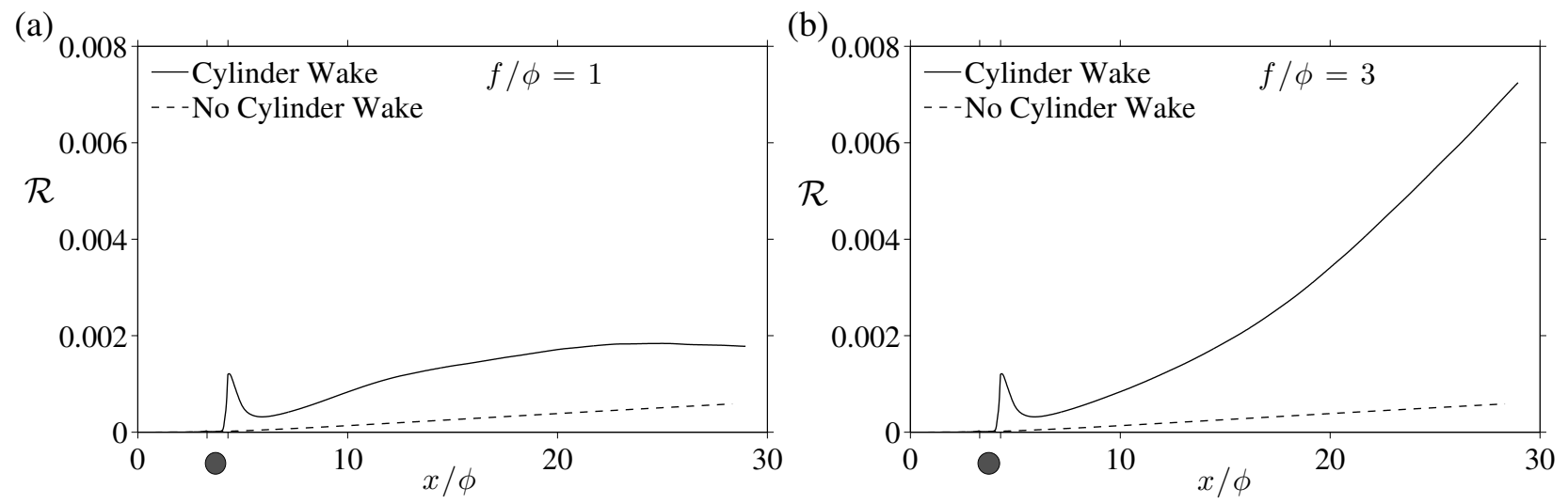

Figure 5.11: Reaction rates for the cylinder wake case and the no-cylinder case for (a) $f^{*}=1$ and (b) $f^{*}=3$, while $s^{*}=0.25, \mathrm{Sc}=10$, and $\mathrm{Da}=0.01$, plotted with downstream distance scaled by cylinder diameter.

In both plots of Fig. 5.11, the no-cylinder wake results show a mild reaction rate increase with downstream distance. The cylinder wake reaction rates for the $f^{*}=1$ case are a bit greater than the no-cylinder reaction rates. In this case, the cylinder wake reaction rates (downstream of the chaotic region spike) slowly increases to a peak, and then decreases. Whereas, in the $f^{*}=3$ case, the reaction rates steadily continue to increase with downstream distance. A wider filament width allows the structured wake to be taken advantage of which facilitates and promotes reactions to occur at the maximum possible reaction rates. Both plots shows that the reaction rates are much greater in the cylinder wake flows than in the rectilinear flow.

A series of simulations were run to investigate the effects of the cylinder wake at various Schmidt number, Sc. In the simulations, $\mathrm{Sc}$ is varied as $\mathrm{Sc}=1,10$, and, 20, and all other parameters are held constant as $s^{*}=0.25, f^{*}=3$, and $\mathrm{Da}=0.01$. The resulting integrated reaction rates were plotted in Fig. 5.7 on a linear scale. The same results are plotted on log-log scale in Fig. 5.12. The analytical solution, Eqn. 2.21, plotted as the solid line, can represent the maximum possible reaction rate for the no-cylinder case. The simulation reaction rates for all Sc values investigated are larger than the analytical solution. Furthermore, as Sc decreases, scalar behavior is more diffusive 
and reaction rates increase. For very small Sc, the increase can be over orders of magnitude. A large reaction rate increase is shown in the $\mathrm{Sc}=1$ case, where reaction occurs even upstream of the cylinder.

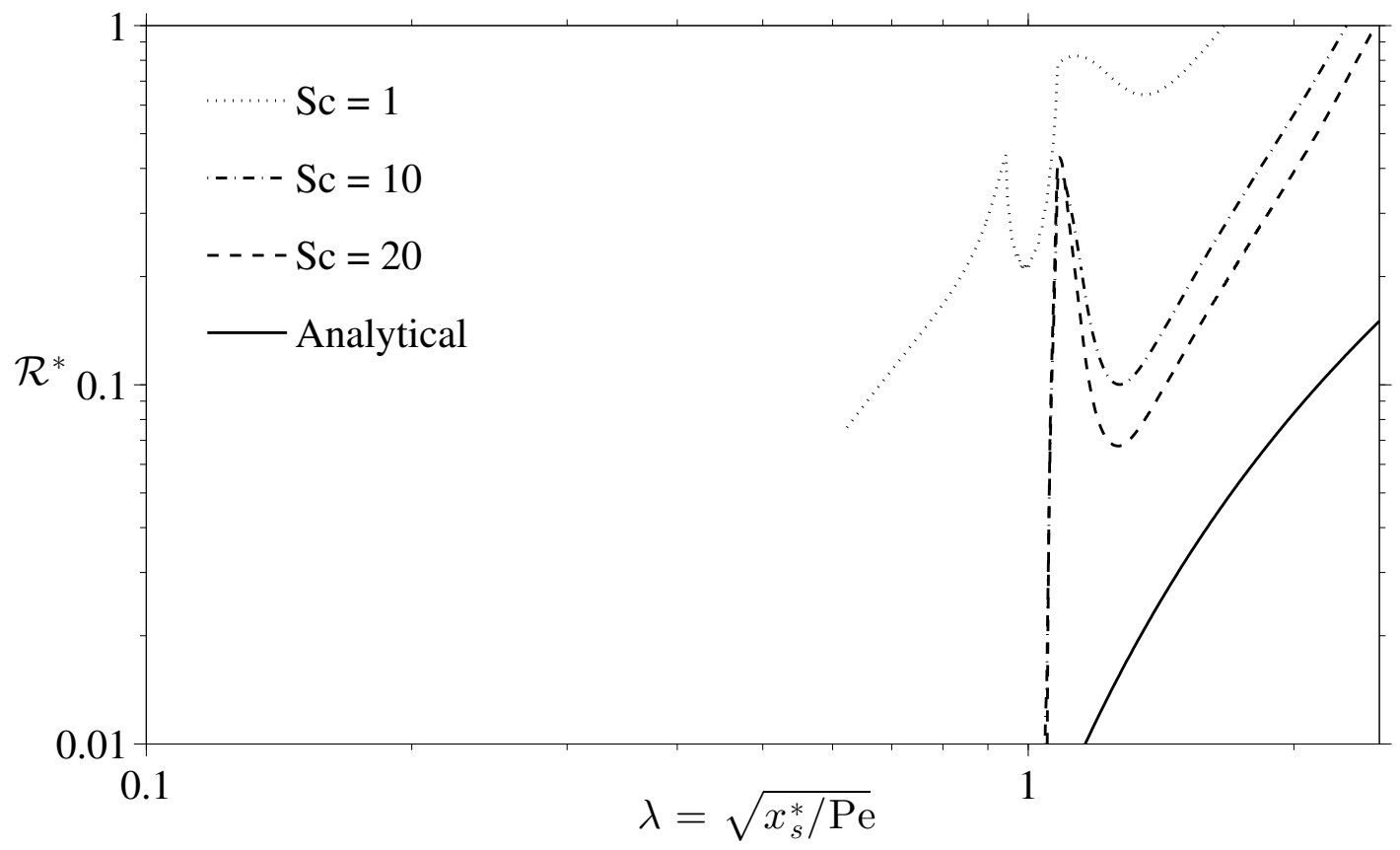

Figure 5.12: Integrated reaction rate results for cylinder models with various values of Sc, compared to the analytical solution for no-cylinder wake. Broken lines are results from the cylinder model, solid line is for the no-cylinder model.

Finally, simulations were run to investigate the effects of cylinder wake at various Damköhler numbers. Simulation reaction rate results are shown in Fig. 5.13 for two cases, (a) Da $=10$, and (b) $\mathrm{Da}=100$, while all other parameters are held constant as $s^{*}=0.25, f^{*}=3$, and $\mathrm{Sc}=10$. For comparison, reaction rates are plotted for the cylinder wake case as solid lines and the no-cylinder wake case as dashed lines. The plot shows that as Da decreases the reaction rate increases in both the cylinder wake case and in the no-cylinder wake case. However, the reaction rate in the cylinder wake increases more than in the no-cylinder wake at some local downstream location. Cylinder wake stirring has an increasingly significant impact on the reaction rate as Da decreases. 
(a)

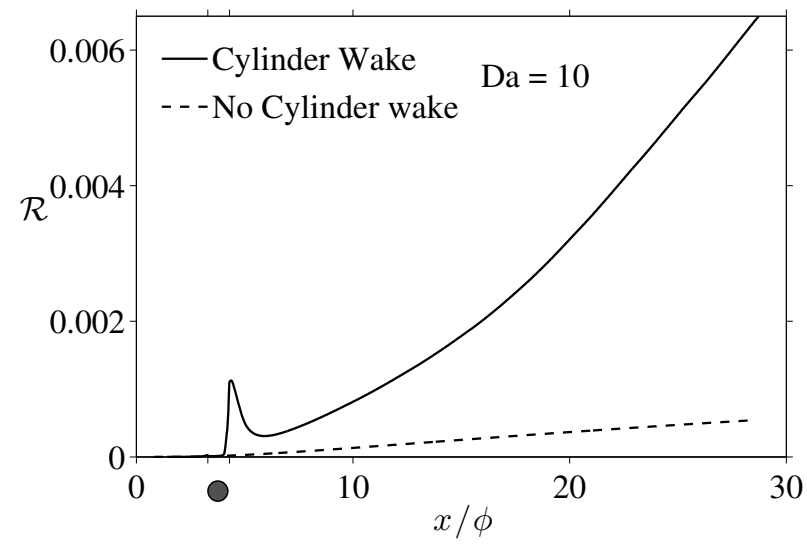

(b)

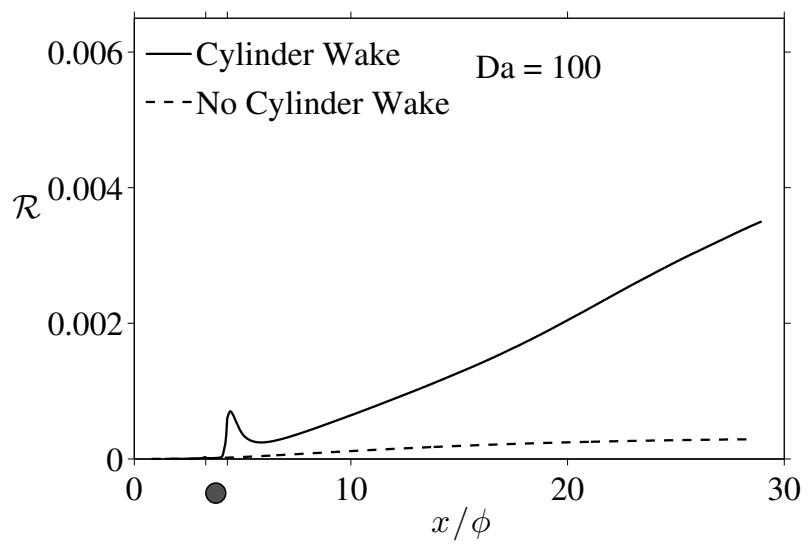

Figure 5.13: Reaction rates for the cylinder wake case and the no-cylinder case for (a) Da $=10$ and (b) Da $=100$, while $s^{*}=0.25, f^{*}=3$, and $\mathrm{Sc}=10$, plotted with downstream distance scaled by cylinder diameter.

\subsubsection{Exception Case}

The overwhelming majority of the results have shown that reaction rates of initially separated scalars dramatically increases in the structured wake of a cylinder when compared to a wake without a cylinder. However, in one scenario, we found that the presence of the cylinder actually inhibits coalescence and produces lower reaction rates than in the wake without the cylinder. This reduced reaction rate occurs with a scalar release geometry that has a small filament separation, $s^{*}$, simultaneously with a small filament width, $f^{*}$. A small filament width will cause the braid region at the scalar interface to disperse and react away relatively quickly. A small separation allows the scalars to be drawn into the chaotic region where vortices are formed and develop with scalar concentrations in the vortex cores, the vortices then shed and advect downstream. In the vortex cores, scalars are sequestered and unable to mix. When both small $s^{*}$ and small $f^{*}$ exist simultaneously, reaction rates peak at early times and steadily decrease. The braid regions react away quickly causing reaction to decrease while, isolated in vortex cores, the remaining scalars are prevented from mixing, causing the reaction rates to decrease further.

Figure 5.14 shows, for two simulations, the instantaneous $C_{1}$ and $C_{2}$ scalar fields in the left 
column and the associated instantaneous reaction rates in the right column. The model shown in the top row has a large filament separation and large filament width $\left(s^{*}=2\right.$ and $\left.f^{*}=3\right)$, while the model in the bottom row has a small separation and small filament width $\left(s^{*}=0.25\right.$ and $f^{*}=0.25$ ). The model with the larger release geometry (top row) has no scalars in the vortex cores, illustrated as the empty white circles, both in the scalar field and in the reaction rate images. In the reaction rate image, the color of the braid region intensifies showing that the reaction rates increase with downstream distance. In the simulation with the smaller release geometry (bottom row), a significant amount of scalars are caught in vortex cores, shown as the blue and red circles in the scalar field. In the reaction rate image, the braid region between at the scalar interface fades downstream showing that the reaction rates are decreasing with downstream distance.

$$
C_{1} \text { and } C_{2}
$$
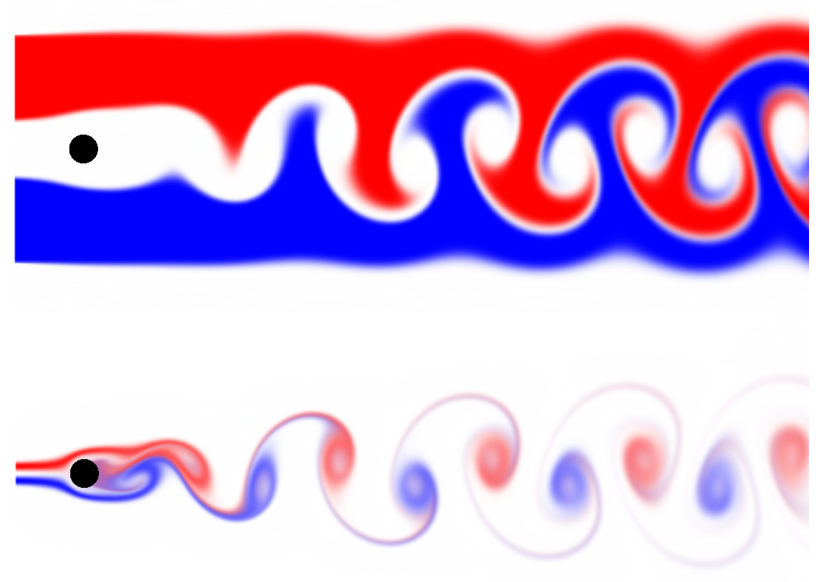

$$
R / k=C_{1} C_{2}
$$
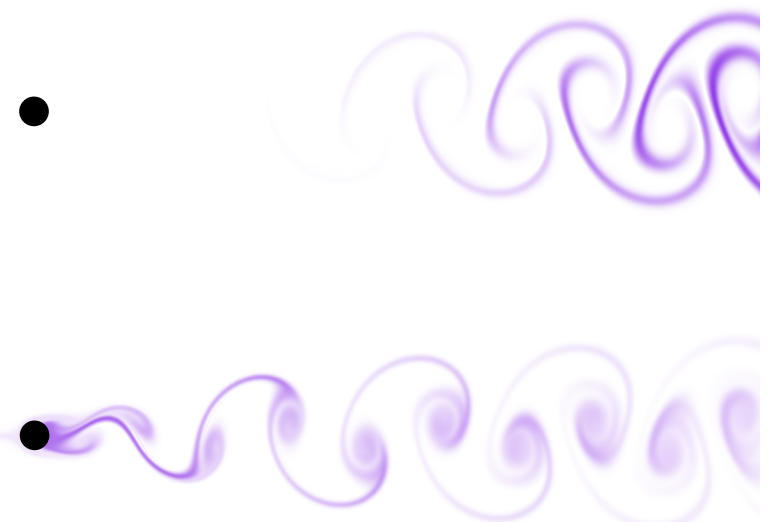

Figure 5.14: Instantaneous scalar concentrations, $C_{1}$ and $C_{2}$ (left column) and associated reaction rate (right column) for large release geometry of $s^{*}=2$ and $f^{*}=3$ (top row) and small release geometry of $s^{*}=0.25$ and $f^{*}=0.25$ (bottom row).

The reaction rates for the cylinder model with a small release geometry are directly compared with the reaction rates for the corresponding no-cylinder model. Figure 5.15a shows the reaction rate results for models with $s^{*}=0.25, f^{*}=0.25, \mathrm{Sc}=10$, and $\mathrm{Da}=0.01$, where the cylinder 
model is plotted as a solid line, and the no-cylinder model is plotted as a dashed line. Figure 5.15b shows similarly, the cylinder to no-cylinder comparison for models with a slightly larger $f^{*}=0.5$.

(a)

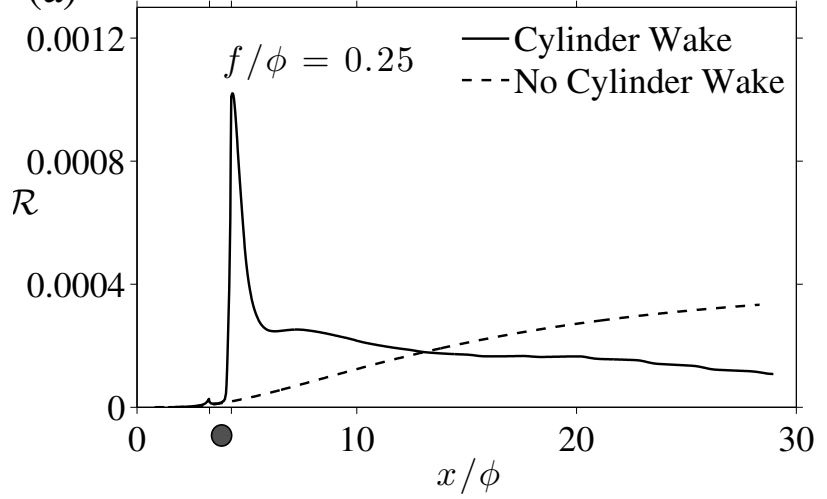

(b)

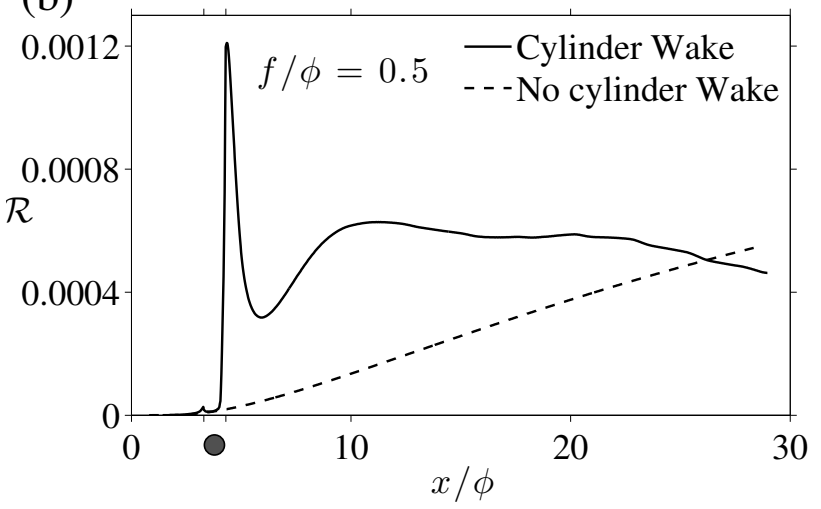

Figure 5.15: Reaction rates for the cylinder wake case and the no-cylinder case for simulations with (a) $f^{*}=0.25$ and (b) $f^{*}=0.5$ while $s^{*}=0.25, \mathrm{Sc}=10$, and $\mathrm{Da}=0.01$, plotted with downstream distance scaled by cylinder diameter.

Both plots show, for the cylinder wake results, a large spike in reaction rate immediately downstream of the cylinder where scalars are mixing in the chaotic region. In Fig. 5.15a, downstream of the chaotic region, the cylinder wake reaction rate tapers down towards zero as the majority of the scalars have already reacted, whereas, the no-cylinder reaction results rise from zero as the scalars diffuse and begin to react. The reaction behavior is similar in Fig. 5.15b, where the reactions rates for the cylinder case drop below the no-cylinder reaction rates. In both cases, having a small $s^{*}$ and small $f^{*}$ release geometry, the local downstream reaction rate for the no-cylinder case exceeds the reaction rates for the cylinder wake case.

\subsubsection{Total Product}

To further quantify the reaction between the initially separated scalars, we calculate the total product the reaction produces in each simulation. The total product produced is equal to the amount of scalar that is reacted out. To keep track of the $C_{1}$ concentration, we use the $C_{1}$ flux. Since, for all cases, the release geometry and downstream behavior is symmetric, we can use the 
product of the $C_{1}$ concentration and the $x$-component of the velocity to calculate the flux. The product of the concentration and the $x$-direction velocity is laterally integrated and phase averaged giving the $C_{1}$ flux across an $x$-location per unit width out of the page. Normalizing by the $C_{1}$ inlet flux, the integrated flux is plotted in Fig. 5.16 with downstream location scaled by cylinder diameter. Normalizing by the inlet flux gives the initial flux a value of unity.

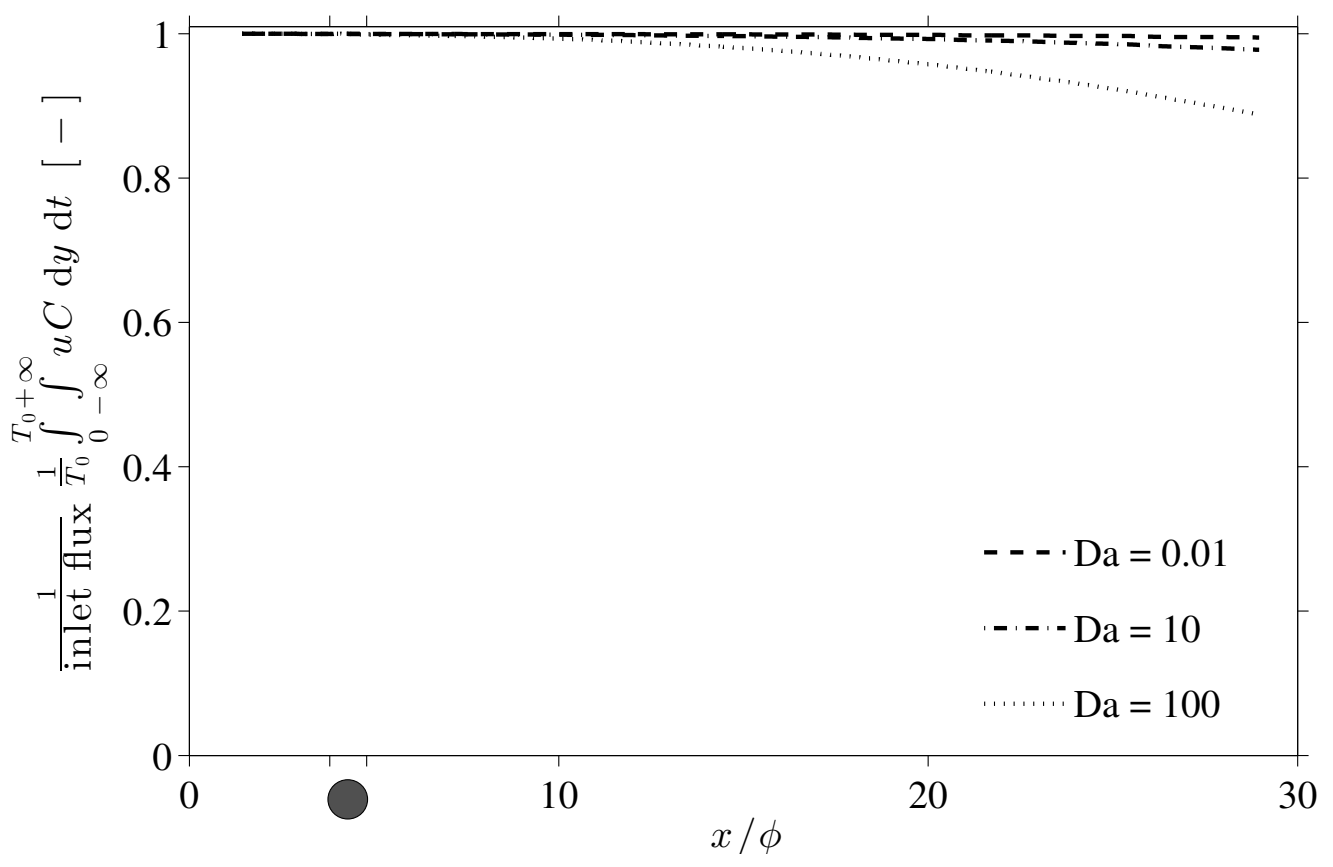

Figure 5.16: Normalized $C_{1}$ flux for models with various Da values. The plot shows the $C_{1}$ mass that passes across an $x$-location per unit width out of the page per unit time.

The normalized $C_{1}$ flux plot illustrates the flux behavior for models with various Da, and with constant $s^{*}=0.25, f^{*}=3$, and $\mathrm{Sc}=10$. Moving downstream, the flux decreases as the scalars react decreasing the mass of $C_{1}$ (and $C_{2}$, equally) in the flow. As the $C_{1}$ scalar reacts, it is eliminated, while, the product $R$ is created. Subtracting the $C_{1}$ flux from the normalized inlet condition (unity), gives the product flux as a percent of the initial flux, that crosses an $x$-location. The total product flux for models with various Da are plotted in Fig. 5.17 with downstream location scaled by the diameter. The total product at the release location is zero for all models, as the scalars have not 
coalesced and reacted. Downstream, the scalars coalesce in the structured wake and begin to react. Farther downstream, the scalar interface is stretched increasing reaction rates, which increases the total product flux. Furthermore, as Da increases the scalars become more diffusive facilitating and promoting reactions (and thus product) to occur more rapidly. Integrating the product flux curve, would give the mass of product that is produced in the domain per time.

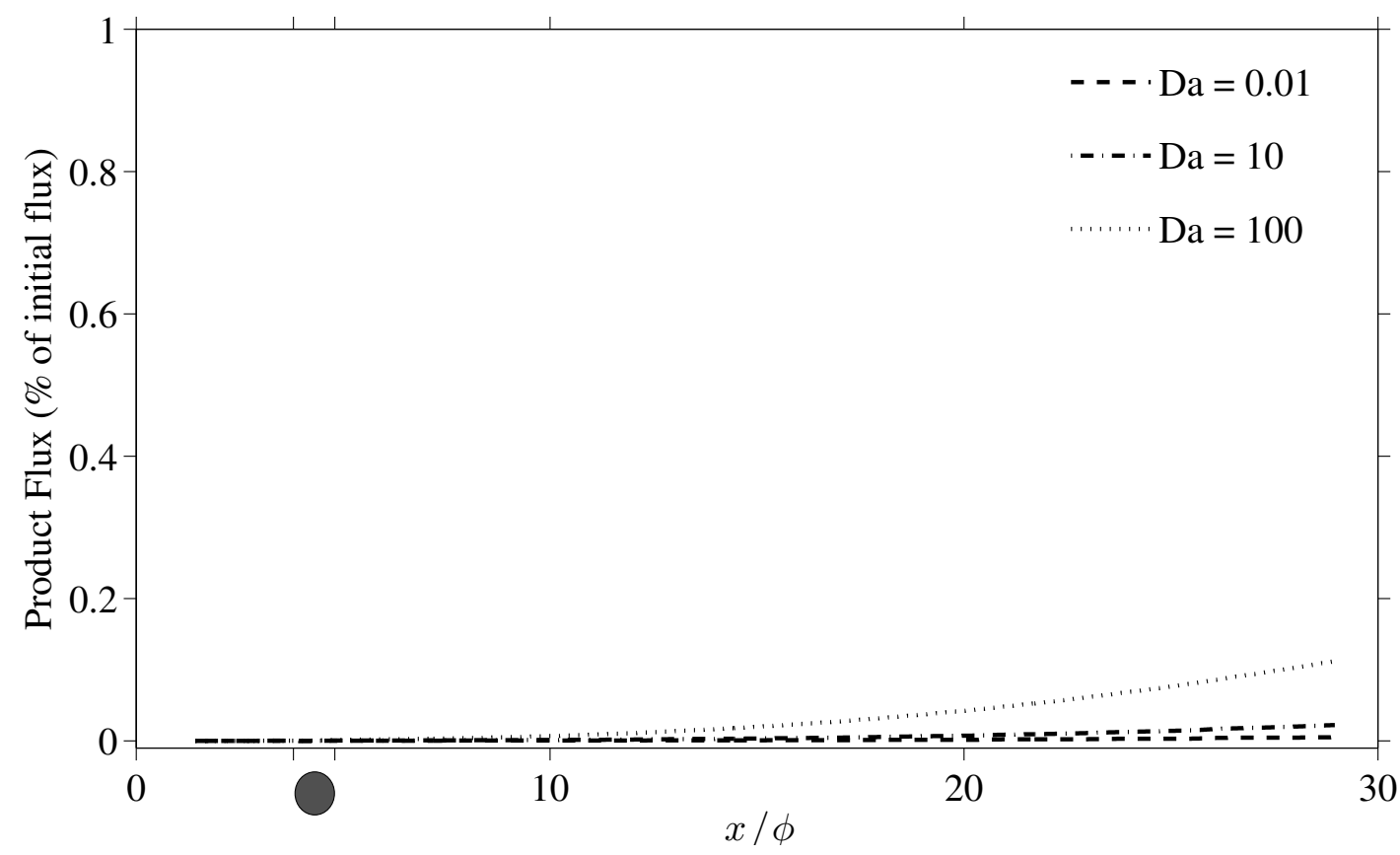

Figure 5.17: Total product flux for models with various values of Da plotted with downstream distance scaled by cylinder diameter. The plot shows the mass of product that crosses an $x$-location per unit width out of the page per unit time. 


\section{Chapter 6}

\section{Conclusions}

In this study, reaction rates of initially distant scalars in a cylinder wake were investigated as four parameters were varied, including, scalar filament separation distance, scalar filament width, Schmidt number, and Damköhler number. These parameters were investigated individually in a cylinder wake and in rectilinear flow. Rectilinear flow develops in a system with no cylinder to disrupt the flow, and is used as a baseline case in cylinder wake comparisons.

\subsection{No-Cylinder Case}

The no-cylinder case is modeled as a relatively simple advection-diffusion-reaction problem, where the reaction between two scalars is solved by numerical integration, Eqn. 2.21. In the limiting case of $f_{s}^{*} \gg \lambda$ and Da $\ll 1$, the numerically integrated solution can be solved analytically, Eqn. 4.6, as a function of the single parameter, $\lambda$, where $\lambda=\sqrt{\left(x_{s}^{*}\right) / \mathrm{Pe}}$. For $\lambda<1$, there is no reaction, as the scalars have not yet diffused across the full separation distance. For $\lambda \gg 1$, reaction scales linearly with $\lambda$, as $(8 \pi)^{-1 / 2} \lambda$.

The effect of relaxing the two analytical constraints had similar results. For $f_{s}^{*}<\lambda$, the reaction rate becomes limited by the amount of scalar in the flow such that the reaction rate decreases with decreasing $f_{s}^{*}$. For $\mathrm{Da}>1$, the increased reaction constant increases the reaction product but at a less than linear rate, and so as Da increases, the reaction rate decreases. 


\subsection{Cylinder Case}

The cylinder wake case models the transport of two initially distant scalars in a complex flow field over a range of parameters. In the cylinder model, as the scalar filament separation increases, the reaction rate decreases, as it takes longer for the scalars to coalesce. Very small filament widths limit the amount of scalars released into the flow which limit the reaction rate, and as filament width increases, the reaction rate increases. Large Schmidt numbers correspond to very weak diffusivities, and as Schmidt number increases, the reaction rate decreases. As Damköhler number increases, the reaction rate decreases due to the less than linear relationship between the reaction rate constant and reaction product.

The resulting reaction rates of the cylinder case are compared to the reactions rates of the nocylinder case over a range of each parameter. It is found that cylinder wake significantly increases reaction rate in the significant majority of cases; particularly for cases of large scalar separation, where the reaction rate increased over orders of magnitude. This shows that cylinder wake is an effective mode stirring that enhances mixing and reaction between two initially distant scalars. In one scenario, it is found the that the presence of the cylinder and associated wake actually inhibit the coalescence of the scalars, and produce lower reactions rates when compared to the reaction rates in the no-cylinder case.

One implication of this study is that structured flow is a mechanism that facilitates successful coral reproduction. Coral heads and other benthic topology on a reef add structure to the ambient flow as water moves around the solid body. In the reproduction strategy of broadcast spawning, male and female corals release gametes into the ambient water and rely primarily on the flow behavior to bring the gametes together to fertilize. This study suggests that the structured flow created due to the presence of a coral head can dramatically increase the fertilization rate of neighboring broadcast spawning corals by orders of magnitude. 


\subsection{Future Work}

\subsubsection{Discrete Release}

This work could be continued by expanding the scalar release parameters, such as releasing the scalar concentrations as a discrete mass, rather than at a continuous rate. In this case, there would only be an advective timescale, as the discrete mass is transported downstream. Quasi-steady-state will not be obtained. Furthermore, since there is a finite volume of scalar in the system, it may be advantageous to observe the reactions in a longer domain to allow a more comprehensive transport investigation.

\subsubsection{Asymmetrical Release}

Another scenario that should be considered is the many cases of an asymmetrical release geometry, where the scalar concentrations are released into the flow asymmetrically about the centerline. The magnitude of the asymmetry is defined as the eccentricity, where $e$ is the distance, in terms of cylinder diameters, from the stagnation streamline to the center of the scalars as shown in Fig. 6.1.

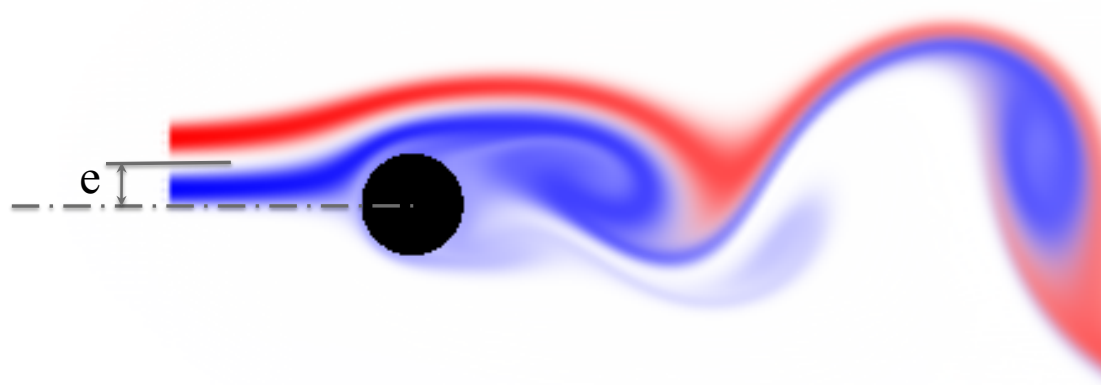

Figure 6.1: Eccentricity, $e$, is the distance, in terms of cylinder diameters, from the stagnation streamline to the centerline between the scalar concentrations at the release. 


\subsubsection{Asymmetrical Investigation 1}

Two asymmetrical cases are investigated. The first case is a model with the small release geometry, where $s^{*}=0.25, f^{*}=0.25, \mathrm{Sc}=10$, and $\mathrm{Da}=0.01$, and an eccentricity of 0.4 cylinder diameters is applied to the release geometry. The resulting $C_{1}$ and $C_{2}$ scalar concentrations are shown in Fig. 6.2 on the left, and the corresponding reaction rate shown on the right. In this case, the release geometry is such that both scalars are released on the same side of the cylinder and the majority of the scalars are drawn around the same side of the cylinder. This causes only the $C_{2}$ concentration to be drawn into the chaotic region and ultimately islands of $C_{2}$ form in the cores of the vortices limiting the reaction rate.

$C_{1}$ and $C_{2}$

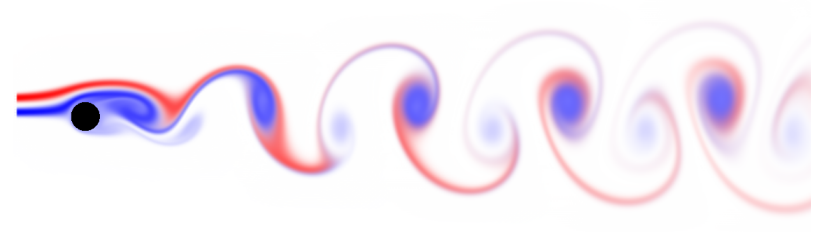

$R / k=C_{1} C_{2}$

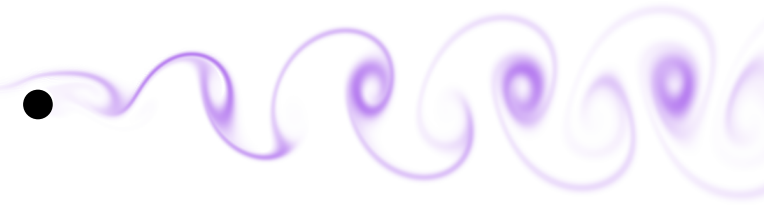

Figure 6.2: Scalar concentrations, $C_{1}$ and $C_{2}$, shown for an asymmetric release geometry (left) where $e=0.4$ cylinder diameters, and the corresponding reaction rate between the $C_{1}$ and $C_{2}$ scalar concentrations (right).

The reaction rate for this asymmetrical case is calculated and compared with the reaction rate for the symmetrical case with the same parameters. The reaction rate is calculated as the laterally integrated phase averaged reaction over one shedding period and is plotted with downstream location scaled by cylinder diameter, shown in Fig. 6.3. The plot shows a steep increase in reaction immediately downstream of the chaotic region, where the scalars are pushed together. The reaction then levels off as the sequestered $C_{2}$ concentration dissipates from the core of the vortices and mixes with the $C_{1}$ concentration. Both cylinder models, symmetric and asymmetric, begin with a higher reaction rate but then fall below the no-cylinder reaction as the concentrations 
deplete downstream.

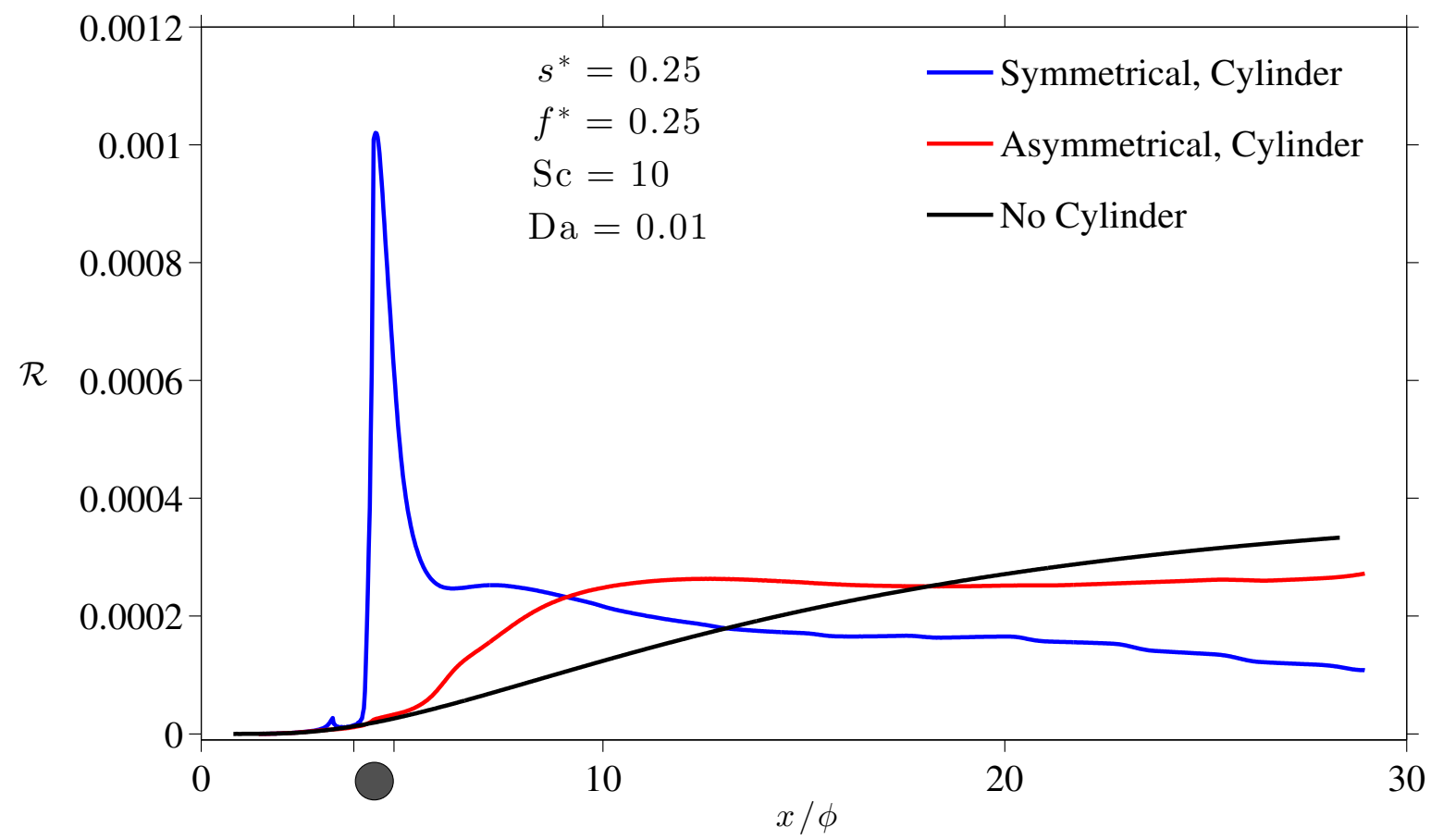

Figure 6.3: Laterally integrated phase averaged reaction rate for a cylinder model with small release geometry, comparing the symmetrical and asymmetrical release geometries, and the no-cylinder case. 


\subsubsection{Asymmetrical Investigation 2}

The second case investigated was for a model with a larger filament width, where $s^{*}=0.25$, $f^{*}=3, \mathrm{Sc}=10, \mathrm{Da}=0.01$, and $e=0.4$ cylinder diameters. The resulting $C_{1}$ and $C_{2}$ concentration field is show in Fig. 6.4 on the left. In this case, since the the filament width is so large, the majority of the $C_{1}$ and $C_{2}$ concentrations still flow around opposite sides of the cylinder. The resulting reaction rate is shown in Fig. 6.4 on the right.

$$
C_{1} \text { and } C_{2}
$$

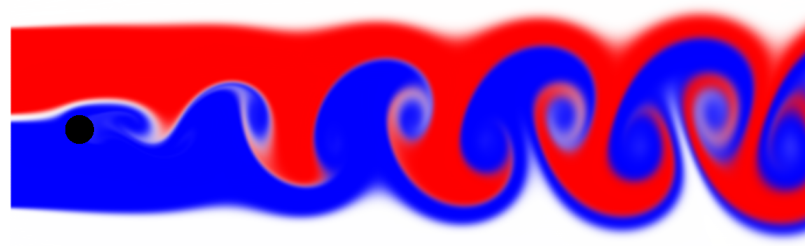

$$
R / k=C_{1} C_{2}
$$

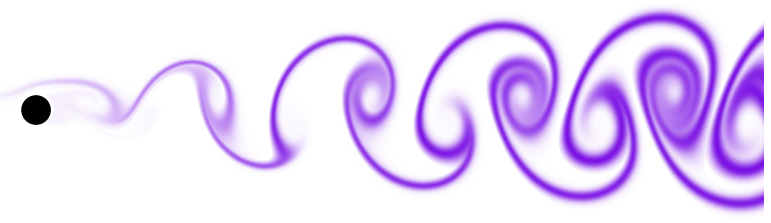

Figure 6.4: Scalar concentrations, $C_{1}$ and $C_{2}$, shown for an asymmetric release geometry (left) where $e=0.4$ cylinder diameters and the corresponding reaction rate between the $C_{1}$ and $C_{2}$ scalar concentrations (right).

The reaction rates were calculated by laterally integrating and phase averaging the instantaneous reaction rates. The reaction rates are compared for both cylinder cases, symmetrical and asymmetrical, and the no-cylinder case and plotted with downstream location in Fig. 6.5. Both the symmetrical and asymmetrical produce reaction rates on the order of magnitudes higher than the case without the cylinder. 


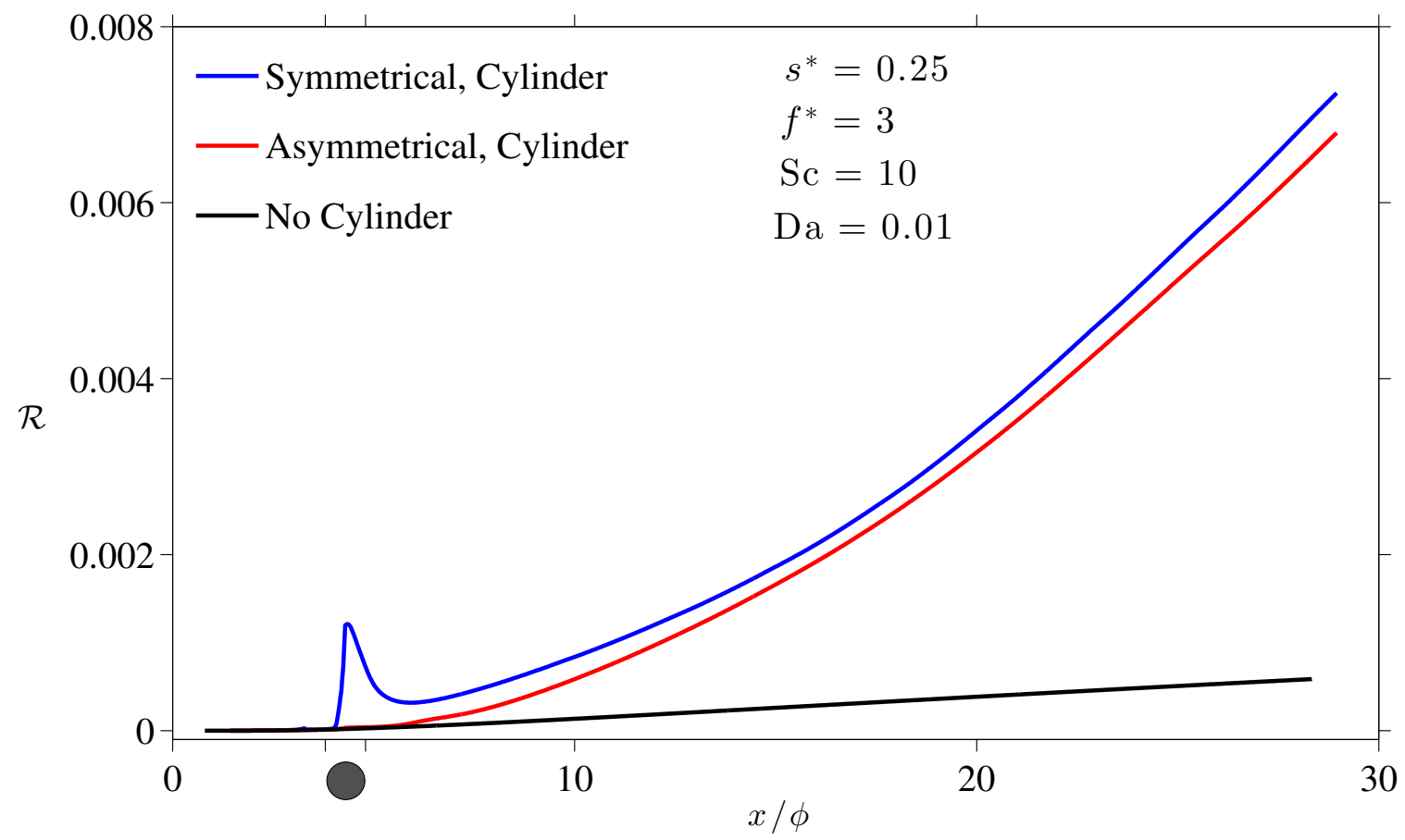

Figure 6.5: Laterally integrated phase averaged reaction rate for a cylinder model with $s^{*}=0.25$ and $f^{*}=3$, comparing the symmetrical and asymmetrical release geometries, and the no-cylinder case.

We've shown in the briefest sense, that our results still hold true for the less-idealized scenario of having an asymmetric release geometry. With an asymmetrical scalar release geometry, the presence of the cylinder increases the reaction rate on the order of magnitudes higher when compared to the same case without the cylinder. 


\section{Bibliography}

[1] Theodor Schwenk. Sensitive Chaos: The Creation of Flowing Forms in Water and Air. Rudolf Steiner Press, second edition, 1990.

[2] Julio M. Ottino. The art of mixing with an admixture of art: fluids, solids, and visual imagination. Phys. Fluids, 22, 021301, 2010.

[3] A. Roshko. Perspectives on bluff body aerodynamics. J. Wind Eng. and Industrial Aerodyn., 49:79-100, 1993.

[4] D. Hasegawa. How islands stir and fertilize the upper ocean. Geophysical Research Letters, $31,2004$.

[5] M. S. Triantafyllou, G. S. Triantafyllou, and D. K. P. Yue. Hydrodynamics of fishlike swimming. Annu. Rev. Fluid Mech., 32:33-53, 2000.

[6] István Scheuring, György Károlyi, Áron Péntek, Tamás Téls, and Zoltán Torczkai. A model for resolving the plankton paradox: coeistence in open flows. Freshwater Biology, 45:123-132, 2000 .

[7] B. L. Jensen, B. M. Sumer, H. R. Jensen, and J. Fredsœ. Flow around and forces on a pipeline near a scoured bed in steady current. ASME J. Offshore Mech. and Arctic Engrg., 112:206-213, 1990.

[8] J. M. Kim and N. M. Komerath. Summary of the interaction of a rotor wake with a circular cylinder. AIAA Journal, 33(3):470-478, 1995.

[9] P. W. Bearman. Near wake flows behind two- and three-dimensional bluff bodies. J. Wind Eng. and Industrial Aerodyn., 69-71:33-54, 1997.

[10] Albert Lee and Eric Loth. 3-D droplet dispersion in a cylinder wake flow. Technical report, Tampa, FL, 2010. 7th International Conference on Multiphase Flow.

[11] D. J. Brandon and S. K. Aggarwal. A numerical investigation of particle decomposition of a square cylinder placed in a channel flow. Aerosol Sci. Technol., 34(4):340-352, 2001.

[12] K. Furukawa and E. Wolanski. Shallow-water frictional effects in island wakes. Estuarine, Coastal and Shelf Science, 46:599-608, 1998.

[13] Karen J. Heywood, Eric D. Barton, and John H. Simpson. The effects of flow disturbance by an oceanic island. J. Marine Res., 48:55-73, 1990. 
[14] Changming Dong and James C. McWilliams. A numerical study of island wakes in the Southern California Bight. Continental Shelf Research, 27:1233-1248, 2007.

[15] Kelvin J. Richards and Stuart J. Brentnall. The impact of diffusion and stirring on the dynamics of interacting populations. J. Theor. Biol., 238:340-347, 2006.

[16] Editor Gisela E. Speidel. Stirring and mixing of marine ecosystems. IPRC Climate Newsletter vol. 3, no. 2, International Pacific Research Center, January 2004. Hagadone Printing Co.

[17] György Károlyi, Áron Péntek, István Scheuring, Tamás Téls, and Zoltán Torczkai. Chaotic flow; the physics of species coexistence. Proc. Nat'l. Acad. Sci. USA, 97(25):13661-13665, 2000 .

[18] K. J. Richards. Physical processes in the benthic boundary layer. Phil. Trans. R. Soc. Lond. A, 331:3-13, 1990.

[19] K. Miller and C. Mundy. Rapid settlement in broadcast spawning corals: implications for larval dispersal. Coral Reefs, 22:99-106, 2003.

[20] J. B. C. Jackson. Modes of dispersal of clonal benthic invertebrates: consequences for species' distributions and genetic structure of local populations. Bulletin of Marine Science, 39(2):588606, 1986.

[21] John P. Crimaldi, Jillian R. Cadwell, and Jeffrey B. Weiss. Reaction enhancement of isolated scalars by vortex stirring. Phys. Fluids, 20, 073605, 2008.

[22] B. M. Sumer and J. Fredsoe. Hydrodynamics Around Cylindrical Structures. World Scientific Publishing Co., 1997.

[23] C. H. K. Williamson. Vortex dynamics in the cylinder wake. Annu. Rev. Fluid Mech., 28:477539, 1996.

[24] J. H. Gerrard. The mechanics of the formation region of vortices behind bluff bodies. J. Fluid Mech., 25:401-413, 1966.

[25] Owen M. Griffin. A note on bluff body vortex formation. J. Fluid Mech., 284:217-224, 1995.

[26] R. B. Green and J. H. Gerrard. Vorticity in the near wake of a circular cylinder at low Reynolds numbers. J. Fluid Mech., 246:675-691, 1993.

[27] M. Susan Bloor. The transition to turbulence in the wake of a circular cylinder. J. Fluid Mech., 19(2):290-304, 1964.

[28] J. H. Gerrard. The wakes of cylindrical bluff bodies at low Reynolds number. Phil. Trans. R. Soc. Lond. A, 288(1354):351-382, 1978.

[29] V. Babu and Arunn Narasimhan. Investigation of vortex shedding behind a porous square cylinder using lattice Boltzmann method. Phys. Fluids, 22, 053605, 2010.

[30] E. Achenbach ad E. Heinecke. On vortex shedding from smooth and rough cylinders in the range of Reynolds numbers. J. Fluid Mech., 109:239-251, 1981. 
[31] Pranesh Muralidhar, Nangelie Ferrer, Robert Daniello, and Jonathan P. Rothstein. Influence of slip on the flow past superhydrophobic circular cylinders. J. Fluid Mech., 680:459-476, 2011.

[32] Dong-Hyeog Yoon, Kyung-Soo Yang, and Choon-Bum Choi. Flow past a square cylinder with an angle of incidence. Phys. Fluids, 22, 043603, 2010.

[33] C. P. Jackson. A finite-element study of the onset of vortex shedding in flow past variously shaped bodies. J. Fluid Mech., 182:23-45, 1987.

[34] Jacob H. Masliyah and Norman Epstein. Steady symmetric flow past elliptical cylinders. Ind. Eng. Chem. Fundam., 10(2):293-299, 1971.

[35] C. M. Sewatkar, Atul Sharma, and Amit Agrawal. On the effect of Reynolds number for flow around a row of square cylinders. Phys. Fluids, 21, 083602, 2010.

[36] S. J. Xu, Y. Zhou, and R. M. C. So. Reynolds number effects on the flow structure behind two side-by-side cylinders. Phys. Fluids, 15(5):1214-1219, 2003.

[37] M. Gaster. Vortex shedding from circular cylinders at low Reynolds numbers. J. Fluid Mech., 46(4):749-756, 1971.

[38] K. Lam and Y. F. Lin. Effects of wavelength and amplitude of a wavy cylinder in a cross-flow at low Reynolds numbers. J. Fluid Mech., 620:195-220, 2009.

[39] Warren Dunn and Stavros Tavoularis. Vortex shedding from a step-cylinder in spanwise sheared flow. Phys. Fluids, 23, 035109, 2011.

[40] Mohamed Sukri Mat Ali. Low Reynolds number flow over a square cylinder with a splitter plate. Phys. Fluids, 23, 033602, 2011.

[41] J. Sheridan, J. C. Lin, and D Rockwell. Flow past a cylinder close to a free surface. J. Fluid Mech., 330:1-30, 1997.

[42] Masaru Kiya, Hisataka Tamura, and Mikio Arie. Vortex shedding from a circular cylinder in moderate-Reynolds-number shear flow. J. Fluid Mech., 141(4):721-735, 1980.

[43] H. G. C. Woo, J. E. Cermak, and J. A. Peterka. Secondary flows and vortex formation around a circular cylinder in constant-shear flow. J. Fluid Mech., 204:523-542, 1989.

[44] Madeleine Coutanceau and Roger Bouard. Experimental determination of the main features of a viscous flow in the wake of a circular cylinder in uniform translation. part 2. unsteady flow. J. Fluid Mech., 79(part 2):257-272, 1977.

[45] D. J. Tritton. Experiments on the flow past a circular cylinder at low Reyolds numbers. J. Fluid Mech., 6(04):547-567, 1959.

[46] M. Hammache and M. Gharib. An experimental study of the parallel and oblique vortex shedding from circular cyinders. J. Fluid Mech., 232:567-590, 1991.

[47] Charles A. Kossack. Steady simple shear flow past a circular cylinder at moderate Reynolds numbers: a numerical solution. J. Fluid Mech., 66:353-376, 1974. 
[48] M. M. Zdravkovich. Flow Around Circular Cylinders Volume 1: Fundamentals. Oxford University Press, USA, 1997.

[49] E. Berger and R. Willie. Periodic flow phenomena. Annu. Rev. Fluid Mech., 4:313-340, 1971.

[50] Bernd R. Noack, Konstantin Afanasiev, Marek Morzyński, Gilead Tadmor, and Frank Thiele. A hierarchy of low-dimensional models for the transient and post-transient cylinder wake. J. Fluid Mech., 497:335-363, 2003.

[51] Carl Eckart. An analysis of the stirring and mixing processes in incompressible fluids. J. Marine Res., 7(3):265-275, 1948.

[52] G. K. Batchelor. An Introduction to Fluid Dynamics. Oxford University Press, 1967.

[53] James P. Gleeson. Transient micromixing: Examples of laminar and chaotic stirring. Phys. Fluids, 17, 100614, 2005.

[54] Jean-Luc Thiffeault. Lecture 1: Stirring and mixing. Technical report, Woods Hole Oceanographic Institution, Woods Hole, Ma, June 2010.

[55] J. M. Ottino. The kinematics of mixing: stretching, chaos, and transport. Cambridge University Press, 1989.

[56] Jean-Luc Thiffeault. A bound on mixing efficiency for the advection-diffusion equation. J. Fluid Mech., 521:105-114, 2004.

[57] J. M. Ottino. Mixing, chaotic advection, and turbulence. Annu. Rev. Fluid Mech., 22:207-253, 1990.

[58] Meunier2003. How vortices mix. J. Fluid Mech., 476:213-222, 2003.

[59] M. R. Mackley and R. M. C. Neves Saraiva. The quantitayive description of fluid mixing using Lagrangian- and concentration-based numerical approaches. Chem. Engr. Sci., 54:159-170, 1999.

[60] P. Flohr and J. C. Vassilicos. Accelerated scalar dissipation in a vortex. J. Fluid Mech., 248:295-317, 1997.

[61] John P. Crimaldi, Jillian R. Hartford, and Jeffery B. Weiss. Reaction enhancement of point sources due to vortex stirring. Phys. Rev. E, 74, 2006.

[62] Baki M. Cetegen and Nazri Mohamad. Experiments on liquid mixing and reaction in a vortex. J. Fluid Mech., 249:391-414, 1993.

[63] Konrad Bajer, Andrew P. Bassom, and Andrew D. Gilbert. Accelerated diffusion in the centre of a vortex. J. Fluid Mech., 437:395-411, 2001.

[64] Scott A. Socolofsky and Gerhard H. Jirka. Special topics in mixing and transport processes in the environment. Texas A\&M University, 2005. Fifth edition.

[65] M. Behr, H. Hastreiter, S. Mittal, and T. E. Tezduyar. Incompressible flow past a circular cylinder: dependence of the computed flow field on the location of the lateral boundaries. Comput. Methods Appl. Mech. Engrg., 123:309-316, 1995. 
[66] Anatol Roshko. On the drag and shedding frequency of two-dimensional bluff bodies. Technical Note 3169, National Advisory Committee for Aeronautics, July 1954.

[67] C. H. K. Williamson. Oblique and parallel modes of vortex shedding in the wake of a circular cylinder at low Reynolds numbers. J. Fluid Mech., 206:579-627, 1989. 
Appendix A

Model Parameters 
Table A.1: Model Variables

\begin{tabular}{|c|c|c|c|}
\hline Name & Expression & Value & Description \\
\hline rho & $998.2\left[\mathrm{~kg} / \mathrm{m}^{3}\right]$ & $998.2\left[\mathrm{~kg} / \mathrm{m}^{3}\right]$ & Density \\
\hline eta & $1.002 \times 10^{-3}[\mathrm{~Pa} \mathrm{~s}]$ & $0.001002[\mathrm{~kg} /(\mathrm{m} \mathrm{s})]$ & Dynamic viscosity \\
\hline $\mathrm{D}$ & $(\mathrm{eta} / \mathrm{rho}) / \mathrm{Sc}$ & $1.003807 \times 10^{-7}\left[\mathrm{~m}^{2} / \mathrm{s}\right]$ & Diffusivity \\
\hline h_cyl & $11[\mathrm{~cm}]$ & $0.11[\mathrm{~m}]$ & Dist from bottom bdry to cyl bdry \\
\hline h_in & $22[\mathrm{~cm}]$ & $0.22[\mathrm{~m}]$ & Total inlet height \\
\hline ds & ds_star $\times$ diam & {$[\mathrm{m}]$} & Dimensional scalar separation \\
\hline $\mathrm{df}$ & df_star $\times$ diam & {$[\mathrm{m}]$} & Filament width \\
\hline u_in & $0.77[\mathrm{~cm} / \mathrm{s}]$ & $0.0077[\mathrm{~m} / \mathrm{s}]$ & velocity \\
\hline x_flight & $0.01795[\mathrm{~m}]$ & $0.01795[\mathrm{~m}]$ & Dist from Mike's release loc to Tanaya's \\
\hline $\mathrm{c} 0$ & $1\left[\mathrm{~mol} / \mathrm{m}^{3}\right]$ & $1\left[\mathrm{~mol} / \mathrm{m}^{3}\right]$ & Input concentration scale \\
\hline $\mathrm{k}$ & $(\mathrm{Da} \times \mathrm{D}) /\left(\mathrm{c} 0 \times \mathrm{diam}^{2}\right)$ & $6.223615 \times 10^{-4}\left[3^{3} / \mathrm{mol} \mathrm{s}\right]$ & Reaction rate constant \\
\hline diam & $0.0127[\mathrm{~m}]$ & $0.0127[\mathrm{~m}]$ & Cylinder diameter \\
\hline St & 0.179 & 0.179 & Strouhal number \\
\hline T_s & $\operatorname{diam} /(\mathrm{St} \times$ u_in $)$ & $9.214249[\mathrm{~s}]$ & Shedding period \\
\hline $\operatorname{Re}$ & u_in $\times \operatorname{diam} /($ eta $/$ rho $)$ & 97.41914 & Reynolds number \\
\hline $\mathrm{Pe}$ & diam $\times$ u_in $/ D$ & 974.191397 & Peclet number \\
\hline $\mathrm{Sc}$ & 10 & 10 & Schmidt number \\
\hline $\mathrm{Da}$ & 0.01 & 0.01 & Damkohler number \\
\hline ds_star & 0.25 & 0.25 & Nondimensional scalar separation \\
\hline df_star & 3 & 3 & Nondimensional filament width \\
\hline
\end{tabular}


Table A.2: Free mesh parameters

\begin{tabular}{cc} 
Mesh Parameter & Value \\
\hline Maximum element scaling factor & 1 \\
Element growth rate & 1.2 \\
Mesh curvature factor & 0.05 \\
Mesh curvature cutoff & 0.1 \\
Resolution of narrow regions & 1
\end{tabular}


Appendix B

MATLAB Code 


\section{B.1 Numerical Integration Solution (Eqn. 2.21)}

$\%$ Constants

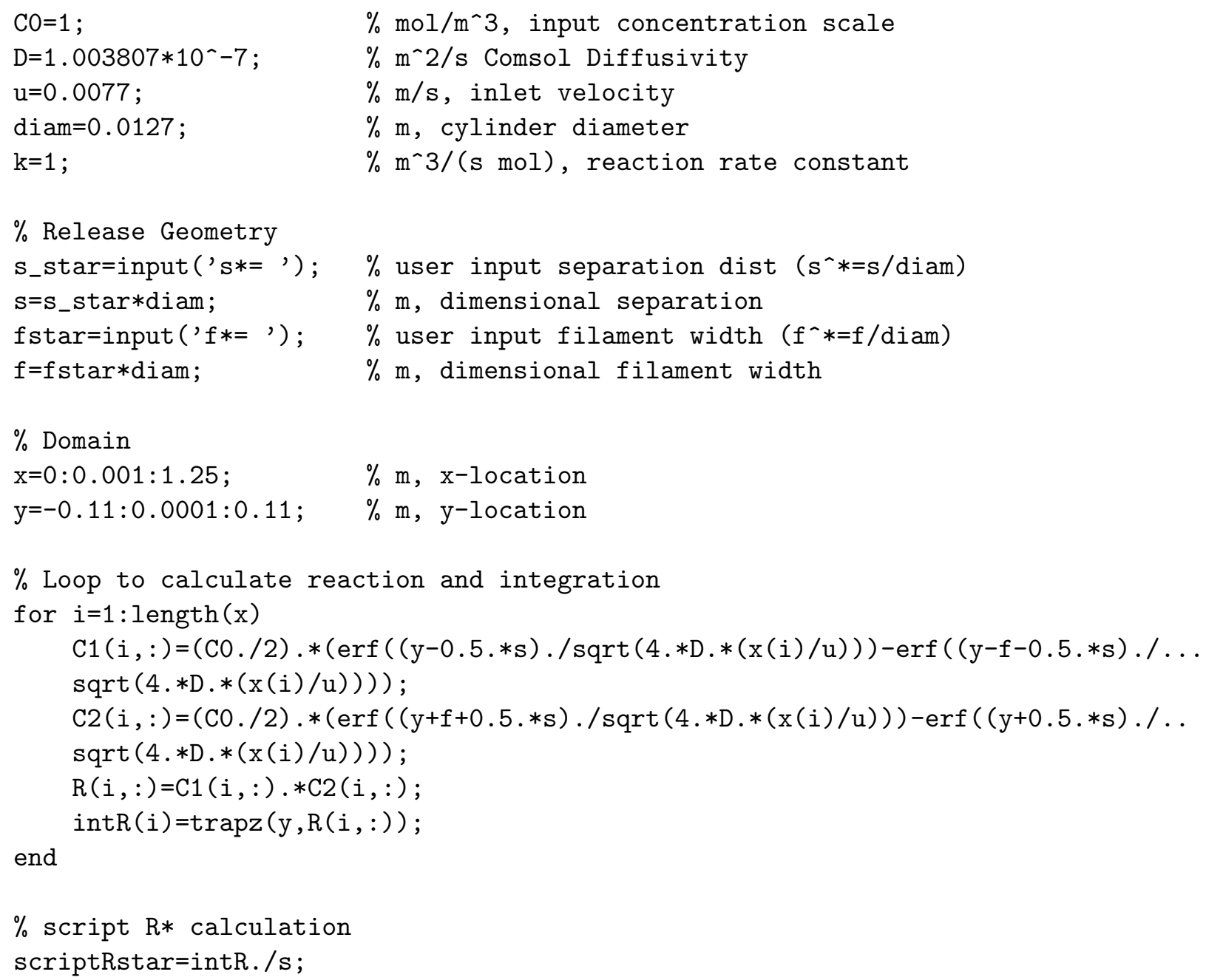

\section{B.2 Analytical Solution (Eqn. 4.6)}

$\%$ Constants and Parameters

$\mathrm{x}=0: 0.01: 3 ; \quad \% \mathrm{~m}$, distance

diam $=0.0127 ; \quad \% \mathrm{~m}$, cylinder diameter

$\mathrm{u}=0.0077 ; \quad \% \mathrm{~m} / \mathrm{s}$, velocity

$\mathrm{s}=0.01 ; \quad \% \mathrm{~m}$, arbitrary separation

$\mathrm{D}=1.003807 \mathrm{E}-7 ; \quad \% \mathrm{~m}^{\wedge} 2 / \mathrm{s}$ diffusivity

$\%$ lambda calculation 
$\mathrm{xstar}=\mathrm{x} / \mathrm{s}$;

$\mathrm{Pe}=(\mathrm{u} * \mathrm{~s}) /(16 * \mathrm{D})$;

lambda $=\operatorname{sqrt}($ xstar. $/ \mathrm{Pe})$;

$\%$ script $\mathrm{R} *$ calculation

ScriptR $=($ lambda. $/ 2) . *\left((1 . / \operatorname{sqrt}(2 . *\right.$ pi $)) . * \exp \left(-2 . /\right.$ lambda. $\left.{ }^{\wedge} 2\right)-(1 . /$ lambda $) . * \ldots$ $\operatorname{erf}(-\operatorname{sqrt}(2) . /$ lambda $)-(1 . /$ lambda $))$;

\section{B.3 Laterally integrated, phased averaged reaction rate processed from data exported from COMSOL}

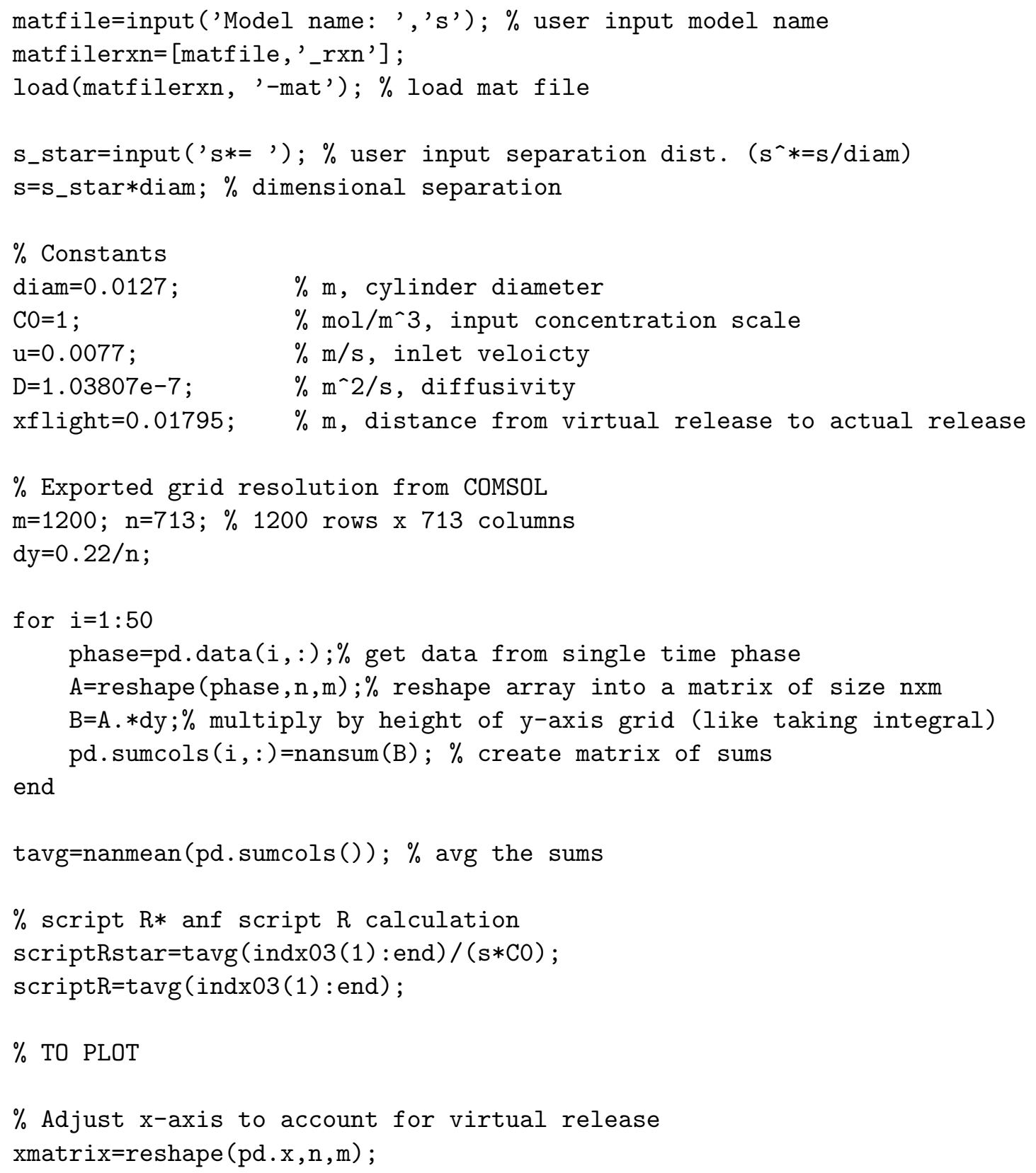


xcoordsAct=xmatrix (end,: ); \%actual model dimensions

indx03 $=$ find ( $x$ coordsAct $>-0.03)$; \% index of virtual release location

xcoordsShort=xcoordsAct (indx03(1):end); \%truncate everything before actual release xcoordsShift=xcoordsShort $+0.03+x f l i g h t ; \%$ shift $x c o o r d s$ to start at virtual release $\mathrm{xcoords}=\mathrm{xcoords}$ Shift;

$\mathrm{NDx}=\mathrm{xcoords}$./diam;

$\%$ lambda calculation

$\mathrm{xstar}=\mathrm{xcoords} / \mathrm{s} ; \mathrm{dy}=0.22 / \mathrm{n}$;

$\mathrm{Pe}=(\mathrm{u} * \mathrm{~s}) /(16 * \mathrm{D})$;

lambda $=$ sqrt $($ xstar $/ \mathrm{Pe})$;

$\%$ plot script $\mathrm{R}$ vs lambda

plot (lambda, scriptR, 'k-- ', 'LineWidth', 2);

$\mathrm{xlabel}(' \$ \backslash$ lambda $=\backslash \operatorname{sqrt}\{\mathrm{x} /$ textrm $\{\mathrm{Pe}\}\}$ ' , 'Interpreter', '’latex', 'fontsize',18)

ylabel ('\$ $\$$ mathcal $\{\mathrm{R}\} \$$ ', 'Interpreter' , 'latex', 'fontsize', 18, 'rotation', 0)

set (gca, 'FontSize', 18, 'fontname', 'times new roman') 
Appendix C

Proof for integral identity Eqn. 4.5 
We have the following

$$
\int_{-\infty}^{\infty}[1+\operatorname{erf}(\xi+\alpha)][1+\operatorname{erf}(-\xi+\alpha)] \mathrm{d} \xi
$$

Since for $x \gg 1$,

$$
\operatorname{erf}(x)=1-\frac{2}{\sqrt{\pi}} \int_{x}^{\infty} \exp \left(-u^{2}\right) \mathrm{d} u
$$

Then Eqn. C.1 is equivalent to

$$
\int_{-\infty}^{\infty}[1+\operatorname{erf}(\xi+\alpha)][1+\operatorname{erf}(-\xi+\alpha)] \mathrm{d} \xi=\frac{4}{\pi} \int_{-\infty}^{\infty} \int_{-\infty}^{\alpha+\xi} \int_{-\infty}^{\alpha-\xi} \exp \left(-s^{2}-t^{2}\right) \mathrm{d} s \mathrm{~d} t \mathrm{~d} \xi
$$

Note that $s \leq \alpha+\xi$ and $t \leq \alpha-\xi$; i.e. $s-\alpha \leq \xi \leq \alpha-t$ and $s+t \leq 2 \alpha$. Thus,

$$
\begin{aligned}
\frac{4}{\pi} \int_{-\infty}^{\infty} \int_{-\infty}^{\alpha+\xi} \int_{-\infty}^{\alpha-\xi} \exp \left(-s^{2}-t^{2}\right) \mathrm{d} s \mathrm{~d} t \mathrm{~d} \xi & =\frac{4}{\pi} \iint_{s+t \leq 2 a} \int_{s-a}^{a-t} \exp \left(-s^{2}-t^{2}\right) \mathrm{d} \xi \mathrm{d} s \mathrm{~d} t \\
& =\frac{4}{\pi} \int_{-\infty}^{\infty} \int_{-\infty}^{\infty}(2 \alpha-s-t)+\exp \left(-s^{2}-t^{2}\right) \mathrm{d} s \mathrm{~d} t
\end{aligned}
$$

Change variables: $u=(s+t) / \sqrt{2}$ and $v=(s-t) / \sqrt{2}$ so that $s=(u+v) / \sqrt{2}$ and $t=(u-v) / \sqrt{2}$ :

$$
\begin{aligned}
\frac{4}{\pi} \int_{-\infty}^{\infty} \int_{-\infty}^{\infty}(2 \alpha-s-t)+\exp \left(-s^{2}-t^{2}\right) \mathrm{d} s \mathrm{~d} t & =\frac{4}{\pi} \int_{-\infty}^{\infty} \int_{-\infty}^{\infty}(2 \alpha-\sqrt{2} u)+\exp \left(-u^{2}-v^{2}\right) \mathrm{d} u \mathrm{~d} v \\
& =\frac{4}{\sqrt{\pi}} \int_{-\infty}^{\sqrt{2} \alpha}(2 \alpha-\sqrt{2} u) \exp \left(-u^{2}\right) \mathrm{d} u \\
& =4 a(\operatorname{erf}(\sqrt{2} \alpha)+1)-\frac{4}{\sqrt{\pi}} \int_{-\infty}^{\sqrt{2} \alpha} \sqrt{2} u \exp \left(-u^{2}\right) \mathrm{d} u \\
& =4 a(\operatorname{erf}(\sqrt{2} \alpha)+1)-\frac{2 \sqrt{2}}{\sqrt{\pi}} \int_{-\infty}^{\sqrt{2} \alpha} \exp \left(-u^{2}\right) \mathrm{d} u^{2} \\
& =4 a(\operatorname{erf}(\sqrt{2} \alpha)+1)-\frac{2 \sqrt{2}}{\sqrt{\pi}} \exp \left(-2 \alpha^{2}\right) \\
& =4 \alpha \operatorname{erf}(\sqrt{2} \alpha)+4 \alpha-\frac{2 \sqrt{2}}{\sqrt{\pi}} \exp \left(-2 \alpha^{2}\right)
\end{aligned}
$$

Therefore,

$$
\int_{-\infty}^{\infty}[1+\operatorname{erf}(\xi+\alpha)][1+\operatorname{erf}(-\xi+\alpha)] \mathrm{d} \xi=4\left[\frac{1}{\sqrt{2 \pi}} \exp \left(-2 \alpha^{2}\right)+\alpha \operatorname{erf}(\sqrt{2} \alpha)+\alpha\right]
$$

